Aus der Poliklinik für Präventive Zahnmedizin,

Parodontologie und Kariologie

(Prof. Dr. med. dent. A. Wiegand)

im Zentrum Zahn-, Mund- und Kieferheilkunde

der Medizinischen Fakultät der Universität Göttingen

\title{
Prävalenz von und Risikofaktoren für dentale Erosionen bei Göttinger Kindergartenkindern
}

\author{
INAUGURAL-DISSERTATION \\ zur Erlangung des Doktorgrades \\ für Zahnheilkunde \\ der Medizinischen Fakultät der \\ Georg-August-Universität zu Göttingen
}

vorgelegt von

Christina Müller-Pflanz (geb. Pflanz)

aus

Fulda

Göttingen 2017 
Dekan:

Referent/in:

Ko-Referent/in:

Drittreferent/in:
Prof. Dr. rer. nat. H. K. Kroemer

Prof. Dr. med. dent. A. Wiegand

Prof. Dr. med. K. Brockmann

Datum der mündlichen Prüfung: 20.03.2018 
Hiermit erkläre ich, die Dissertation mit dem Titel „Prävalenz von und Risikofaktoren für dentale Erosionen bei Göttinger Kindergartenkindern" eigenständig angefertigt und keine anderen als die von mir angegebenen Quellen und Hilfsmittel verwendet zu haben.

Göttingen, den 


\section{Inhaltsverzeichnis}

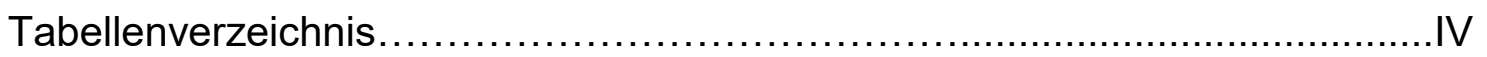

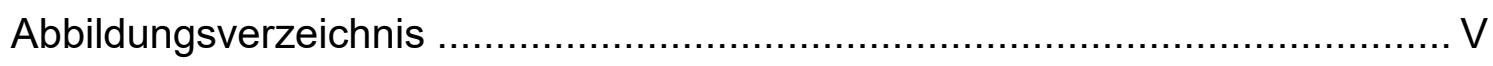

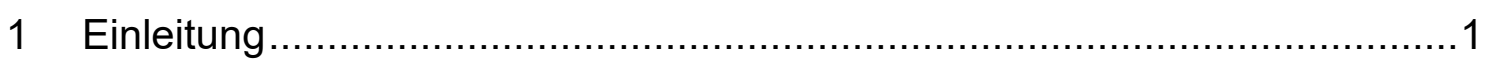

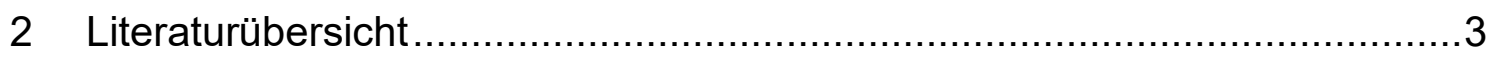

2.1 Definition und klinisches Erscheinungsbild von dentalen Erosionen ......3

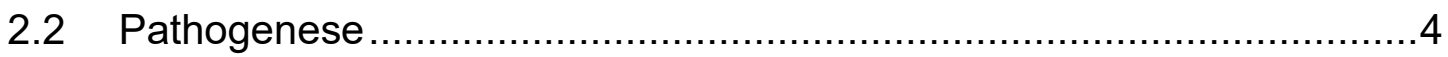

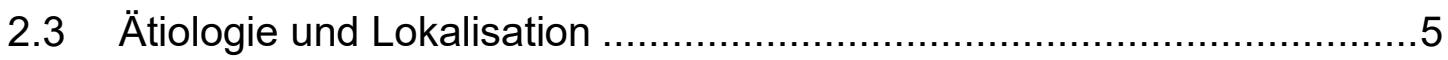

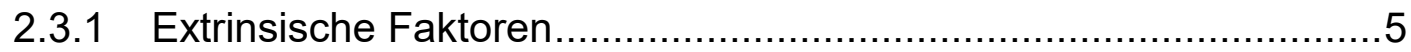

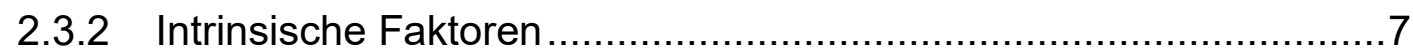

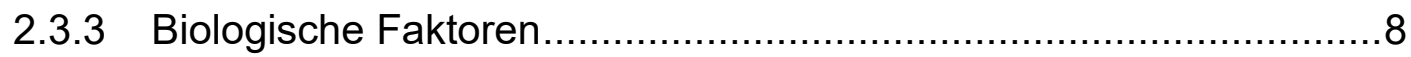

2.3.3.1 Die Interaktion von Erosion, Attrition und Abrasion ................. 10

2.3.3.2 Besonderheiten im Milchgebiss ................................... 10

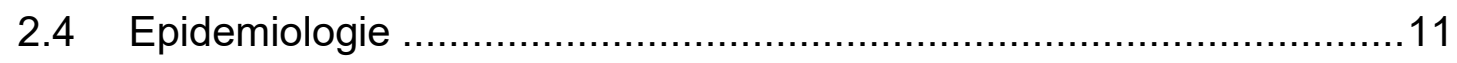

2.4.1 Erosionsindizes für epidemiologische Studien ..........................11

2.4.2 Prävalenz bei Erwachsenen, Jugendlichen und Kindern ............... 14

2.4.3 Inzidenz und Progression................................................. 18

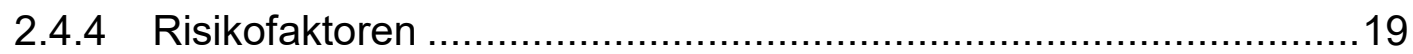

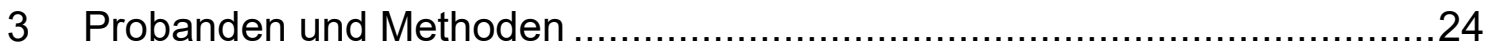

3.1 Kontaktaufnahme und Probandengut...........................................24

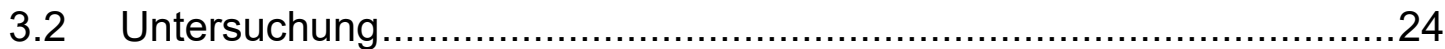

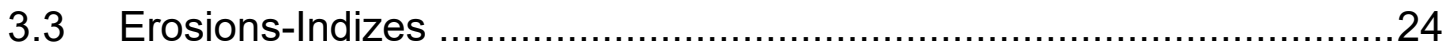

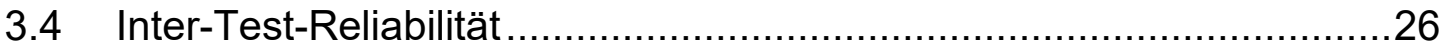

3.5 Inter- und Intra-Untersucher-Reliabilität ...................................28

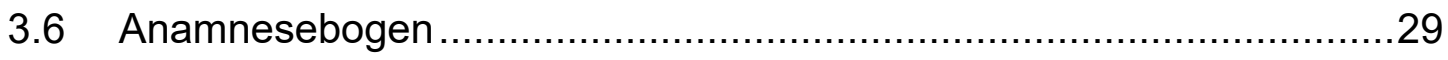

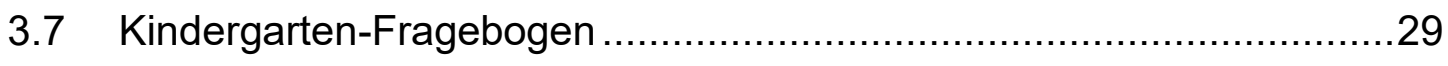

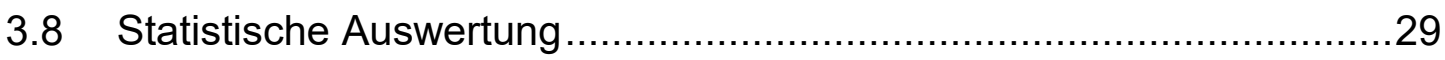


4 Ergebnisse

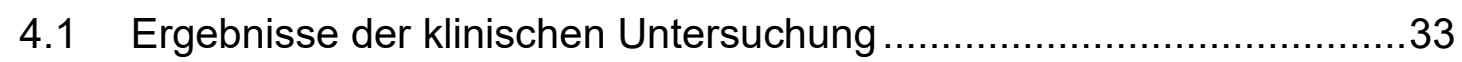

4.1.1 Prävalenz der Erosionen in Abhängigkeit von Alter und Geschlecht

4.1.2 Prävalenz der Erosionen in Ober- und Unterkiefer und Frontund Seitenzahnbereich

4.1.3 Schweregrad und Lokalisation der Erosionen anhand des O'Sullivan-Index

4.1.4 Schweregrad und Lokalisation der Erosionen anhand des BEWE-Index.

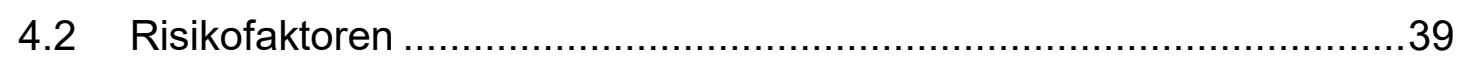

4.2.1 Rolle der Kindergärten ......................................................... 40

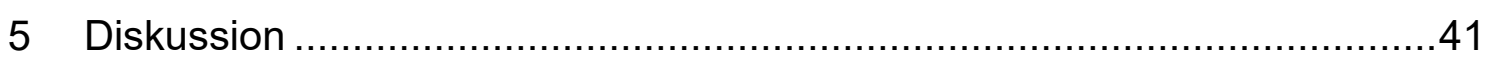

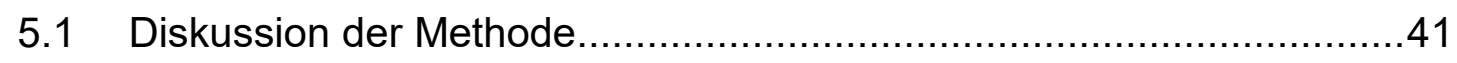

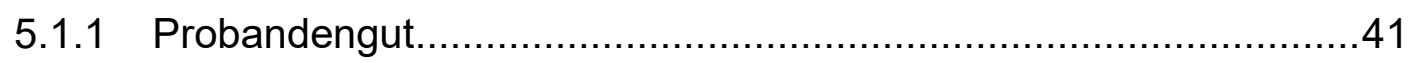

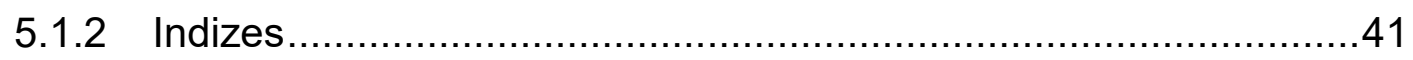

5.1.3 Klinische Untersuchung …..................................................

5.1.4 Anamnesebogen und Kindergarten-Fragebogen .........................42

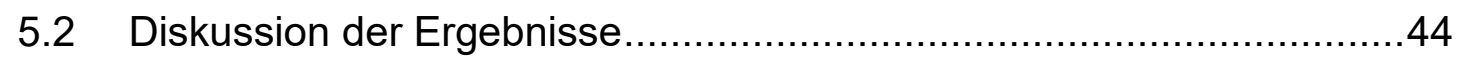

5.2.1 Ergebnisse der klinischen Untersuchung ..................................4

5.2.1.1 Prävalenz und Schwere der Erosionen ...................................44

5.2.1.2 Prävalenz und Schwere der Erosionen in Abhängigkeit

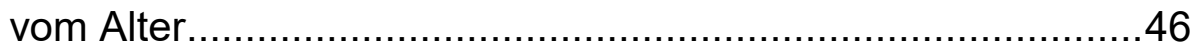

5.2.1.3 Prävalenz und Schwere der Erosionen in Abhängigkeit

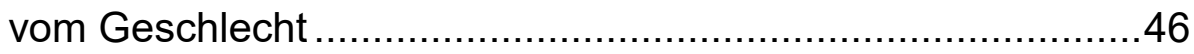

5.2.1.4 Prävalenz der Erosionen in Ober- und Unterkiefer und Front- und Seitenzahnbereich.............................................. 47

5.2.1.5 Lokalisation der erosiven Läsionen auf den Zahnflächen ........48

5.2.2 Anamnestische Risikofaktoren.... 


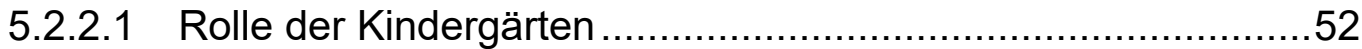

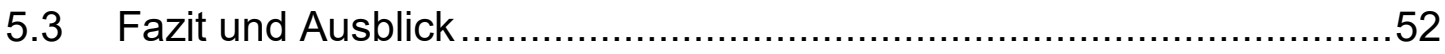

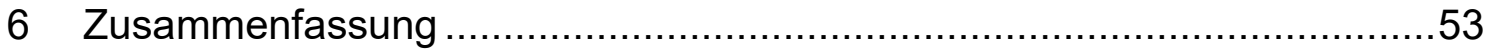

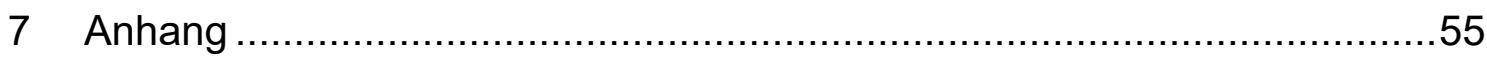

7.1 Anamnesebogen und Kindergarten-Fragebogen ...............................55

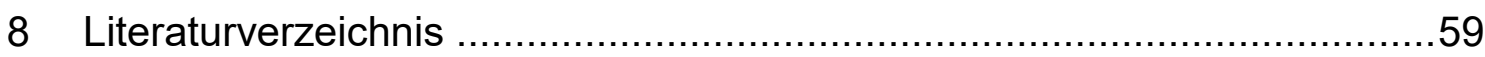




\section{Tabellenverzeichnis}

Tabelle 2.1: Erosions-Index nach Eccles 1979.......................................12

Tabelle 2.2: „Tooth Wear Index“ nach Smith und Knight 1984......................13

Tabelle 2.3: Prävalenz dentaler Erosionen bei Erwachsenen in chronologischer Reihenfolge................................................16

Tabelle 2.4: Prävalenz dentaler Erosionen bei Kindern und Jugendlichen zwischen 10 und 17 Jahren in chronologischer Reihenfolge.

Tabelle 2.5: Prävalenz dentaler Erosionen bei Kindern bis einschließlich 9 Jahre in chronologischer Reihenfolge.

Tabelle 2.6: Epidemiologische Studien bei Kindern zwischen 2 und 9 Jahren, die eine Risikofaktoranalyse enthalten (in chronologischer Reihenfolge).

Tabelle 3.1: Index zur Klassifizierung dentaler Erosionen im Milchgebiss

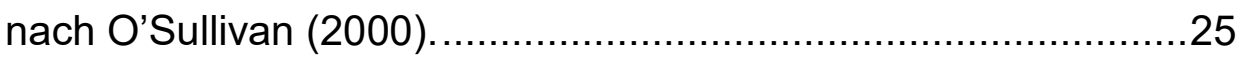

Tabelle 3.2: BEWE-Bewertungssystem nach Bartlett et al. (2008)................26

Tabelle 3.3: Schema zur Überführung der O'Sullivan-Codierung in die

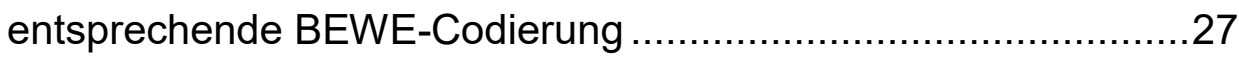

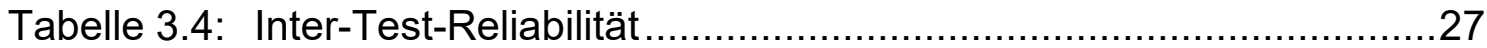

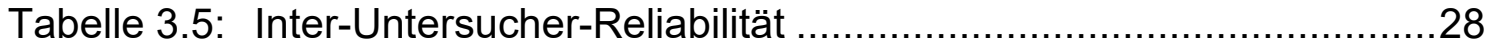

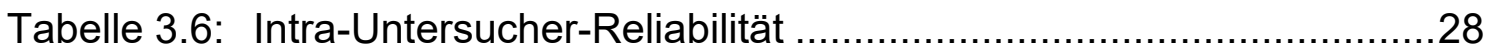

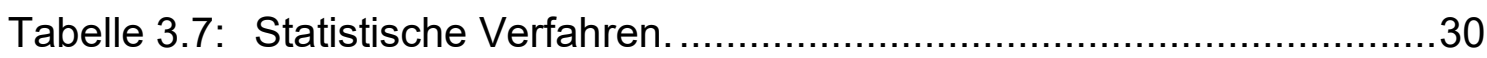

Tabelle 3.8: Risikofaktoren: Messgrößen und statistische Verfahren. .............30

Tabelle 3.9: Potentielle Risikofaktoren, die als Regressoren verwendet

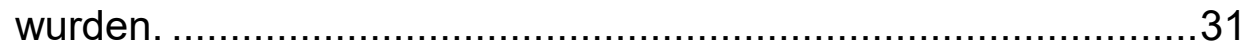

Tabelle 4.1: Prävalenz der Erosionen nach Altersgruppen.............................34

Tabelle 4.2: Anteil der Kinder mit Erosionen im Ober- und Unterkiefer je

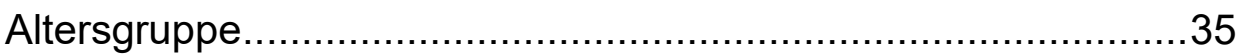

Tabelle 4.3: Betroffene Flächen von Front- und Eckzähnen...........................36

Tabelle 4.4: Betroffene Flächen von Molaren. ................................................ 37

Tabelle 4.5: Verteilung der untersuchten Kinder entsprechend der RisikoKlassifizierung nach dem BEWE-Summenscore. 39

Tabelle 5.1: In verschiedenen Studien festgestellte Prävalenzen in den entsprechenden Altersklassen. 


\section{Abbildungsverzeichnis}

Abbildung 4.1: Übersichtskarte und Verteilungsmuster der Kindergärten, die an der vorliegenden Studie teilgenommen haben.

Abbildung 4.2: Prävalenz dentaler Erosionen bei allen Zähnen des Milchgebisses.

Abbildung 4.3: Verteilung der Erosionen entsprechend dem BEWE-Index bei allen Zähnen des Milchgebisses.

Abbildung 4.4: Verteilung der Erosionen in Ober- und Unterkiefer bei 3Jährigen und 4-Jährigen.

Abbildung 4.5: Verteilung der Erosionen in Ober- und Unterkiefer bei 5Jährigen und 6-7-Jährigen. 38

Abbildung 7.1: Anamnesebogen, von den Erziehungsberechtigten auszufüllen: Seite 1.

Abbildung 7.2: Anamnesebogen, von den Erziehungsberechtigten auszufüllen: Seite 2 .

Abbildung 7.3: Fragebogen, von den Kindergärten auszufüllen: Seite 1.......57

Abbildung 7.4: Fragebogen, von den Kindergärten auszufüllen: Seite 2. 58 


\section{Einleitung}

Erosionen werden als die chemische und irreversible Auflösung der Zahnhartsubstanzen durch Säuren nichtbakteriellen Ursprungs definiert. Der Prozess der Erosion zeichnet sich durch eine schichtweise Abtragung der Zahnhartsubstanzen aus. Patienten mit schweren Erosionen leiden unter Hypersensibilitäten und ästhetischen Einschränkungen. Darüber hinaus können Erosionen die Funktionalität des Gebisses beeinträchtigen und im fortlaufenden Prozess bis zum vollständigen Verlust der okklusalen Morphologie und der Anatomie des Zahnes führen (Ganss und Lussi 2014, Lussi et al. 2011). Sobald der erste Milchzahn in die Mundhöhle eruptiert, ist er einem möglichen Risiko ausgesetzt, Erosionen zu entwickeln. Da die Milchzähne kleiner als ihre bleibenden Nachfolger sind und ihre Schmelzschicht dünner ist, können Milchzähne verhältnismäßig schneller und schwerer erodieren als bleibende Zähne (Hunter et al. 2000). Es wird zudem angenommen, dass das Auftreten von dentalen Erosionen im Milchgebiss zu einem gehäuften Auftreten von Erosionen in der bleibenden Dentition führt (Ganss et al. 2001).

Zur Prävalenz und Ätiologie von Erosionen wurden zahlreiche Studien bei Kindern und Erwachsenen aller Altersklassen durchgeführt. Die Prävalenz für Kinder im Milchgebiss wird in verschiedenen Studien mit 0,6\% (Moimaz et al. 2013) bis $100 \%$ (Jaeggi und Lussi 2004) angegeben. In Deutschland wurde bisher nur eine Untersuchung bei Kindern zwischen 2 und 7 Jahren durchgeführt (Wiegand et al. 2006). International zeigt sich eine große Varianz der Studienergebnisse. Sie wird auf grundlegende Länderunterschiede und eine uneinheitliche Methodik der Untersuchungen zurückgeführt. Es gibt jedoch Hinweise darauf, dass die Prävalenz von Erosionen bei Kindern in den letzten Jahren insgesamt zugenommen hat (Nunn et al. 2003, Jaeggi und Lussi 2014).

Als potentielle Risikofaktoren für dentale Erosionen bei Kindern werden in der Fachliteratur vor allem der regelmäßige Konsum von Softdrinks, Fruchtsäften und sauren Nahrungsmitteln, aber auch häufiges Erbrechen, die RefluxKrankheit und die Verwendung eines Asthma-Sprays diskutiert. Der hauptursächliche Risikofaktor scheint aber eine säurehaltige Ernährung zu sein. Salas et al. (2015) zeigen in einer aktuellen Meta-Analyse, dass besonders ein hoher 
Konsum von Softdrinks mit dem Risiko einhergeht, Erosionen zu entwickeln. Eltern und Kinder sollten frühzeitig über die Entstehung und die Folgen von Erosionen aufgeklärt werden, um das Auftreten solcher Zahnhartsubstanzdefekte schon im Vorfeld erfolgreich zu verhindern. Eine genaue Kenntnis der Einfluss- und Risikofaktoren für Erosionen im Milchgebiss ist dafür unerlässlich.

Die Ziele der vorliegenden Studie waren deshalb, die Prävalenz, den Schweregrad und die Lokalisation von dentalen Erosionen bei Göttinger Kindergartenkindern zwischen 3 und 7 Jahren zu erheben und potentielle Risikofaktoren zu ermitteln. 


\section{Literaturübersicht}

\subsection{Definition und klinisches Erscheinungsbild von dentalen Erosionen}

Bei dentalen Erosionen handelt es sich um einen pathologischen Zahnhartsubstanzverlust, der durch den chemischen Einfluss von Säuren oder KalziumChelatoren ohne die Beteiligung von Mikroorganismen entsteht. Die Säuren können intrinsischer oder extrinsischer Herkunft sein (ten Cate und Imfeld 1996). Je nach Ätiologie, die zumeist multifaktoriell bedingt ist, können Erosionen unterschiedlich lokalisiert und individuell schwach bis stark ausgeprägt sein (Eccles und Jenkins 1974).

Der erosive Prozess beginnt auf der Schmelzoberfläche des Zahnes und schreitet flächenhaft in die Tiefe voran. Dabei ist der schichtweise Zahnhartsubstanzverlust für den Betroffenen zunächst schmerzlos und nicht sichtbar, jedoch irreversibel. Bei einem fortschreitenden Verlauf ist zumeist auch das Dentin betroffen, was sich durch Hypersensibilitäten, Verfärbungen und deutlich sichtbare Defekte zeigt (Hotz 1987). Die beginnende Erosion zeichnet sich durch den Verlust der Perikymatien aus, woraus eine glatte und seidenmatte Oberfläche des Zahnes resultiert. Im weiteren Verlauf des erosiven Prozesses zeigen sich an vestibulären und oralen Glattflächen flache und matte Konkavitäten, die breiter als tief und deren Ränder abgerundet sind. Besonders im inzisalen, aber auch approximalen Zahnschmelz von erodierten Frontzähnen beobachtet man eine gesteigerte Transluzenz (Eccles und Jenkins 1974). Am marginalen Rand der Gingiva persistiert typischerweise eine intakte Schmelzleiste, die vermutlich dadurch zustande kommt, dass die Sulkusflüssigkeit ( $\mathrm{pH} 7,5$ bis 8,0) die Säure unmittelbar neutralisiert (Stephen et al. 1980). Alternativ wird diskutiert, dass Plaque-Reste den zervikalen Schmelz vor Erosionen schützen könnten (Lussi et al. 2006). Bei einem progredienten Verlauf der Erosion resultieren Veränderungen der Zahnform, die sich als muldenförmige Einziehungen auf der Vestibularfläche zeigen (Zipkin und McClure 1949). Das Erscheinungsbild der okklusalen Erosion imponiert anfänglich mit schüsselförmig ausgewaschenen Höckerspitzen. Wenn Füllungen in den betroffenen Zähnen vorhanden sind, stehen die Füllungsränder über das erodierte Oberflächenniveau des jeweiligen Zahnes. Das fortgeschrittene Stadium kann zum vollständigen Verlust der okklusalen Morphologie und der Anatomie des Zahnes führen, woraus eine Re- 
duktion der vertikalen Dimension resultiert (Ganss und Lussi 2014, Lussi et al. 2011).

Die Erosion zählt zur Gruppe der nichtkariösen Zahnhartsubstanzdefekte, zu der auch Abrasion, Attrition, Demastikation, Abfraktion und Resorption gehören (Imfeld 1996). Diese Defekte treten sowohl isoliert als auch kombiniert auf. Das kann dazu führen, dass die individuellen klinischen Erscheinungsbilder variieren und Diagnose und ätiologische Zuordnung erschwert sind (Putz und Attin 2002).

\subsection{Pathogenese}

Der Speichel ist bei neutralem pH-Wert eine an Hydroxylapatit übersättigte Lösung (Thylstrup und Fejerskov 1986). Der Hydroxylapatit des Zahnschmelzes steht mit den Kalzium- und Phosphationen des Speichels in einem chemischen Gleichgewicht. Sinkt der pH-Wert durch Zufuhr von Säure unter den für Zahnschmelz kritischen Wert von 5,3 bis 5,5 (Rytömaa et al. 1988, Thylstrup und Fejerskov 1996), ist der Speichel an Hydroxidionen untersättigt und Kalzium- und Phosphationen des Zahnschmelzes gehen entlang des Diffusionsgradienten in Lösung (Graf 1953). Komplexbildende Chelatoren (z. B. Zitronensäure), die eine Bindung mit Kalziumionen eingehen, fördern diesen Prozess und verstärken die Demineralisation (Brosowsky 1966). Innerhalb der Hydroxylapatitkristalle des Zahnschmelzes werden zuerst die Prismenscheiden, dann der Prismenkern und zuletzt die interprismatische Substanz aufgelöst (Meurman und Frank 1991). Erreicht die Erosion das Dentin, wird zuerst das peri-, dann das intertubuläre Dentin zerstört. So gehen oberflächlich die anorganischen Bestandteile des Dentins verloren, während die organische Matrix zunächst erhalten bleibt und freiliegt (Breschi et al. 2002, Ganss et al. 2010). In vitro zeigt sich eine gewisse Resistenz dieser freiliegenden und schützenden Kollagenschicht gegenüber abrasiven Vorgängen und fortschreitender Erosion (Ganss et al. 2009). Bei mechanischer Belastung resultiert in Abhängigkeit vom einwirkenden Druck aber eine Kompression der freiliegenden Kollagenschicht. Ferner degradieren Kollagenasen und andere proteolytisch wirksame Enzyme der Mundhöhle das demineralisierte Kollagen (Schlueter et al. 2010). Insgesamt handelt es sich bei dentalen Erosionen um 
eine irreversible und schichtweise Abtragung der Zahnhartsubstanzen (Meurman und ten Cate 1996).

\section{3 Ätiologie und Lokalisation}

Die Entstehung von dentalen Erosionen kann als multifaktorielles Geschehen betrachtet werden, wobei man drei Gruppen von Faktoren klassifiziert. Man unterscheidet extrinsische, intrinsische und biologische Einflüsse (Lussi et al. 2007), die im Folgenden genauer beschrieben werden sollen.

\subsubsection{Extrinsische Faktoren}

Saure Nahrungsmittel und Getränke, Medikamente, berufsbedingte Säureexposition und umweltbedingte Faktoren zählen zu den extrinsischen Faktoren, die eine Erosion auslösen können (Zero 1996). Erosionen, die durch extrinsische Faktoren hervorgerufen werden, sind zumeist fazial und okklusal lokalisiert (Eccles und Jenkins 1974).

$\mathrm{Zu}$ den erosiven Getränken und Nahrungsmitteln gehören Softdrinks, Limonaden, saure Fruchtsäfte und Früchte, Smoothies, isotonische Sportgetränke, Wein, saure Süßigkeiten, Salatdressings und Essigprodukte (Järvinen et al. 1991, Nahás Pires Corrêa et al. 2011, Blacker und Chadwick 2013). In der Literatur werden verschiedene Schwellenwerte für den pH-Wert angegeben, wonach Substanzen mit darunterliegenden $\mathrm{pH}-$ Werten als potentiell erosiv gelten (Stephan 1966: 4,0; Larsen und Nyvad 1999: 5,5). Die Erosivität einer sauren Substanz ist zudem abhängig von ihrem pKs-Wert. Dieser bemisst die Protonenabgabe während einer chemischen Reaktion (Rytömaa et al. 1988). Deshalb können Nahrungsmittel und Getränke mit ähnlichen $\mathrm{pH}-$ Werten unterschiedlich erosiv sein. Die Konzentration von Kalzium und Phosphat in einem Nahrungsmittel oder Getränk beeinflusst ebenfalls die Erosivität. Trotz eines niedrigen pH-Wertes von ca. 3,8 verursacht Joghurt aufgrund seines hohen Anteils an Kalzium und Phosphat keine Erosionen (Larsen und Nyvad 1999).

Ein erhöhtes Risiko für Erosionen besteht bei vegetarischer, veganer und rohköstlicher Ernährung. Diese Formen der Ernährung zeichnen sich durch einen erhöhten Konsum von Gemüse und Früchten aus und gelten gemeinhin als gesunder Lebensstil. Linkosalo und Markkanen (1985) zeigten, dass Vegetarier 
eine bedeutend höhere Prävalenz von Erosionen haben. Ganss et al. (1999) fanden heraus, dass Rohköstler häufiger und schwerer von Erosionen betroffen sind.

Ein Lebensstil, der sich durch ein hohes Maß an Gesundheitsbewusstsein auszeichnet, korreliert häufig auch mit einer überdurchschnittlich guten Mundhygiene (Graehn 1991). Mundhygieneprodukte, die Ethylendiamintetraessigsäure (EDTA) beinhalten oder einen niedrigen $\mathrm{pH}-$ Wert aufweisen, wie Listerine ${ }^{\circledR}$ und Hexetidin-Mundspüllösungen, können Erosionen begünstigen (Rytömaa et al. 1989, Pontefract et al. 2001). Der niedrige pH-Wert von fluoridhaltigen Mundhygieneprodukten stellt hier eine Ausnahme dar. Die kariespräventive Eigenschaft von Fluoriden basiert auf der Bildung einer an Kalziumfluorid reichen Deckschicht. Die Bildung dieser Kalziumfluoride hängt stark von der Verfügbarkeit von Kalzium ab. Je saurer ein Mundhygieneprodukt, desto mehr Kalzium kann aus dem Zahn oder aus dem Speichel gelöst und zur Bildung von Kalziumfluorid zur Verfügung gestellt werden. Dieses Kalziumfluorid-Präzipitat schützt die Zahnoberfläche vor Säureattacken (Attin et al. 1999).

Erosionen können auch mit der Einnahme verschiedener Medikamente assoziiert sein. Das Kauen von Acetylsalicylsäure und Vitamin-C-Präparaten kann zu Erosionen auf den Okklusalflächen führen (Sullivan und Kramer 1983). Der potentielle Zusammenhang von Asthma bronchiale und Erosionen wird in einigen Studien untersucht, da der saure $\mathrm{pH}$-Wert bestimmter Asthmasprays als erosionsfördernd diskutiert wird. Während Al-Dlaigan et al (2002) und McDerra et al. (1998) einen signifikanten Zusammenhang beobachteten, konnten Dugmore und Rock (2003b) dies nicht bestätigen. Medikamente, die die Speichelfließrate herabsetzen und deshalb Erosionen begünstigen, sind: Antihistaminika, Retinoide, Diuretika, Tranquilizer, Anti-Parkinson-Medikamente, Anti-HIV-Medikamente, Anti-Migräne-Medikamente, Sympathomimetika, Antiemetika, Antidepresssiva, Bronchodilatatoren, Muskelrelaxantien, Zytostatika und Antihypertonika (Wynn und Meiller 2001, Scully 2003).

$\mathrm{Zu}$ den Berufsgruppen mit einem erhöhten Risiko für Erosionen zählen Personen, die in der Batterie-Industrie und Galvanotechnik beschäftigt und dabei regelmäßig Schwefel- oder Salzsäuredämpfen ausgesetzt sind. Kim und Douglass (2003) stellten ein hohes Risiko für Erosionen durch die Exposition 
gegenüber säurehaltigen Dämpfen fest. Das Tragen von Gesichtsmasken mindert dieses Risiko. Erosionen, die durch säurehaltige Dämpfe entstehen, sind zumeist an den Labialflächen der mittleren oberen Frontzähne lokalisiert (Amin et al. 2001). Studien, die chloriertes Wasser als Risikofaktor untersuchen, zeigen bei Profischwimmern eine höhere Prävalenz für Erosionen verglichen mit den Kontrollgruppen (Centerwall et al. 1986, Buczkowska-Radlińska et al. 2013).

\subsubsection{Intrinsische Faktoren}

$\mathrm{Zu}$ den intrinsischen Faktoren zählen Magensäure und saurer Mageninhalt (Järvinen et al. 1988). Erkrankungen, die dazu führen, dass Magensaft wiederholt in die Mundhöhle gelangt, sind gastroösophagealer Reflux (gastroesophageal reflux desease, GERD), Essstörungen (Bulimia nervosa und Rumination) und chronisches Erbrechen als Folge anderer Erkrankungen oder Nebenwirkung einer Medikation (Scheutzel 1996). Ein weiterer Grund für häufiges Erbrechen ist eine Schwangerschaft. Erosionen, die durch intrinsische Faktoren hervorgerufen werden, sind vor allem auf den palatinalen und lingualen Flächen der Zähne lokalisiert (Bartlett et al. 1996, Shaw und O'Sullivan 2000).

Der pH-Wert von Magensäure liegt mit etwa 1,0 bis 1,5 weit unterhalb des kritischen Wertes für Zahnschmelz (Jones et al. 2002). Verglichen mit Erfrischungsgetränken hat Magensäure ein höheres erosives Potential und löst in vitro bei identischer Einwirkzeit mehr Kalzium aus den Zahnhartgeweben (Bartlett und Coward 2001).

Einige Studien untersuchen den Zusammenhang von gastroösophagealem Reflux und Erosionen (Benages et al. 2006, Di Fede et al. 2008, Correa et al. 2012). Benages et al. (2006) beobachteten, dass Reflux mit häufigeren und schwereren Erosionen einhergeht. Ein Symptom der Reflux-Erkrankung ist die Regurgitation (Janssens et al. 1986). Durch sie gelangt Mageninhalt aufgrund einer Ringmuskel-Insuffizienz zurück in den Ösophagus oder die Mundhöhle. Di Fede et al. (2008) stellten hingegen keinen Zusammenhang zwischen Reflux und Erosionen, jedoch zwischen Reflux und einer verminderten Speichelfließrate (Xerostomie) fest. Die Bulimie ist eine weitere Erkrankung, die im Zusammenhang mit dem Vorliegen von Erosionen diskutiert wird. Ein erhöhtes Risiko 
für Erosionen bei Bulimikern stellt in erster Linie das häufige Erbrechen dar (Bartlett und Coward 2001). In klinischen Studien zeigt sich, dass Bulimia nervosa auch mit einer verminderten Speichelfließrate assoziiert ist (Rytömaa et al. 1998, Ohrn und Angmar-Mansson 2000). Ebenfalls besteht eine Tendenz zu Alkohol-Abusus (Lilenfeld et al. 2000). Es wird ein Zusammenhang von Alkoholismus und Erosionen diskutiert. Alkoholiker konsumieren vermehrt saure Getränke und erbrechen häufiger (Robb und Smith 1990, Dukic et al. 2010).

Während einer Schwangerschaft sind Frauen einem erhöhten Risiko ausgesetzt, dentale Erosionen zu entwickeln. Bei einer Mehrzahl der Schwangeren tritt besonders in den ersten drei Monaten der Schwangerschaft eine chronische Übelkeit auf, die in einigen Fällen zu häufigem Erbrechen führt (Lacasse et al. 2008). Auch gastroösophagealer Reflux kann während einer Schwangerschaft gehäuft auftreten (Naumann et al. 2012). Patienten, bei denen eine Chemotherapie Anwendung findet, leiden häufig unter Übelkeit und chronischem Erbrechen (Jordan et al. 2015). Zusätzlich tritt eine verminderte Speichelfließrate als Nebenwirkung der Chemotherapie auf (Scully 2003). Antiemeti$\mathrm{ka}$, die zur Behandlung von CINV (chemotherapy-induced nausea and vomiting) eingesetzt werden, setzen ebenfalls die Speichelfließrate herab.

\subsubsection{Biologische Faktoren}

Zu den biologischen Faktoren zählen der Speichel, die Anatomie der Zähne, die Anatomie und Zusammensetzung der Zahnhartsubstanzen, die Okklusion und die Anatomie der oralen Weichgewebe (Zero 1996). Die Interaktion der biologischen Faktoren beeinflusst gemeinsam mit erosiven Substanzen und erosionsfördernden Verhaltensweisen im Zeitverlauf die Entwicklung erosiver Defekte (Zero und Lussi 2000).

Der Speichel gilt als wichtigster biologischer Faktor bei der Entstehung von Erosionen. Er verdünnt, neutralisiert und puffert Säuren. Üblicherweise wird die Speichelfließrate durch gustatorische Reize verstärkt (Engelen et al. 2003). Ein reduzierter Speichelfluss wird z. B. bei Personen, die mit Bestrahlung im Kopfund Halsbereich therapiert werden, beobachtet (Dreizen et al. 1977). Klinisch zeigt sich ein Zusammenhang zwischen geringer Speichelfließrate, verminderter Pufferkapazität und der Entstehung von dentalen Erosionen (Järvinen et al. 1991, Bartlett et al. 1998, González-Aragón et al. 2016). 
In der Literatur wird das Potential des Speichels zur Remineralisation von dentalen Erosionen diskutiert (Larsen und Fejerskov 1989, Attin et al. 2000, Hara et al. 2008). Erosionen sind durch den Speichel kaum remineralisierbar, da nach vollständiger Auflösung der Zahnoberfläche durch Säuren nur eine dünne demineralisierte Schicht zurückbleibt, die remineralisierbar wäre (Lussi et al. 2011).

Zudem variieren die Pufferkapazität und das Remineralisationspotential des Speichels lokal in der Mundhöhle. Der seröse Speichel der Glandula parotidea weist eine größere Pufferkapazität und ein höheres Remineralisationspotential auf, als der sero-muköse Speichel der Glandula submandibularis und der mukoseröse Speichel der Glandula sublingualis (Millward et al. 1997, Young und Khan 2002, Lussi et al. 2012).

Weiterhin ist der Speichel an der Bildung der Pellikel (aquired salivary pellicle) beteiligt, die sich größtenteils aus Speichelproteinen formiert und sich unmittelbar nach Zahnreinigung auf den Zahnoberflächen bildet. Neben Speichelproteinen finden sich in dieser semipermeablen Membran Proteine der gingivalen Sulkusflüssigkeit, des Blutes, der Wangenschleimhaut, der Nahrung und bakteriellen Ursprungs (Hannig und Joiner 2006). Studien zeigen, dass die Pellikel in Abhängigkeit von ihrer Dicke und Reifungszeit die Zahnhartsubstanzen bis zu einem gewissen Grad vor erosiven Säureangriffen schützen kann (Amaechi et al. 1999b, Hannig et al. 2003, Wiegand et al. 2008).

Eine weitere physiologische Einflussgröße für die Entstehung von dentalen Erosionen sind die Zusammensetzung und die Anatomie der Zahnhartsubstanzen. Der Zahnschmelz besteht zu 2 Gew.-\% aus organischer Substanz und zu 96 Gew.-\% aus anorganischen Mineralien, die in einem Gerüst aus hexagonalen Hydroxylapatit-Prismen verdichtet sind. Dentin setzt sich hingegen zu 20 Gew.-\% aus organischen und $70 \mathrm{Gew} .-\%$ aus anorganischen Bestandteilen zusammen (Schroeder 2000). Die Kristalle der anorganischen Matrix sind im Dentin lockerer angeordnet und kleiner als im Zahnschmelz. Zudem weist die anorganische Matrix des Dentins mehr Verunreinigungen in Form von Carbonat-, Natrium- und Magnesium-lonen als die anorganische Matrix des Schmelzes auf. Daraus resultiert, dass Dentin eine höhere Säurelöslichkeit zeigt als Schmelz (Shellis et al. 2014). Andererseits enthält das Dentin Kollagenfasern, 
die nach Demineralisation durch Säuren zunächst erhalten bleiben und für eine gewisse Zeit vor voranschreitender Erosion schützen. Solange die freiliegenden Kollagenfasern hydriert und vital sind, stellen sie eine Diffusionsbarriere für Säuren dar. Sie verhindern, dass weitere Mineralien aus dem darunterliegenden und noch intakten Dentin herausgelöst werden (Ganss et al. 2004, Hara et al. 2005).

\subsubsection{Die Interaktion von Erosion, Attrition und Abrasion}

Attrition und Abrasion der Zahnhartsubstanzen sind bis zu einem gewissen Grad als physiologische Zahnabnutzung zu verstehen, können aber pathologische Formen annehmen. Attrition wird als Zwei-Körper-Abrieb durch Zahn-ZuZahn-Kontakt ohne Beteiligung von Fremdkörpern, Abrasion als Drei-KörperAbrieb durch Zahn-zu-Fremdkörper-zu-Zahn-Kontakt definiert. Abrasionen, die durch Nahrungsmittel entstehen, werden Demastikationen genannt (Imfeld 1996).

Die Attrition beeinflusst als Zahn-Zu-Zahn-Kontakt die Progredienz der Erosion. In vitro zeigt sich, dass die mechanische Belastung erodierter Zahnoberflächen den Substanzverlust gegenüber der alleinigen Erosion verstärkt (Vieira et al. 2006). So können das Kauen, der Kontakt zu oralen Weichgeweben (z. B. Zunge) oder das Zähneputzen erodierter Zähne zu weiterem Zahnhartsubstanzverlust führen (Lewis und Smith 1973, Gregg et al. 2004, Wiegand et al. 2007).

\subsubsection{Besonderheiten im Milchgebiss}

Die Milchzähne unterscheiden sich im Hinblick auf ihre Größe und ihrer Anatomie des Zahnschmelzes von der bleibenden Dentition. Sie sind insgesamt kleiner als ihre jeweiligen permanenten Nachfolger und haben eine deutlich dünnere Schmelz-Schicht als die bleibenden Zähne (Grine 2005, Mahoney 2013). Histologisch finden sich im Zahnschmelz weitere Unterschiede zwischen der ersten und zweiten Dentition. Die Prismen im Milchzahn-Schmelz sind kleiner und weniger miteinander verbunden. Das lässt inn poröser erscheinen als den Schmelz der permanenten Zähne (Shellis 1984). Der organische Anteil im Schmelz der ersten Dentition variiert zwischen 0,7\% und 12\%, und zeigt damit eine größere Bandbreite als der organische Anteil des permanenten Schmelzes mit $0,4 \%$ bis $0,8 \%$ (Stack 1953). Insgesamt betrachtet ist der 
Zahnschmelz im Milchgebiss deutlich geringer mineralisiert und signifikant weicher als in der bleibenden Dentition (Wilson und Beynon 1989, Lussi et al. 2000, Johansson et al. 2001, Magalhães et al. 2009). Hunter et al. (2000) beobachteten in vitro, dass der erosive Prozess bei Milchzähnen schneller in das Dentin fortschreitet als bei bleibenden Zähnen.

Untersuchungen der Hypothese, dass Milchzähne anfälliger für Erosionen und Erosio-Abrasionen sind, zeigen unterschiedliche Ergebnisse. Amaechi et al. (1999a) bestätigten in vitro eine 1,5 Mal höhere Anfälligkeit für Erosionen im Milchgebiss, während Lippert et al. (2004) keinen Unterschied feststellten. Während Correr et al. (2007) durch einen Versuchsaufbau in vitro vergleichsweise stärkere Erosio-Abrasionen an Milchzähnen als an bleibenden Zähnen hervorriefen, fanden Attin et al. (2007) nur geringfügige Unterschiede.

\subsection{Epidemiologie}

\subsubsection{Erosionsindizes für epidemiologische Studien}

Zur Anwendung in epidemiologischen Untersuchungen werden seit den 1970er Jahren verschiedene Indizes für die Klassifikation von Erosionen vorgeschlagen. Laut Bardsley (2008) gelten die Indizes nach Eccles (1979, Tabelle 2.1) und Smith und Knight (1984, Tabelle 2.2) als Grundlage zur Klassifikation von nichtkariösen Zahnhartsubstanzdefekten, auf der viele weitere Indizes entwickelt wurden. 


\begin{tabular}{|c|c|c|}
\hline Grad & Zahnfläche & Kriterium \\
\hline $\mathbf{I}$ & & $\begin{array}{l}\text { Frühe Anzeichen von Erosion; Verlust von Perikymatien, glatte } \\
\text { Oberfläche, hauptsächlich auf den labialen Zahnflächen der } \\
\text { Schneide- und Eckzähne im Oberkiefer }\end{array}$ \\
\hline \multirow[t]{2}{*}{ II } & Fazial & Dentinbeteiligung von weniger als $1 / 3$ der Zahnfläche \\
\hline & & $\begin{array}{l}\text { Typ 1: ovale oder halbmondförmige konkave Läsion im zervi- } \\
\text { kalen Bereich, die sich deutlich von einem keilförmigen Defekt } \\
\text { unterscheidet } \\
\text { Typ 2: Unregelmäßige Läsion auf der gesamten Zahnfläche, } \\
\text { die durch den Verlust von Schmelz wie ausgestanzt erscheint }\end{array}$ \\
\hline IIla & Fazial & $\begin{array}{l}\text { Noch ausgedehntere Dentinbeteiligung, besonders an den } \\
\text { Frontzähnen; häufiger ist ein Großteil der Zahnfläche betroffen, } \\
\text { aber auch enger begrenzte Läsionen, die wie ausgewaschen } \\
\text { erscheinen, treten auf }\end{array}$ \\
\hline IIIb & $\begin{array}{l}\text { Lingual/ } \\
\text { Palatinal }\end{array}$ & $\begin{array}{l}\text { Mehr als 1/3 der Zahnfläche ist betroffen; Inzisalkanten werden } \\
\text { aufgrund von Dentinverlust transluzent; das Dentin wirkt glatt } \\
\text { und manchmal abgeflacht oder ausgewaschen; gingivale und } \\
\text { approximale Ränder erscheinen weiß und angeätzt }\end{array}$ \\
\hline IIIc & $\begin{array}{l}\text { Inzisal/ } \\
\text { Okklusal }\end{array}$ & $\begin{array}{l}\text { Inzisalkanten oder okklusale Höcker sind bis in das Dentin } \\
\text { flach oder schüsselförmig ausgewaschen, Füllungsränder er- } \\
\text { scheinen höher als die sie umgebende Zahnoberfläche, Inzi- } \\
\text { salkanten sind aufgrund des unterminierten Schmelzes } \\
\text { transluzent }\end{array}$ \\
\hline IIId & $\begin{array}{l}\text { Alle } \\
\text { Flächen }\end{array}$ & $\begin{array}{l}\text { Stark betroffene Zähne, wobei sowohl labiale als auch linguale } \\
\text { bzw. palatinale Flächen ausgedehnt beteiligt sind }\end{array}$ \\
\hline
\end{tabular}

Tabelle 2.1: Erosions-Index nach Eccles 1979. 


\begin{tabular}{|c|c|c|}
\hline Grad & Zahnfläche & Kriterium \\
\hline \multirow[t]{2}{*}{0} & $\begin{array}{l}\text { Bukkal, Oral, } \\
\text { Okklusal, Inzisal }\end{array}$ & Kein Verlust der Oberflächenstruktur im Schmelz \\
\hline & Zervikal & Kein Verlust der Kontur \\
\hline \multirow[t]{2}{*}{1} & $\begin{array}{l}\text { Bukkal, Oral, } \\
\text { Okklusal, Inzisal }\end{array}$ & Verlust der Oberflächenstruktur im Schmelz \\
\hline & Zervikal & Minimaler Verlust der Kontur \\
\hline \multirow{3}{*}{2} & $\begin{array}{l}\text { Bukkal, Oral, } \\
\text { Okklusal }\end{array}$ & $\begin{array}{l}\text { Schmelzverlust mit freiliegendem Dentin auf weniger als } \\
1 / 3 \text { der Oberfläche }\end{array}$ \\
\hline & Inzisal & Schmelzverlust mit freiliegendem Dentin \\
\hline & Zervikal & Defekt von weniger als $1 \mathrm{~mm}$ Tiefe \\
\hline \multirow{3}{*}{3} & $\begin{array}{l}\text { Bukkal, Oral, } \\
\text { Okklusal }\end{array}$ & $\begin{array}{l}\text { Schmelzverlust mit freiliegendem Dentin auf mehr als } 1 / 3 \\
\text { der Oberfläche }\end{array}$ \\
\hline & Inzisal & Schmelzverlust mit tief freiliegendem Dentin \\
\hline & Zervikal & Defekt von 1 bis $2 \mathrm{~mm}$ Tiefe \\
\hline \multirow{3}{*}{4} & $\begin{array}{l}\text { Bukkal, Oral, } \\
\text { Okklusal }\end{array}$ & $\begin{array}{l}\text { Kompletter Schmelzverlust oder Pulpafreilegung oder } \\
\text { Exposition von Sekundärdentin }\end{array}$ \\
\hline & Inzisal & Pulpa- oder Sekundärdentin-Freilegung \\
\hline & Zervikal & $\begin{array}{l}\text { Defekt ab } 2 \text { mm Tiefe mit Pulpa- oder Sekundärdentin- } \\
\text { Freilegung }\end{array}$ \\
\hline
\end{tabular}

Tabelle 2.2: „Tooth Wear Index“ nach Smith und Knight 1984.

Der Index nach O'Sullivan (2000) wurde speziell für die Anwendung im Milchgebiss und der frühen 2. Dentition entwickelt. Er zeichnet sich durch eine große Genauigkeit in der Bewertung von dentalen Erosionen aus (Tabelle 3.1, Kapitel 3). Ein relativ neuer Index zur Klassifikation von dentalen Erosionen bei Kindern und Erwachsenen wird von Bartlett et al. (2008) in der Literatur vorgestellt (Tabelle 3.2, Kapitel 3). Die Autoren schlagen den BEWE-Index (Basic Erosive Wear Examination) als Grundlage zur Entwicklung eines international standardisierten Index vor, der die Vergleichbarkeit von Studien untereinander verbessern soll. Aufgrund seiner Einfachheit soll auch ein Vergleich mit Studien mög- 
lich sein, innerhalb derer zur Klassifikation von Erosionen andere Indizes verwendet wurden.

\subsubsection{Prävalenz bei Erwachsenen, Jugendlichen und Kindern}

Die Prävalenz von dentalen Erosionen bei Erwachsenen wird seit den 1970er Jahren, die Prävalenz von Kindern und Jugendlichen seit den 1990er Jahren vielfach untersucht. Aufgrund unterschiedlicher Indizes und Untersuchungsmethoden lassen sich die heterogenen Ergebnisse nur schwer miteinander vergleichen. Ein Großteil der Daten basiert auf klinischen Untersuchungen und wurde anhand der Beurteilung der gesamten Dentition oder einzelner Markerzähne gewonnen. Es wurden aber auch epidemiologische Studien auf der Grundlage von extrahierten Zähnen (Sognnaes et al. 1972), Modellen (Ganss et al. 2001) oder Fotos von Modellen (Khan et al. 2001) durchgeführt.

Im Folgenden soll der Fokus auf den Studien liegen, die zur Ermittlung der Prävalenz im Rahmen einer klinischen Untersuchung die gesamte Dentition und alle Zahnflächen bewerteten (Tabellen 2.3 bis 2.6).

Für Erwachsene wird eine Prävalenz von 5\% (Järvinen et al. 1991) bis 100\% (Donachie und Walls 1995, Bartlett et al. 2011) angegeben (Tabelle 2.3). 


\begin{tabular}{|c|c|c|c|c|}
\hline Autoren und Jahr & $\mathbf{N}$ & Alter & Index & Prävalenz \\
\hline $\begin{array}{l}\text { Xhonga und Valdmanis } \\
1983\end{array}$ & 527 & $14-88$ & Eigene Kriterien & $25 \%$ \\
\hline Järvinen et al. 1991 & 100 & $17-83$ & Eccles und Jenkins & $5 \%$ \\
\hline Lussi et al. 1991 & 391 & $\begin{array}{l}26-30 \\
46-50\end{array}$ & Lussi & $\begin{array}{l}35,6 \% \\
42,6 \%\end{array}$ \\
\hline Donachie und Walls 1995 & 586 & $45-$ & TWI (Tooth Wear Index) & $100 \%$ \\
\hline Smith und Robb 1996 & $\begin{array}{l}210 \\
195 \\
234 \\
154 \\
126 \\
88\end{array}$ & $\begin{array}{c}15-26 \\
26-35 \\
36-45 \\
46-55 \\
56-65 \\
65-\end{array}$ & TWI & $\begin{array}{l}5,7 \% * \\
3,4 \% * \\
3,8 \% \text { * } \\
4,6 \% * \\
8,2 \% * \\
8,8 \% \text { * }\end{array}$ \\
\hline Jaeggi et al. 1999 & 417 & $19-25$ & Lussi & $82 \%$ \\
\hline Schiffner et al. 2002 & $\begin{array}{c}655 \\
1027\end{array}$ & $\begin{array}{l}35-44 \\
65-74\end{array}$ & Lussi & $\begin{array}{c}10,7 \% \\
7,9 \%\end{array}$ \\
\hline Bartlett et al. 2011 & 1010 & $18-30$ & TWI & $100 \%$ \\
\hline Mulic et al. 2012 & 1456 & 18 & $\begin{array}{l}\text { VEDE (Visual Erosion } \\
\text { Dental Examination) }\end{array}$ & $38 \%$ \\
\hline Manaf et al. 2012 & 150 & $19-24$ & $\begin{array}{c}\text { BEWE (Basic Erosive } \\
\text { Wear Examination) }\end{array}$ & $68 \%$ \\
\hline Vered et al. 2014 & 500 & $\begin{array}{l}15-18 \\
25-28 \\
35-38 \\
45-48 \\
55-60\end{array}$ & BEWE & $\begin{array}{l}36,6 \% \\
42,0 \% \\
55,8 \% \\
53,1 \% \\
61,9 \%\end{array}$ \\
\hline Zebrauskas et al.2014 & 56 & $18-25$ & Lussi & $50 \%$ \\
\hline Chu et al. 2015 & 600 & $18-21$ & BEWE & $44 \%$ \\
\hline Kitasako et al. 2015 & 1108 & $15-89$ & Modifizierter TWI & $26,1 \%$ \\
\hline
\end{tabular}

Tabelle 2.3: Prävalenz dentaler Erosionen bei Erwachsenen in chronologischer Reihenfolge. $\mathbf{N}=$ Anzahl der Probanden, Alter in Jahren, \% - Prozent der Studienteilnehmer, \%* Prozent der Zahnflächen. 
Für Kinder und Jugendliche zwischen 10 und 17 Jahren finden sich Angaben von 1,4\% (Kirthiga et al. 2015) bis 100\% (Al-Dlaigan et al. 2001).

\begin{tabular}{|c|c|c|c|c|}
\hline Autoren und Jahr & $\mathbf{N}$ & Alter & Index & Prävalenz \\
\hline Al-Dlaigan et al. 2001 & 418 & 14 & Modifizierter TWI & $100 \%$ \\
\hline \multirow{2}{*}{ Van Rijkom et al. 2002} & 345 & $10-13$ & \multirow{2}{*}{ Modifizierter Lussi } & $3 \%$ \\
\hline & 400 & $15-16$ & & $30 \%$ \\
\hline Arnadottir et al. 2003 & 278 & 15 & Modifizierter Lussi & $21,6 \%$ \\
\hline Caglar et al. 2005 & 153 & 11 & O'Sullivan & $28 \%$ \\
\hline Larsen et al. 2005 & 558 & $15-17$ & Modifizierter Lussi & $14 \%$ ** \\
\hline Correr et al. 2009 & 389 & 12 & O'Sullivan & $26 \%$ \\
\hline \multirow{5}{*}{ El-Aidi et al. 2010} & \multirow{5}{*}{622} & 11 & \multirow{5}{*}{ Modifizierter Lussi } & $30,4 \%$ \\
\hline & & 12 & & $38,3 \%$ \\
\hline & & 13 & & $40,6 \%$ \\
\hline & & 14 & & $42,6 \%$ \\
\hline & & 15 & & $44,2 \%$ \\
\hline \multirow{2}{*}{ Caglar et al. 2011} & \multirow{2}{*}{83} & $7-11$ & \multirow{2}{*}{ Lussi } & $47,4 \%$ \\
\hline & & $12-14$ & & $52,6 \%$ \\
\hline $\begin{array}{l}\text { Nahás Pirres Côrrea et al. } \\
2011\end{array}$ & 232 & $2-20$ & $\begin{array}{c}\text { Modifizierter Index nach } \\
\text { O'Brien }\end{array}$ & $25,4 \%$ \\
\hline Kaczmarek et al. 2012 & 180 & 15 & BEWE & $36,1 \%$ \\
\hline Kumar et al. 2013 & 605 & $11-14$ & O‘Sullivan & $8,9 \%$ \\
\hline Zhang et al. 2014 & 600 & 12 & BEWE & $75 \%$ \\
\hline Zebrauskas et al.2014 & 76 & $12-17$ & Lussi & $25 \%$ \\
\hline Alves et al. 2015 & 1528 & 12 & BEWE & $15 \%$ \\
\hline Muller-Bolla et al. 2015 & 339 & 14 & BEWE & $39 \% * * *$ \\
\hline Kirthiga et al. 2015 & 2000 & $11-16$ & O'Sullivan & $1,4 \%$ \\
\hline
\end{tabular}




\begin{tabular}{|l|c|c|c|c|}
\hline Autoren und Jahr & N & Alter & Index & Prävalenz \\
\hline $\begin{array}{l}\text { Alvarez Loureiro et al. } \\
2015\end{array}$ & 1136 & 12 & BEWE & $52,9 \%$ \\
\hline $\begin{array}{l}\text { González-Aragón et al. } \\
2016\end{array}$ & 417 & $14-19$ & Lussi & $31,7 \%$ \\
\hline
\end{tabular}

Tabelle 2.4: Prävalenz dentaler Erosionen bei Kindern und Jugendlichen zwischen 10 und 17 Jahren in chronologischer Reihenfolge. $\mathbf{N}=$ Anzahl der Probanden, Alter in Jahren, \% - Prozent der Studienteilnehmer, \%* - Prozent der Erosionen mit Dentinbeteiligung, $\%^{* *}$ - Prozent der Studienteilnehmer mit mehr als 3 betroffenen Zahnflächen, $\%^{* * *}$ Prozent der Studienteilnehmer mit einem BEWE-Summenscore $\geq 3$. 
Die Prävalenz von Erosionen bei Kindern bis 9 Jahre variiert zwischen 0,6\% (Moimaz et al. 2013) und 100\% (Jaeggi und Lussi 2004). Studien zur Prävalenz in dieser Altersgruppe, deren Ergebnisse auf der Bewertung von Milchzähnen und zum Teil der frühen zweiten Dentition basieren, werden in Tabelle 2.5 vorgestellt.

\begin{tabular}{|c|c|c|c|c|c|}
\hline $\begin{array}{l}\text { Autoren und } \\
\text { Jahr }\end{array}$ & $\mathbf{N}$ & Alter & Index & Prävalenz & Bewertete Dentition \\
\hline $\begin{array}{l}\text { Jaeggi und } \\
\text { Lussi } 2004\end{array}$ & 42 & $5-9$ & Lussi & $\begin{array}{c}100 \% * \\
14,3 \% \text { ** }\end{array}$ & $\begin{array}{l}\text { Milchgebiss und } \\
\text { bleibende Zähne }\end{array}$ \\
\hline $\begin{array}{l}\text { Wiegand et al. } \\
2006\end{array}$ & 463 & $2-7$ & O‘Sullivan & $\begin{array}{l}32 \% * \\
0 \% \text { ** }\end{array}$ & $\begin{array}{l}\text { Milchgebiss und } \\
\text { bleibende Zähne }\end{array}$ \\
\hline Taji et al. 2010 & 128 & $2-4$ & $\begin{array}{l}\text { Modifizierter } \\
\text { Index nach } \\
\text { Aine }\end{array}$ & $75 \%$ & Milchgebiss \\
\hline $\begin{array}{l}\text { Gatou und } \\
\text { Mamai-Homata } \\
2012\end{array}$ & 243 & $5-7$ & TWI & $98,4 \%$ & Milchgebiss \\
\hline $\begin{array}{l}\text { Moimaz et al. } \\
2013\end{array}$ & 1993 & $4-6$ & TWI & $0,6 \%$ & Milchgebiss \\
\hline $\begin{array}{l}\text { Mantonanaki et } \\
\text { al. } 2013\end{array}$ & 605 & 5 & BEWE & $78,8 \%$ & Milchgebiss \\
\hline Tao et al. 2015 & 1837 & $3-6$ & O‘Sullivan & $15,1 \%$ & Milchgebiss \\
\hline $\begin{array}{l}\text { Huang et al. } \\
2015\end{array}$ & 154 & $\begin{array}{l}2 \\
3 \\
4\end{array}$ & $\begin{array}{c}\text { Modifizierter } \\
\text { TWI }\end{array}$ & $\begin{array}{l}0 \% \\
7 \% \\
33 \%\end{array}$ & Milchgebiss \\
\hline
\end{tabular}

Tabelle 2.5: Prävalenz dentaler Erosionen bei Kindern bis einschließlich 9 Jahre in chronologischer Reihenfolge. $\mathbf{N}=$ Anzahl der Probanden, Alter in Jahren, \% - Prozent der Studienteilnehmer, \%* Prävalenz im Milchgebiss, \%** Prävalenz im bleibenden Gebiss.

\subsubsection{Inzidenz und Progression}

Ganss et al. (2001) führten eine Studie zur Prävalenz und Inzidenz an Zähnen der bleibenden Dentition anhand von kieferorthopädischen Studienmodellen 
durch. Sie stellten eine Inzidenz von 18,7\% (Inzidenzrate: 4,1\%) innerhalb von fünf Jahren fest.

Dugmore und Rock (2003a) untersuchten die bleibenden Zähne von 1308 12jährigen Schülern. Sie ermittelten für einen Zeitraum von zwei Jahren eine Inzidenz von 28,1\% (Inzidenzrate: 16,5\%), und bei 26,8\% der Schüler eine Progredienz der dentalen Erosionen.

El-Aidi et al. (2010) führten eine Studie mit 622 teilnehmenden Jugendlichen durch, deren bleibende Zähne untersucht wurden. Die Inzidenz bei 11-jährigen Kindern lag bei 26,5\% (1,5 Jahre) bzw. 27,3\% (3 Jahre). Dies entspricht Inzidenzraten von 20,5\% bzw. 10,6\%. Die Progression lag bei 36,8\% (1,5 Jahre) und 56,3\% (3 Jahre). Die Inzidenz bei 12-jährigen Kindern lag bei 15,8\% (1,5 Jahre) und bei 20,2\% (3 Jahre). Dies entspricht Inzidenzraten von 11,5\% bzw. 7,5\%. Die Progression lag bei 29\% (1,5 Jahre) und 44,9\% (3 Jahre).

Huang et al. (2015) untersuchten die Milchzähne von 154 Kindern und stellten innerhalb von 2 Jahren eine Inzidenz von 33\% fest. Dies entspricht einer Inzidenzrate von $20,3 \%$.

Lussi et al. (1991) untersuchten die bleibenden Zähne von 55 Probanden. Sie stellten in der Altersgruppe der 26- bis 32-Jährigen eine Zunahme von 5\% und in der Altersgruppe der 46 - bis 50 -Jährigen von $18 \%$ für okklusale DentinErosionen nach sechs Jahren fest. Sie zeigten, dass sich die okklusalen DentinErosionen innerhalb von sechs Jahren bei den 46- bis 50-Jährigen verdreifacht hatten. Bei den 46- bis 50-Jährigen war die Progression deutlich ausgeprägter als bei den 26- bis 32-Jährigen.

\subsubsection{Risikofaktoren}

In den verschiedenen epidemiologischen Studien zur Prävalenz und Inzidenz bei Kindern, Jugendlichen und Erwachsenen finden sich zumeist auch Risikofaktoranalysen zur Klärung der Ätiologie von dentalen Erosionen.

Der Konsum von Softdrinks (Järvinen et al. 1991, Al-Majed et al. 2002, Harding et al. 2003, Murakami et al 2011, Mulic et al. 2012, Gatou und Mamai-Homata 2012) und sauren Fruchtsäften (Luo et al. 2005, Mulic et al. 2012, Zhang et al. 2014) wird oft als signifikanter Risikofaktor für Erosionen identifiziert. Der 
Konsum von sauren Getränken zur Schlafenszeit (Al-Majed et al. 2002) korreliert ebenso mit dem Auftreten von dentalen Erosionen wie das Hin- und Herschwenken von sauren Getränken im Mund vor dem Schlucken (Alvarez Loureiro et al. 2015). Je länger ein saures Getränk im Mund gehalten wird, desto größer ist das Risiko, dentale Erosionen zu entwickeln (Al-Majed et al. 2002). Neben sauren Getränken werden auch Obst und Früchte (Vered et al. 2014, Kitasako et al. 2015, Alves et al. 2015), Essig (Järvinen et al. 1991, Tao et al. 2015) sowie saure Süßigkeiten (Muller-Bolla et al. 2015) als Risikofaktoren für Erosionen identifiziert.

Das Mundhygieneverhalten hat ebenfalls einen Einfluss auf die Entstehung von Erosionen. Mulic et al. (2012) und Muller-Bolla et al. (2015) stellten einen positiven Zusammenhang zwischen schlechter Mundhygiene und dem Auftreten von Erosionen fest. Mantonanaki et al. (2013) kamen in ihrer Studie zu einem entgegengesetzten Ergebnis. Wiegand et al. (2006) zeigten, dass Kinder zwischen 2 und 7 Jahren, die ihre Zähne selbst putzen und nicht von den Eltern nachgeputzt bekommen, ein höheres Risiko haben, dentale Erosionen zu entwickeln.

Al-Malik et al. (2002) und Zhang et al. (2014) untersuchten die Korrelation von Karies und dentalen Erosionen. Beide Studien zeigen, dass Probanden mit Karieserfahrung deutlich mehr Erosionen aufweisen. Der sozioökonomische Status wird in vielen Studien als potentielle Einflussgröße für Erosionen untersucht. Die Ergebnisse sind teilweise widersprüchlich. Einige Studien zeigen, dass Probanden aus einem niedrigen sozioökonomischen Umfeld signifikant mehr Erosionen haben (Harding et al. 2003, Muller-Bolla et al. 2015). Im Gegensatz dazu stellen andere Studien fest, dass ein hoher sozioökonomischer Status mit signifikant mehr Erosionen einhergeht (Millward et al. 1994, Luo et al. 2005). Mantonanaki et al. (2013) identifizierten sowohl ein niedriges Bildungsniveau der Mutter als auch ein hohes Familieneinkommen als Risikofaktor für Erosionen bei 5-jährigen Kindern.

Zahlreiche Studien kommen zu dem Ergebnis, dass das Geschlecht der Studienteilnehmer mit der Häufigkeit und dem Schweregrad von Erosionen korreliert. Jungen und Männer sind signifikant häufiger von Erosionen betroffen als Mädchen und Frauen (Bere et al. 2008, Hasselkvist et al. 2010, El-Aidi et al. 2010, Gatou und Mamai-Homata 2012). Mulic et al. (2012), Gatou und Mamai- 
Homata (2012) und Alvarez Loureiro et al. (2015) zeigten außerdem, dass männliche Probanden schwerere Erosionen haben. Wenige Studien beobachten hingegen, dass das weibliche Geschlecht signifikant häufiger von Erosionen betroffen ist (Huew et al. 2012, Picos et al. 2013).

Einige Studien zeigen, dass das Risiko für Erosionen mit steigendem Alter zunimmt (Wiegand et al. 2006, Taji et al. 2010, Murakami et al 2011, Gatou und Mamai-Homata 2012). Ganss et al. (2001) stellten fest, dass Erosionen im Milchgebiss ein signifikantes Risiko für die Entwicklung von Erosionen im bleibenden Gebiss darstellen.

Bardsley et al. (2004) stellten Trinkwasser-Fluoridierung und den zweimaligen täglichen Gebrauch von fluoridierter Zahnpasta als signifikant protektive Einflussgröße bei der Entstehung von Erosionen fest. Die Frequenz des Milchkonsums ist ebenfalls mit einer geringeren Prävalenz für Erosionen oder weniger schweren Erosionen assoziiert (Nahás Pires Corrêa et al. 2011, Wiegand et al. 2006).

Tabelle 2.6 gibt abschließend eine Übersicht über in epidemiologischen Untersuchungen identifizierte Risikofaktoren bei Kindern zwischen 2 und 9 Jahren. 


\begin{tabular}{|c|c|c|c|c|}
\hline $\begin{array}{l}\text { Autoren und } \\
\text { Jahr }\end{array}$ & $\mathbf{N}$ & Land & Alter & $\begin{array}{l}\text { Identifizierte Risikofaktoren / signi- } \\
\text { fikante Ergebnisse }\end{array}$ \\
\hline $\begin{array}{l}\text { Millward et } \\
\text { al. } 1994\end{array}$ & 178 & $\begin{array}{l}\text { Groß- } \\
\text { Britannien }\end{array}$ & 4 & - Hoher sozioökonomischer Status \\
\hline $\begin{array}{l}\text { Al-Malik et al. } \\
\qquad 2002\end{array}$ & 987 & $\begin{array}{l}\text { Saudi- } \\
\text { Arabien }\end{array}$ & $2-5$ & - Karies \\
\hline $\begin{array}{l}\text { Al-Majed et } \\
\text { al. } 2002\end{array}$ & 354 & $\begin{array}{l}\text { Saudi- } \\
\text { Arabien }\end{array}$ & $5-6$ & $\begin{array}{l}\text { - Softdrinks zur Schlafenszeit } \\
\text { - Dauer, während der Getränke vor } \\
\text { dem Schlucken im Mund gehalten } \\
\text { werden }\end{array}$ \\
\hline $\begin{array}{l}\text { Harding et al. } \\
\qquad 2003\end{array}$ & 202 & Irland & 5 & $\begin{array}{l}\text { - Fruchtmus, Softdrinks } \geq 1 / \text { Tag } \\
\text { - Niedriges Familieneinkommen }\end{array}$ \\
\hline $\begin{array}{l}\text { Luo et al. } \\
\qquad 2005\end{array}$ & 1949 & China & $3-5$ & $\begin{array}{l}\text { - Hoher Bildungsgrad der Eltern } \\
\text { - Fruchtsaft via Fläschchen } \\
\text { - Fruchtsaft zur Schlafenszeit }\end{array}$ \\
\hline $\begin{array}{l}\text { Wiegand et } \\
\text { al. } 2006\end{array}$ & 463 & $\begin{array}{l}\text { Deutsch- } \\
\text { land }\end{array}$ & $2-7$ & $\begin{array}{l}\text { - Erosionen nehmen mit dem Alter zu } \\
\text { - Jungen > Mädchen } \\
\text { - Milch protektiv } \\
\text { - Kinder putzen selbst Zähne ohne } \\
\text { Nachputzen der Eltern: höheres Ri- } \\
\text { siko für Erosionen }\end{array}$ \\
\hline $\begin{array}{c}\text { Taji et al. } \\
2010\end{array}$ & 128 & Australien & $2-4$ & $\begin{array}{l}\text { - Schwere der Erosionen nimmt mit } \\
\text { zunehmendem Alter zu }\end{array}$ \\
\hline $\begin{array}{l}\text { Murakami et } \\
\text { al } 2011\end{array}$ & 967 & Brasilien & $3-4$ & $\begin{array}{l}\text { - Softdrinks } 2 / \text { Tag oder }>3 / \text { Tag } \\
\text { - Gastro-Ösophagealer Reflux } \\
\text { - Erosionen nehmen mit dem Alter zu }\end{array}$ \\
\hline $\begin{array}{c}\text { Gatou und } \\
\text { Mamai- } \\
\text { Homata } 2012\end{array}$ & 243 & $\begin{array}{l}\text { Griechen- } \\
\text { land }\end{array}$ & $5-7$ & $\begin{array}{l}\text { - Softdrinks: Prävalenz und Schwere } \\
\text { - Jungen > Mädchen } \\
\text { - Jungen: schwerere Erosionen } \\
\text { - Immigranten > Griechen } \\
\text { - Erosionen nehmen mit dem Alter zu }\end{array}$ \\
\hline
\end{tabular}




\begin{tabular}{|c|c|c|c|l|}
\hline $\begin{array}{c}\text { Autoren und } \\
\text { Jahr }\end{array}$ & N & Land & Alter & $\begin{array}{l}\text { Identifizierte Risikofaktoren / signi- } \\
\text { fikante Ergebnisse }\end{array}$ \\
\hline $\begin{array}{c}\text { Nayak et al. } \\
2012\end{array}$ & 1002 & Indien & 5 & $\begin{array}{l}\text { - Konsumhäufigkeit von Säften, roher } \\
\text { Mango, Tamarindenfrucht, Stachel- } \\
\text { beeren und Buttermilch } \\
\text { - Zähneputzen } \geq 2 / T a g\end{array}$ \\
\hline $\begin{array}{c}\text { Mantonanaki } \\
\text { et al. } 2013\end{array}$ & 605 & $\begin{array}{c}\text { Griechen- } \\
\text { land }\end{array}$ & 5 & $\begin{array}{l}\text { - Gute Mundhygiene } \\
\text { - Niedriger Bildungsgrad der Mutter } \\
\text { - Hohes Familieneinkommen }\end{array}$ \\
\hline $\begin{array}{c}\text { Tao et al. } \\
2015\end{array}$ & 1837 & China & $3-6$ & $\begin{array}{l}\text { - Essig-/Kaffee-/Tee-Konsum } \\
\text { - Hoher Bildungsgrad der Mutter } \\
\text { - Geburtsort } \\
\text { - Regurgitation }\end{array}$ \\
\hline $\begin{array}{c}\text { Huang et al. } \\
2015\end{array}$ & 154 & Australien & $2-4$ & $\begin{array}{l}\text { - Nahrungsaufnahme via Fläschchen } \\
\text { - Erosionen nehmen mit dem Alter zu }\end{array}$ \\
\hline
\end{tabular}

Tabelle 2.6: Epidemiologische Studien bei Kindern zwischen 2 und 9 Jahren, die eine Risikofaktoranalyse enthalten (in chronologischer Reihenfolge). $\mathbf{N}=$ Anzahl der Probanden, Alter in Jahren. 


\section{Probanden und Methoden}

\subsection{Kontaktaufnahme und Probandengut}

Vor der Durchführung der Untersuchung wurde die Studie von der Ethikkommission der Universität Göttingen begutachtet und unter der Nummer 8/11/14 genehmigt.

Im Rahmen der vorliegenden Studie wurden 62 Kindergärten mit insgesamt 2800 Kindergartenplätzen im Stadtgebiet von Göttingen eingeladen, an der Untersuchung teilzunehmen. Nach persönlicher Vorstellung der Untersucherin, Aufklärung über das Ziel der Studie und Information über den Ablauf der Untersuchung willigten 27 Einrichtungen ein, an der Studie teilzunehmen. Vor Beginn der klinischen Untersuchung wurden alle Eltern der 3- bis 7-jährigen Kinder über Ablauf und Ziel der Studie mündlich und schriftlich informiert; die Erziehungsberechtigten von 777 Kindern gaben $\mathrm{lhr}$ schriftliches Einverständnis zur Teilnahme an der Studie.

Die Eltern wurden gebeten, auf einem pseudonymisierten Anamnesebogen Allgemeinerkrankungen, Ernährungs- und Mundhygienegewohnheiten ihres Kindes anzugeben. Die Erzieherinnen und Erzieher in den Kindergärten wurden gebeten, einen Fragebogen bezüglich Ernährung und Mundhygiene in ihrer Einrichtung zu beantworten.

\subsection{Untersuchung}

An der klinischen Untersuchung nahmen 777 Kinder teil. Alle Kinder wurden unter standardisierten Bedingungen von einer durch die Leiterin der Studie intensiv kalibrierten Zahnärztin unter Zuhilfenahme einer transportablen Leuchte und Mundspiegel in den Kindergärten untersucht. Die Dokumentation erfolgte durch einen eingewiesenen Assistenten pseudonymisiert auf einem Auswertungsbogen.

\subsection{Erosions-Indizes}

Zur klinischen Untersuchung wurde der O'Sullivan-Index (O'Sullivan et al. 2000) herangezogen, der in seiner Anwendung einen Vergleich zu den in Göttingen erhobenen Daten von Wiegand et al. (2006) ermöglichte. Der O'Sullivan-Index (Tabelle 3.1) wurde speziell für die Anwendung im Milchgebiss und der frühen 
2. Dentition entwickelt und zeichnet sich durch eine große Genauigkeit in der Bewertung von dentalen Erosionen aus. Jedem Zahn wird bei der Bewertung ein dreiteiliger Code zugewiesen, bestehend aus Fläche, Schweregrad und Größe der betroffenen Fläche.

\begin{tabular}{|c|c|}
\hline Fläche & Kriterium \\
\hline Code A & Ausschließlich labial oder bukkal \\
\hline Code B & Ausschließlich lingual oder palatinal \\
\hline Code C & Ausschließlich okklusal oder inzisal \\
\hline Code D & Labial und inzisal/okklusal \\
\hline Code $\mathbf{E}$ & Lingual und inzisal/okklusal \\
\hline Code F & Multiple Flächen \\
\hline Schweregrad & Kriterium \\
\hline Code 0 & Normaler Schmelz \\
\hline Code 1 & $\begin{array}{l}\text { Seidenglänzende Schmelzoberfläche ohne Konti- } \\
\text { nuitätsverlust }\end{array}$ \\
\hline Code 2 & Kontinuitätsverlust der Schmelzoberfläche \\
\hline Code 3 & $\begin{array}{l}\text { Kontinuitätsverlust der Schmelz- und Dentinober- } \\
\text { fläche / Schmelz-Dentin-Grenze sichtbar }\end{array}$ \\
\hline Code 4 & $\begin{array}{l}\text { Schmelz- und Dentinverlust unterhalb der } \\
\text { Schmelz-Dentin-Grenze }\end{array}$ \\
\hline Code 5 & Schmelz und Dentinverlust mit Pulpafreilegung \\
\hline Code 9 & $\begin{array}{l}\text { Nicht beurteilbar (z. B. Krone, ausgedehnte Kari- } \\
\text { es oder große Restauration) }\end{array}$ \\
\hline Größe der betroffenen Fläche & Kriterium \\
\hline Code - & Weniger als die Hälfte der Fläche betroffen \\
\hline Code + & Mehr als die Hälfte der Fläche betroffen \\
\hline
\end{tabular}

Tabelle 3.1: Index zur Klassifizierung dentaler Erosionen im Milchgebiss nach O'Sullivan (2000). 
Neben dem O'Sullivan-Index wurde auch der BEWE-Index (Basic Erosive Wear Examination, Bartlett et al. 2008) zur Klassifizierung angewendet. Dies ermöglicht eine Vergleichbarkeit zu neueren und zukünftigen Studien. Das Bewertungssystem weist betroffenen Zähnen einen Code von 0 bis 3 zu (Tabelle 3.2).

\begin{tabular}{|c|l|}
\hline Code & \multicolumn{1}{|c|}{ Kriterium } \\
\hline 0 & Keine Erosion \\
\hline 1 & Beginnender Verlust von Oberflächenstruktur \\
\hline $2^{*}$ & $\begin{array}{l}\text { Deutliche Schädigung, Zahnhartsubstanzverlust von weniger als } 50 \% \mathrm{der} \\
\text { Zahnoberfläche }\end{array}$ \\
\hline $3^{*}$ & $\begin{array}{l}\text { Deutliche Schädigung, Zahnhartsubstanzverlust von mehr als } 50 \% \mathrm{der} \\
\text { Zahnoberfläche }\end{array}$ \\
\hline & *bei Wert 2 und 3 liegt häufig eine Dentinbeteiligung vor \\
\hline
\end{tabular}

Tabelle 3.2: BEWE-Bewertungssystem nach Bartlett et al. (2008).

Bei der Anwendung des BEWE-Index wird das zu bewertende Gebiss in Sextanten aufgeteilt. In jedem Sextanten wird nur der am schwersten von Erosion betroffene Zahn bewertet. Der kumulative Wert aller Sextanten ergibt den BEWE-Summenscore zwischen 0 und 18.

Bei der klinischen Untersuchung der Okklusal- und Inzisalflächen wurde darauf geachtet, reine Attritionen von der Bewertung auszuschließen. Das klinische Erscheinungsbild von reinen Attritionen imponiert durch plane, glänzende Schlifffacetten von Agonist und Antagonist in Ober- und Unterkiefer, die in der Protrusions- oder Laterotrusions-Bewegung exakt aufeinander führen.

\subsection{Inter-Test-Reliabilität}

Der BEWE-Index ist im direkten Vergleich zum O'Sullivan-Index ein aggregierteres Maß zur Klassifizierung von dentalen Erosionen und kann aus dem O’Sullivan-Index abgeleitet werden (Tabelle 3.3). 


\begin{tabular}{|c|c|c|c|}
\hline \multicolumn{3}{|c|}{ O'Sullivan } & BEWE \\
\hline Fläche & Schweregrad & Größe & Code \\
\hline F & 0 & + & 0 \\
\hline A-F & $1-2$ & $+/-$ & 1 \\
\hline A-F & $3-5$ & - & 2 \\
\hline A-F & $3-5$ & + & 3 \\
\hline
\end{tabular}

Tabelle 3.3: Schema zur Überführung der O'Sullivan-Codierung in die entsprechende BEWE-Codierung

Um die Inter-Test-Reliabilität zu überprüfen, wurden 656 Zähne von 33 Kindern sowohl mit dem O'Sullivan-Index als auch mit dem BEWE-Index klinisch bewertet (Tabelle 3.4). Die Klassifizierung nach O'Sullivan wurde außerdem entsprechend der in Tabelle 3.3 angegebenen Codierung in BEWE-Werte konvertiert. Danach wurden die tatsächlich erhobenen BEWE-Werte mit denen auf Basis des O'Sullivan-Index abgeleiteten BEWE-Werten statistisch verglichen. Die Übereinstimmung lag bei $98,78 \%$, die erwartete Übereinstimmung bei $61,00 \%$. Es ergab sich ein gewichteter Cohens Kappa-Wert von 0,969.

\begin{tabular}{|c|c|c|c|c|c|c|}
\cline { 2 - 7 } \multicolumn{1}{c|}{} & \multicolumn{7}{c|}{ BEWE-Untersuchung } \\
\hline \multirow{4}{*}{\begin{tabular}{c} 
Aus \\
O‘Sullivan \\
Index ab- \\
\cline { 2 - 7 }
\end{tabular}} & BEWE & 0 & 1 & 2 & 3 & gesamt \\
\cline { 2 - 7 } geleiteter & 1 & 3 & 138 & 1 & 0 & 142 \\
\cline { 2 - 7 } BEWE & 2 & 0 & 1 & 5 & 0 & 6 \\
\cline { 2 - 7 } & 3 & 0 & 0 & 0 & 16 & 16 \\
\cline { 2 - 7 } & gesamt & 492 & 142 & 6 & 16 & 656 \\
\hline
\end{tabular}

Tabelle 3.4: Inter-Test-Reliabilität

Aufgrund dieser konsistenten Ergebnisse wurden die BEWE-Werte innerhalb dieser Studie systematisch aus dem O'Sullivan-Index abgeleitet und nicht tatsächlich gemessen. Dadurch konnte die Untersuchungszeit der Kinder reduziert werden. 
3.5 Inter- und Intra-Untersucher-Reliabilität

Zur Überprüfung der Inter-Untersucher-Reliabilität wurden 53 Kinder mit insgesamt 1076 Zähnen sowohl von der Leiterin (der Untersucherin der Studie von Wiegand et al. 2006) als auch von der Untersucherin der vorliegenden Studie unabhängig voneinander mittels BEWE-Index untersucht (Tabelle 3.5). Die Übereinstimmung lag bei $95.35 \%$, die erwartete Übereinstimmung bei $70.03 \%$. Es ergab sich ein gewichteter Cohens Kappa-Wert von 0.851.

\begin{tabular}{|c|c|c|c|c|c|c|}
\hline & \multicolumn{6}{|c|}{ Christina Müller Pflanz } \\
\hline \multirow{6}{*}{$\begin{array}{l}\text { Prof. Dr. } \\
\text { Annette } \\
\text { Wiegand }\end{array}$} & BEWE & 0 & 1 & 2 & 3 & gesamt \\
\hline & 0 & 877 & 18 & 1 & 1 & 897 \\
\hline & 1 & 4 & 115 & 10 & 8 & 137 \\
\hline & 2 & 0 & 0 & 20 & 0 & 20 \\
\hline & 3 & 1 & 1 & 6 & 14 & 22 \\
\hline & gesamt & 882 & 134 & 37 & 23 & 1076 \\
\hline
\end{tabular}

Tabelle 3.5: Inter-Untersucher-Reliabilität

Zur Überprüfung der Intra-Untersucher-Reliabilität wurden 33 Kinder mit 656 Zähnen jeweils an zwei verschiedenen Zeitpunkten von der Untersucherin der vorliegenden Studie mittels BEWE-Index untersucht (Tabelle 3.6). Die Übereinstimmung lag bei $94.82 \%$, die erwartete Übereinstimmung bei $60.68 \%$. Es ergab sich ein gewichteter Cohens Kappa-Wert von 0,753.

\begin{tabular}{|c|c|c|c|c|c|c|}
\hline & \multicolumn{6}{|c|}{ 1. Untersuchung } \\
\hline \multirow{6}{*}{$\begin{array}{l}\text { 2. Unter- } \\
\text { suchung }\end{array}$} & BEWE & 0 & 1 & 2 & 3 & gesamt \\
\hline & 0 & 475 & 13 & 0 & 0 & 488 \\
\hline & 1 & 17 & 127 & 2 & 0 & 146 \\
\hline & 2 & 0 & 2 & 4 & 0 & 6 \\
\hline & 3 & 0 & 0 & 0 & 16 & 16 \\
\hline & gesamt & 492 & 142 & 6 & 16 & 656 \\
\hline
\end{tabular}

Tabelle 3.6: Intra-Untersucher-Reliabilität 


\subsection{Anamnesebogen}

Der von den Erziehungsberechtigten auszufüllende Anamnesebogen orientierte sich inhaltlich an den von Shaw und O'Sullivan (2000) verfassten “UK National Clinical Guidelines in Paediatric Dentistry" und an dem Anamnesebogen der Studie von Wiegand et al. (2006). In drei aufeinanderfolgenden Abschnitten wurden Allgemeinerkrankungen, Medikation, Ernährungsgewohnheiten und Mundhygienemaßnahmen erfragt, wobei besonders die in der Fachliteratur diskutierten Risikofaktoren für Erosionen berücksichtigt wurden (Abbildungen 7.1 und 7.2 im Anhang).

\subsection{Kindergarten-Fragebogen}

Die Erzieher/innen wurden gebeten, Angaben zu Ernährung und Zahnpflege der Kinder in ihrer Einrichtung zu machen. Zu diesem Zweck wurden die Aspekte des Anamnesebogens, welche in einem Kindergarten von Bedeutung sind, angepasst und zu einem Fragebogen zusammengefasst (Abbildungen 7.3 und $7.4 \mathrm{im}$ Anhang).

\subsection{Statistische Auswertung}

Die statistische Auswertung wurde in Zusammenarbeit mit Herrn PD Dr. Jung, Zentrale Serviceeinheit Medizinische Biometrie und Statistische Bioinformatik, durchgeführt. Die erhobenen Daten wurden mit Methoden der deskriptiven und induktiven Statistik ausgewertet.

Für die statistische Analyse der epidemiologischen Daten zur Prävalenz fanden unterschiedliche Testverfahren Anwendung. Tabelle 3.7 gibt einen Überblick über das Verfahren in Abhängigkeit der jeweiligen Forschungsfrage. 


\begin{tabular}{|c|c|}
\hline Untersuchter Zusammenhang & Statistisches Verfahren \\
\hline Abhängigkeit der Prävalenz vom Alter & Mann-Whitney-U-Test \\
\hline $\begin{array}{c}\text { Vergleich der Prävalenz zwischen Ober- und } \\
\text { Unterkiefer }\end{array}$ & t-Test \\
\hline $\begin{array}{c}\text { Abhängigkeit des Schweregrads der Erosionen } \\
\text { (BEWE-Summenscore) vom Alter }\end{array}$ & $\begin{array}{c}\text { Spearmans Rangkorrelations- } \\
\text { koeffizient }\end{array}$ \\
\hline $\begin{array}{l}\text { Vergleich des Schweregrads der Erosionen (BE- } \\
\text { WE-Summenscore) zwischen den Altersklassen } \\
\text { und Jungen und Mädchen }\end{array}$ & Mann-Whitney-U-Test \\
\hline
\end{tabular}

Tabelle 3.7: Statistische Verfahren.

Für die Analyse des Einflusses potentieller Risikofaktoren wurden zwei verschiedene Regressionsmodelle geschätzt. Der Einfluss potentieller Faktoren auf das Vorliegen dentaler Erosionen wurde mit logistischen Regressionen, die ordinale Messgröße BEWE-Summenscore mit linearen Regressionen untersucht (Tabelle 3.8).

\begin{tabular}{|c|c|c|}
\hline Messgröße & Skalierung & Statistisches Verfahren \\
\hline $\begin{array}{c}\text { Vorliegen dentaler } \\
\text { Erosionen }\end{array}$ & $\begin{array}{c}0-\text { Kind hat keine Erosion } \\
1-\text { Kind hat Erosion }\end{array}$ & Logistische Regression \\
\hline BEWE-Summenscore & $0-18$ & Lineare Regression \\
\hline
\end{tabular}

Tabelle 3.8: Risikofaktoren: Messgrößen und statistische Verfahren.

Bei beiden Regressionen wurde zur Bestimmung des finalen Modells ein schrittweises Auswahlverfahren angewendet. Einige Variablen konnten aufgrund zu weniger Beobachtungen nicht in die Analyse aufgenommen werden. Dies betraf die Faktoren „Diabetes“, „Reflux“ und „Erbrechen“ aus dem Teil „Allgemeinerkrankungen“ des Anamnesebogens. Darüber hinaus wurden Antworten zu Unterfragen, wie z. B. „Dauer der Stillzeit“ oder „Dauer der Medikation“, aufgrund zu weniger Beobachtungen nicht untersucht (Abbildungen 7.1 und 7.2 im Anhang). Die restlichen Faktoren, die als unabhängige Variablen der schrittweisen Elimination zugeführt wurden, sind in Tabelle 3.9 aufgeführt. Zur Auswertung der Fragebögen aus den Kindergärten wurden zusätzlich die Antworten 
zu Ernährung und Mundhygiene aus den Kindergärten der schrittweisen Elimination zugeführt.

\begin{tabular}{|c|c|c|}
\hline & Potentielle Risikofaktoren & Einteilung \\
\hline \multirow{5}{*}{ 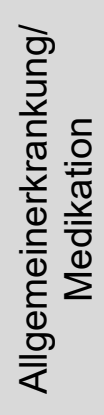 } & Lungenerkrankung/Asthma & $0-$ nein, $1-$ ja \\
\hline & Therapie mittels Inhalationssprays & $0-$ nein, $1-$ ja \\
\hline & Allgemeinerkrankung & $0-$ nein, $1-$ ja \\
\hline & Regelmäßige Medikamenteneinnahme & $0-$ nein, $1-$ ja \\
\hline & Regelmäßige Fluorideinnahme & $0-$ nein, $1-$ ja \\
\hline \multirow{19}{*}{ 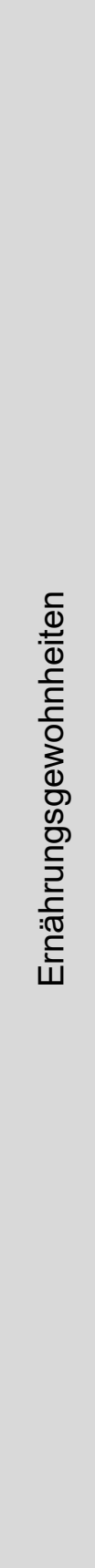 } & Gestillt & $0-$ nein, $1-$ ja \\
\hline & Fläschchen & $0-$ nein, $1-$ ja \\
\hline & Fläschchen zum Einschlafen & $0-$ nein, $1-$ ja \\
\hline & \multicolumn{2}{|c|}{ Frequenz des Konsums von: } \\
\hline & Fruchtsaft pur & \multirow{10}{*}{$\begin{array}{c}1 \text { - nie/selten, } \\
2-\text { wöchentlich, } \\
3-\text { täglich, } \\
4-2 \text { bis } 3 \text { mal täglich, } \\
5 \text { - häufiger }\end{array}$} \\
\hline & Fruchtsaft verdünnt & \\
\hline & Zitronentee & \\
\hline & Limo/Cola & \\
\hline & Sprudel & \\
\hline & Stilles Wasser & \\
\hline & Milch & \\
\hline & Zitrusfrüchte & \\
\hline & Süßigkeiten & \\
\hline & Zitronensprudel & \\
\hline & \multicolumn{2}{|c|}{ Häufigkeit der Getränkeaufnahme durch: } \\
\hline & Becher & \multirow{4}{*}{$\begin{array}{c}1 \text { - nie, } 2 \text { - selten, } 3 \text { - manchmal, } \\
4 \text { - oft, } 5 \text {-immer }\end{array}$} \\
\hline & Strohhalm & \\
\hline & Trinklernbecher & \\
\hline & Flasche & \\
\hline
\end{tabular}




\begin{tabular}{|c|c|c|}
\hline \multirow{7}{*}{ 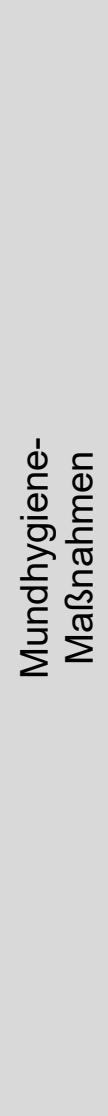 } & \multicolumn{2}{|c|}{ Zähneputzen: } \\
\hline & Wer & $\begin{array}{c}1 \text { - Kind, } 2 \text { - Eltern/Erzieher, } \\
3 \text { - erst Kind, dann El- } \\
\text { tern/Erzieher }\end{array}$ \\
\hline & Wie oft & Wie oft täglich: $1-3$ \\
\hline & Wie lange & Minuten: $1-3$ \\
\hline & Art der Zahnbürste & $\begin{array}{c}1 \text { - Handzahnbürste, } 2 \text { - Hand- } \\
\text { und elektrische Zahnbürste, } \\
\text { 3 - elektrische Zahnbürste }\end{array}$ \\
\hline & Art der Zahnpasta & $\begin{array}{c}0 \text { - fluoridierte Kinder- oder } \\
\text { Erwachsenenzahnpasta } \\
1 \text { - nicht-fluoridierte Kinder- oder } \\
\text { Erwachsenenzahnpasta }\end{array}$ \\
\hline & Speisen/Getränke nach Zähr & $0-$ nein, $1-$ ja \\
\hline
\end{tabular}

Tabelle 3.9: Potentielle Risikofaktoren, die als Regressoren verwendet wurden.

Die Auswertungen erfolgten mit der Software STATA 12.1 (StataCorp LP, TX, USA). 


\section{Ergebnisse}

Aus 27 Kindergärten (Abbildung 4.1) nahmen 777 Kinder (371 Mädchen, 406 Jungen) im Alter von 3 bis 7 Jahren an der klinischen Untersuchung teil. Es wurden insgesamt 15523 Zähne (15052 Milchzähne, 471 bleibende Zähne) untersucht. 138 der untersuchten Kinder hatten mindestens einen bleibenden Zahn. Keiner der bleibenden Zähne zeigte Erosionen. Von den untersuchten Milchzähnen waren 161 Front- und Eckzähne und 119 Molaren fehlend oder aufgrund kariöser Läsionen, großer Restaurationen oder prothetischer Versorgungen nicht beurteilbar. Die nicht beurteilbaren Zähne wurden von der Auswertung ausgeschlossen.

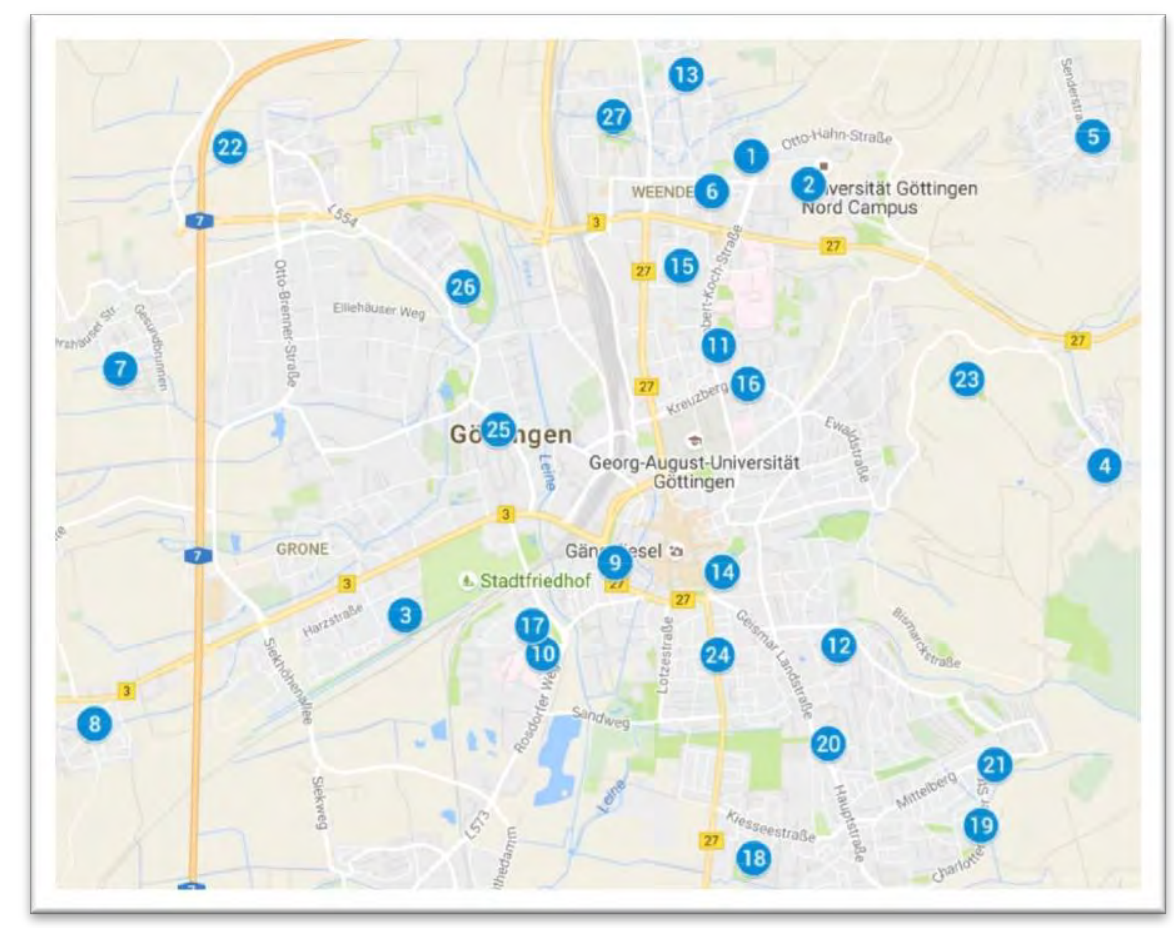

Abbildung 4.1: Übersichtskarte und Verteilungsmuster der Kindergärten, die an der vorliegenden Studie teilgenommen haben.

\subsection{Ergebnisse der klinischen Untersuchung}

Da keiner der bleibenden Zähne Erosionen aufwies, beschränken sich die Abbildungen zur besseren grafischen Darstellung auf die Milchzähne. Des Weiteren waren nur zwei der untersuchten Kinder im Alter von 7 Jahren. Diese beiden Beobachtungen wurden daher mit den 152 Beobachtungen für die 6Jährigen zusammengefasst. 


\subsubsection{Prävalenz der Erosionen in Abhängigkeit von Alter und Geschlecht}

Von den 777 untersuchten Kindern wiesen 353 (45,4\%) mindestens einen Zahn mit Erosionen auf. Es war ein signifikanter Anstieg des Anteils der von Erosionen betroffenen Kinder mit zunehmendem Alter zu erkennen: 3-Jährige: 14,2\%; 4-Jährige: 32,9\%; 5-Jährige: 58,8\%; 6-7-Jährige: 71,4\% (Tabelle 4.1, p<0,01).

Die logistische Regression zeigte ebenfalls, dass ältere Kinder signifikant häufiger von Erosionen betroffen waren $(p<0,001)$. Außerdem zeigten Jungen mit 50\% (203 von 406) eine signifikant höhere Prävalenz als Mädchen mit 40,4\% (150 von $371, p=0,008)$.

\begin{tabular}{|c|c|c|c|c|c|}
\hline Alter & $\begin{array}{c}\text { Anzahl der } \\
\text { untersuch- } \\
\text { ten Kinder } \\
(\mathbf{N})\end{array}$ & $\begin{array}{c}\text { Kinder } \\
\text { mit } \\
\text { Erosionen } \\
(\mathrm{N})\end{array}$ & $\begin{array}{c}\mathbf{1 - 5} \text { Zähne } \\
\text { mit } \\
\text { Erosionen } \\
(\mathrm{N})\end{array}$ & $\begin{array}{c}\mathbf{6 - 1 0} \text { Zähne } \\
\text { mit } \\
\text { Erosionen } \\
(\mathrm{N})\end{array}$ & $\begin{array}{c}\text { Mehr als 10 } \\
\text { Zähne mit } \\
\text { Erosionen } \\
(\mathrm{N})\end{array}$ \\
\hline $\mathbf{3}$ & 134 & 19 & 10 & 7 & 2 \\
\hline $\mathbf{4}$ & 246 & 81 & 41 & 18 & 22 \\
\hline $\mathbf{5}$ & 243 & 143 & 55 & 45 & 43 \\
\hline $\mathbf{6 - 7}$ & 154 & 110 & 24 & 48 & 38 \\
\hline gesamt & 777 & $\mathbf{3 5 3}$ & $\mathbf{1 3 0}$ & $\mathbf{1 1 8}$ & $\mathbf{1 0 5}$ \\
\hline & & $\mathbf{4 5 , 4 \%}$ & $\mathbf{1 6 , 7 \%}$ & $\mathbf{1 5 , 2} \%$ & $\mathbf{1 3 , 5 \%}$ \\
\hline
\end{tabular}

Tabelle 4.1: Prävalenz der Erosionen nach Altersgruppen, N = Anzahl der Kinder

4.1.2 Prävalenz der Erosionen in Ober- und Unterkiefer und Front- und Seitenzahnbereich

Die steigende Prävalenz von Erosionen mit zunehmendem Alter war im Oberwie im Unterkiefer zu beobachten (Tabelle 4.2). Es zeigte sich, dass in jeder Altersklasse der Oberkiefer signifikant häufiger betroffen war als der Unterkiefer $(p<0,001)$. 


\begin{tabular}{|c|c|c|}
\hline Alter & Oberkiefer & Unterkiefer \\
\hline $\mathbf{3}$ & $14,2 \%$ & $7,5 \%$ \\
\hline $\mathbf{4}$ & $30,9 \%$ & $17,5 \%$ \\
\hline $\mathbf{5}$ & $58,4 \%$ & $34,2 \%$ \\
\hline $\mathbf{6 - 7}$ & $70,1 \%$ & $53,9 \%$ \\
\hline
\end{tabular}

Tabelle 4.2: Anteil der Kinder mit Erosionen im Ober- und Unterkiefer je Altersgruppe.

Die größere Häufigkeit von Erosionen im Oberkiefer war im Besonderen für die Front- und Eckzähne zu beobachten, die im Oberkiefer meist mehr als doppelt so häufig Erosionen aufwiesen (Abbildung 4.2).

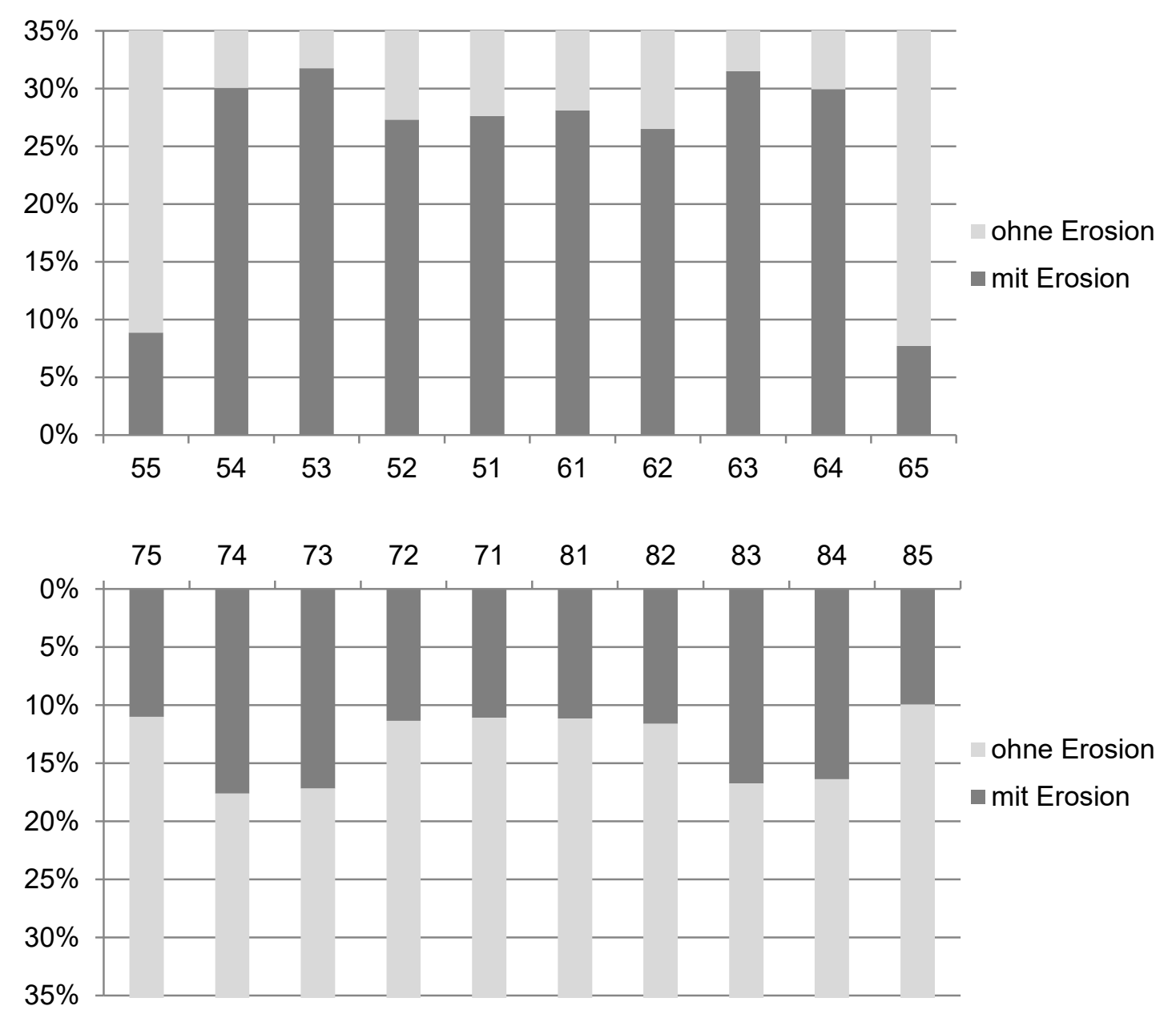

Abbildung 4.2: Prävalenz dentaler Erosionen bei allen Zähnen des Milchgebisses.

Von den 353 von Erosionen betroffenen Kindern wiesen 18,7\% ausschließlich Erosionen im Front- und Eckzahnbereich auf. Eine ausschließliche Beteiligung der Molaren war in 9,9\% der Fälle zu beobachten. Die große Mehrheit der Kin- 
der $(71,4 \%)$ zeigte erosive Läsionen sowohl an Front- und Eckzähnen, als auch an den Molaren.

4.1.3 Schweregrad und Lokalisation der Erosionen anhand des O'SullivanIndex

Die Tabellen 4.4. und 4.5 geben die Lokalisation, den Schweregrad und die Größe der von Erosionen betroffenen Zahnflächen bei Front- und Eckzähnen und den Molaren anhand des O`Sullivan-Index wieder.

Sowohl bei den Front- und Eckzähnen als auch bei den Molaren wies der Großteil der betroffenen Flächen einen Schweregrad von 2 (Kontinuitätsverlust der Schmelzoberfläche) bis 3 (Kontinuitätsverlust bis zur Schmelz-Dentin-Grenze) auf. Dies traf mit 87,7\% auf die Front- und Eckzähne und mit 91,9\% auf die Molaren zu. Erosionen, die durch Schmelz und Dentinverlust mit Pulpafreilegung und einen Schweregrad von 5 charakterisiert waren, kamen sehr selten und nur bei den Front- und Eckzähnen vor (<1\%).

Bei den Front- und Eckzähnen waren Erosionen am häufigsten auf die Inzisalkante $(45,5 \%)$ und am zweithäufigsten auf die Inzisalkante in Verbindung mit der palatinalen oder lingualen Zahnfläche (31,5\%) beschränkt. Bei den Molaren waren die Okklusalfächen (74,2\%) mit Abstand am häufigsten betroffen. Bei $13,5 \%$ waren multiple Flächen betroffen.

\begin{tabular}{|c|c|c|c|c|c|c|c|c|c|c|c|c|c|c|c|}
\hline \multirow{2}{*}{ S } & \multirow{2}{*}{$\mathrm{N}$} & \multicolumn{12}{|c|}{ Betroffene Flächen von Front- und Eckzähnen [\%] } & \multicolumn{2}{|c|}{$\begin{array}{c}\text { gesamt } \\
\text { [\%] }\end{array}$} \\
\hline & & $A+$ & A- & $\mathrm{B}+$ & B- & $\mathrm{C}+$ & C- & D+ & D- & $\mathrm{E}+$ & E- & $\mathrm{F}+$ & F- & “+” & “-“ \\
\hline 1 & 158 & 0,0 & 0,0 & 5,1 & 0,0 & 7,6 & 0,6 & 0,6 & 0,0 & 26,6 & 1,3 & 55,7 & 2,5 & 95,6 & 4,4 \\
\hline 2 & 1147 & 0,0 & 0,1 & 1,0 & 0,1 & 26,5 & 29,5 & 1,0 & 1,5 & 10,5 & 15,4 & 9,5 & 5,0 & 48,5 & 51,5 \\
\hline 3 & 513 & 0,0 & 0,0 & 0,8 & 0,4 & 15,4 & 23,6 & 0,6 & 3,7 & 14,4 & 26,5 & 2,1 & 12,5 & 33,3 & 66,7 \\
\hline 4 & 61 & 0,0 & 0,0 & 0,0 & 0,0 & 4,9 & 4,9 & 3,3 & 4,9 & 26,2 & 29,5 & 4,9 & 21,3 & 39,3 & 60,7 \\
\hline 5 & 14 & 0,0 & 0,0 & 0,0 & 0,0 & 0,0 & 0,0 & 0,0 & 0,0 & 21,4 & 50,0 & 0,0 & 28,6 & 21,4 & 78,6 \\
\hline & 1893 & 0,0 & 0,1 & 1,2 & 0,2 & 21,0 & 24,5 & 0,9 & 2,1 & 13,5 & 18,0 & 11,1 & 7,5 & & \\
\hline
\end{tabular}

Tabelle 4.3: $\mathrm{S}=$ Schwergrad nach O'Sullivan (2000), N = Anzahl der von Erosionen betroffenen Front- und Eckzähne, A+ bis F- in \% = betroffene Zahnflächen, ,,+“ und „,", in \% = Zahnflächen, die mehr (+) bzw. weniger (-) als $50 \%$ von Erosionen betroffen waren. 


\begin{tabular}{|c|c|c|c|c|c|c|c|c|c|c|c|c|c|c|c|}
\hline \multirow{2}{*}{ S } & \multirow{2}{*}{ N } & \multicolumn{12}{|c|}{ Betroffene Flächen von Molaren [\%] } & \multicolumn{2}{|c|}{$\begin{array}{c}\text { gesamt } \\
{[\%]}\end{array}$} \\
\hline & & $A+$ & A- & $\mathrm{B}+$ & B- & $\mathrm{C}+$ & C- & D+ & D- & $E+$ & E- & $\mathrm{F}+$ & F- & “+” & “-“ \\
\hline 1 & 64 & 0,0 & 0,0 & 4,7 & 0,0 & 32,8 & 14,1 & 1,6 & 0,0 & 10,9 & 0,0 & 34,4 & 1,6 & 84,4 & 15,6 \\
\hline 2 & 771 & 0,1 & 0,1 & 1,0 & 0,6 & 8,8 & 71,1 & 2,6 & 1,6 & 1,8 & 2,7 & 7,1 & 2,3 & 21,5 & 78,5 \\
\hline 3 & 144 & 0,0 & 0,0 & 0,7 & 0,7 & 4,2 & 52,8 & 1,4 & 6,9 & 0,7 & 7,6 & 6,3 & 18,8 & 13,2 & 86,8 \\
\hline 4 & 17 & 0,0 & 0,0 & 0,0 & 0,0 & 5,9 & 58,8 & 0,0 & 11,8 & 0,0 & 5,9 & 0,0 & 17,6 & 5,9 & 94,1 \\
\hline 5 & 0 & 0,0 & 0,0 & 0,0 & 0,0 & 0,0 & 0,0 & 0,0 & 0,0 & 0,0 & 0,0 & 0,0 & 0,0 & 0,0 & 0,0 \\
\hline & 996 & 0,1 & 0,1 & 1,2 & 0,6 & 9,6 & 64,6 & 2,3 & 2,4 & 2,2 & 3,3 & 8,6 & 4,9 & & \\
\hline
\end{tabular}

Tabelle 4.4: $\mathrm{S}=$ Schwergrad nach O'Sullivan (2000), $\mathrm{N}=$ Anzahl der von Erosionen betroffenen Molaren, A+ bis F- in \% = betroffene Zahnflächen, ,"“ und „,-,, in \% = Zahnflächen, die mehr (+) bzw. weniger (-) als $50 \%$ von Erosionen betroffen waren.

4.1.4 Schweregrad und Lokalisation der Erosionen anhand des BEWE-Index In Abbildung 4.3 ist der Schweregrad und die Lokalisation der erosiven Läsionen anhand des BEWE-Index dargestellt.

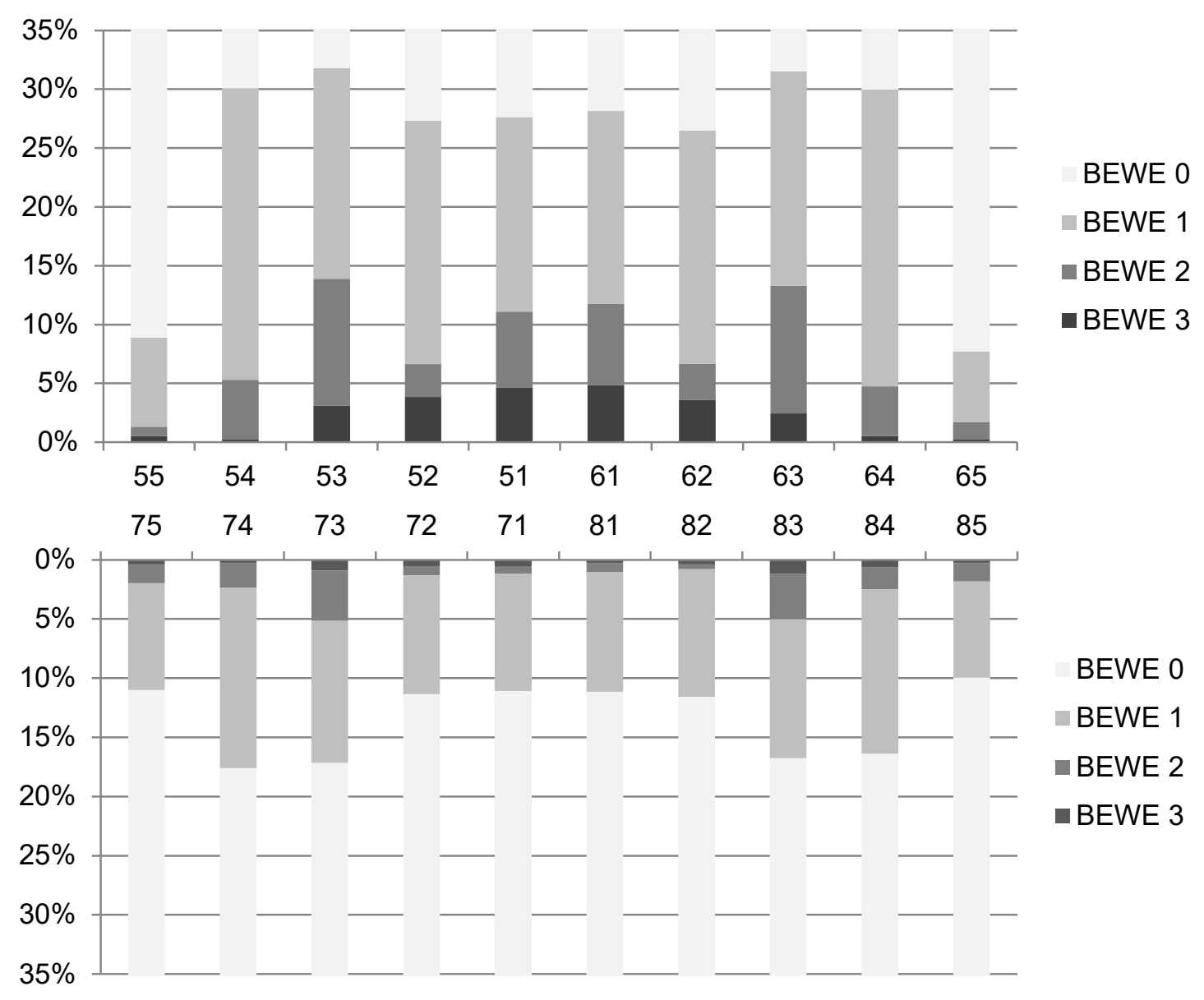

Abbildung 4.3: Verteilung der Erosionen entsprechend dem BEWE-Index bei allen Zähnen des Milchgebisses [\%]. 
Die Erosionen im Oberkiefer zeigten insgesamt einen höheren Schweregrad als die Erosionen im Unterkiefer. Dies war für jede Altersgruppe zu beobachten (Abbildung 4.4 und Abbildung 4.5).

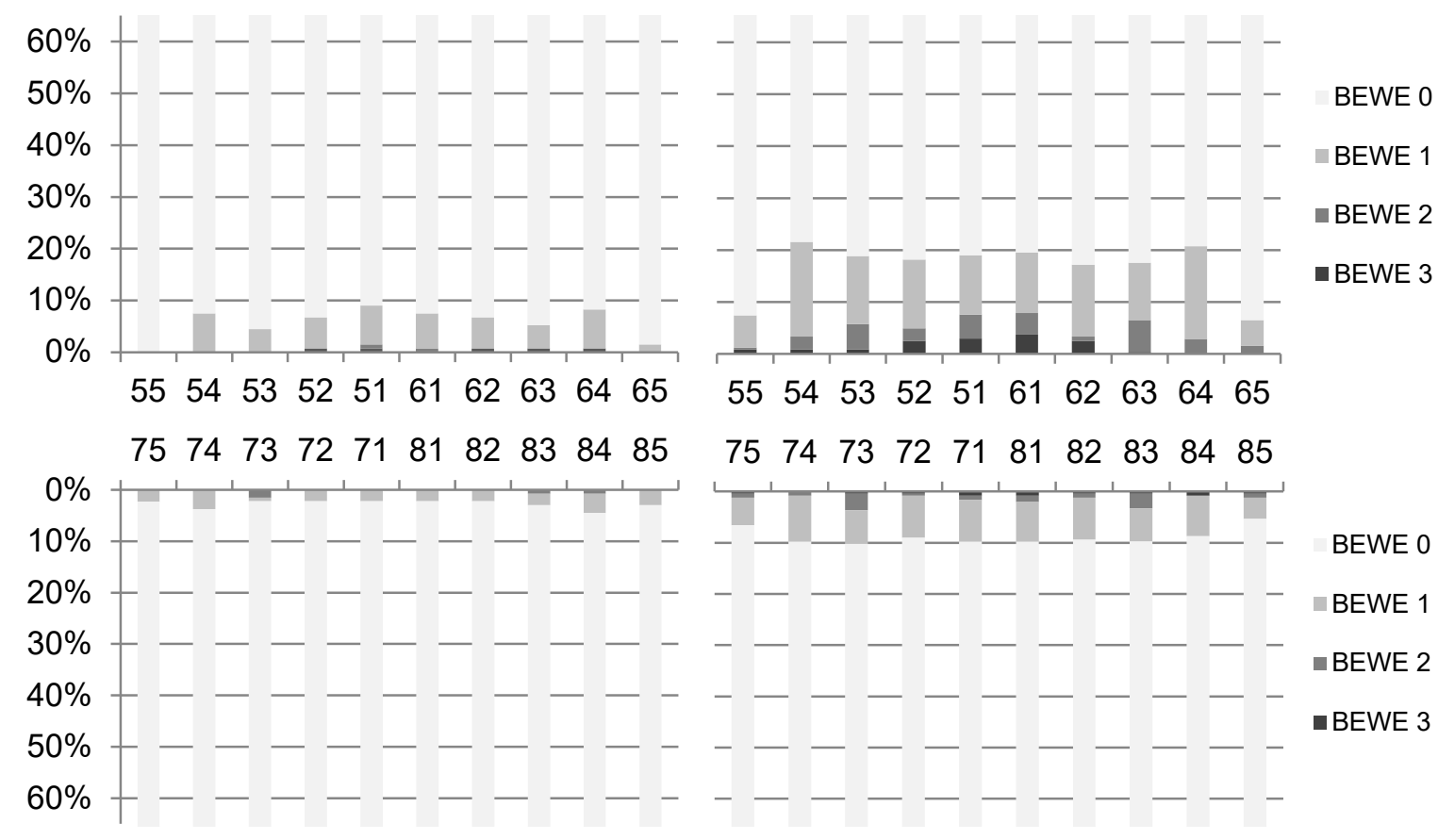

Abbildung 4.4: Verteilung der Erosionen in Ober- und Unterkiefer bei 3-Jährigen (links) und 4-Jährigen (rechts) [\%].

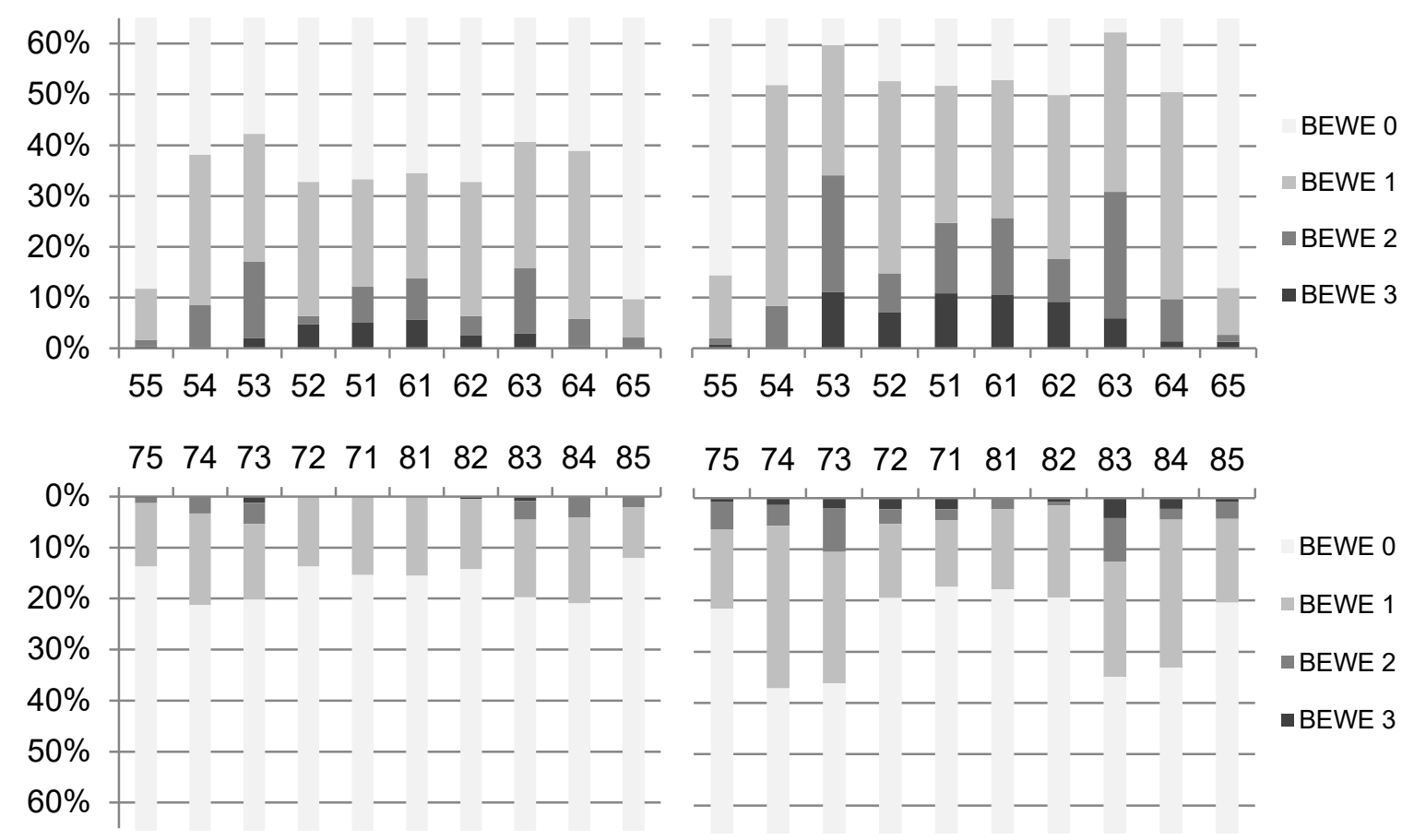

Abbildung 4.5: Verteilung der Erosionen in Ober- und Unterkiefer bei 5-Jährigen (links) und 6-7-Jährigen (rechts) [\%]. 
Tabelle 3.3 zeigt die Verteilung über die verschiedenen Risikoklassen in Abhängigkeit vom Alter. Etwas mehr als 5\% der untersuchten Kinder ließen sich der mittleren und hohen Risikoklasse zuordnen.

\begin{tabular}{|c|c|c|c|c|c|c|}
\hline Risiko & $\begin{array}{c}\text { BEWE- } \\
\text { Summen- } \\
\text { score }\end{array}$ & $\%$ & 3-Jährige & 4-Jährige & 5-Jährige & 6-7-Jährige \\
\hline Kein & $0-2$ & 66,4 & $92,5 \%$ & $78,5 \%$ & $58,4 \%$ & $36,8 \%$ \\
\hline Niedrig & $3-8$ & 28,1 & $7,5 \%$ & $17,5 \%$ & $35,8 \%$ & $50,7 \%$ \\
\hline Mittel & $9-13$ & 5,0 & $0,0 \%$ & $3,7 \%$ & $5,8 \%$ & $10,5 \%$ \\
\hline Hoch & $14-18$ & 0,5 & $0,0 \%$ & $0,4 \%$ & $0,0 \%$ & $2,0 \%$ \\
\hline
\end{tabular}

Tabelle 4.5: Verteilung der untersuchten Kinder entsprechend der Risiko-Klassifizierung nach dem BEWE-Summenscore (Klassifikation in Anlehnung an Bartlett et al. 2008).

\subsection{Risikofaktoren}

Die logistische Regression zeigte, dass in der Gesamtstichprobe ältere Kinder (Odds Ratio: 2,550, $p<0,001$ ) und Jungen (Odds Ratio: 1,675, $p=0,002$ ) signifikant häufiger von Erosionen betroffenen waren. Die Zufuhr von Speisen oder Getränken nach dem abendlichen Zähneputzen war signifikant häufiger mit Erosionen assoziiert (Odds Ratio: 1,444, p=0,043).

Die multilineare Regression zeigte, dass in der Gesamtstichprobe ältere Kinder $(p<0,001)$ und Jungen $(p=0,008)$ auch signifikant schwerere Erosionen aufwiesen. Dies spiegelte sich einerseits in einer signifikanten Korrelation zwischen dem BEWE-Summenscore und dem Alter $(p<0,001)$, andererseits in einem signifikant höheren BEWE-Summenscore für Jungen wider $(p=0,013)$. Außerdem war der häufigere Konsum von Limo und Cola signifikant mit schwereren Erosionen assoziiert $(p=0,001)$. Dies verdeutlichte auch die signifikante positive Korrelation des BEWE-Summenscores mit der Häufigkeit des Konsums von Limo und Cola $(p=0,005)$.

Die multilineare Regression für die Teilstichprobe der von Erosionen betroffenen Kinder bestätigte den signifikanten Zusammenhang zwischen dem Alter und der Schwere der Erosionen $(p<0,001)$, sowie die Assoziation häufigeren Konsums von Limo und Cola mit schwereren Erosionen $(p=0,013)$. 


\subsubsection{Rolle der Kindergärten}

Zur Untersuchung des Einflusses der Ernährung und der angebotenen Mundhygiene in den Kindergärten wurde das multilineare Regressionsmodell inklusive der Variablen zu den Kindergartenangaben geschätzt. Die Regressionsergebnisse bestätigten die in 4.2 identifizierten Risikofaktoren. Keine der kindergartenspezifischen Variablen zeigte einen signifikanten Einfluss. 


\section{Diskussion}

\subsection{Diskussion der Methode}

\subsubsection{Probandengut}

Aufgrund des gleichmäßigen Verteilungsmusters der teilnehmenden Kindergärten über die verschiedenen Stadtteile von Göttingen kann davon ausgegangen werden, dass die untersuchte Stichprobe einem repräsentativen Bevölkerungsquerschnitt entspricht (Abbildung 4.1). Die Stichprobe zeichnet sich durch ein ausgewogenes Geschlechterverhältnis aus.

\section{1 .2 Indizes}

Zur klinischen Untersuchung wurde der O‘Sullivan-Index (O'Sullivan et al. 2000) herangezogen. Dieser Index wird in vielen weiteren epidemiologischen Untersuchungen bei Kindern und Jugendlichen verwendet (Caglar et al. 2005, Peres et al. 2005, Wiegand et al. 2006, Correr et al. 2009, Kumar et al. 2013, Tao et al. 2015, Kirthiga et al. 2015). Durch die Anwendung des O'Sullivan-Index ist es möglich, die erhobenen Daten der vorliegenden Studie direkt mit der vor 10 Jahren durchgeführten Untersuchung zur Prävalenz von dentalen Erosionen bei Göttinger Kindergartenkindern zu vergleichen (Wiegand et al. 2006). Der O'Sullivan-Index ist auf jeden Zahn anwendbar und erlaubt aufgrund der zahlreichen Abstufungen eine sehr differenzierte Befundaufnahme.

Um die Untersuchungszeit für jedes Kind so kurz wie möglich zu gestalten, wurden die klinisch gemessenen O'Sullivan-Werte in systematisch abgeleitete BEWE-Werte konvertiert. Die Inter-Test-Reliabilität entspricht laut Greve und Wentura (1997) oder Landis und Koch (1977) einer "guten bis ausgezeichneten“ bzw. „(fast) vollkommenen Übereinstimmung“ (gewichteter Cohens Kappa von 0,969). Der BEWE-Index findet in vielen weiteren Studien Anwendung (Kaczmarek et al. 2012, Manaf et al. 2012, Mantonanaki et al 2013, Zhang et al. 2014, Vered et al. 2014, Chu et al. 2015, Alves et al. 2015, MullerBolla et al. 2015, Alvarez Loureiro et al. 2015). 


\subsubsection{Klinische Untersuchung}

In der vorliegenden Studie wurden alle beurteilbaren Zähne und Zahnflächen bewertet. Einige Studien beschränken sich auf die Untersuchung sogenannter Markerzähne, z. B. der Oberkiefer-Milchfrontzähne oder der Milchmolaren (AlMalik et al. 2002, Al-Majed et al. 2002, Murakami et al. 2011). Andere Studien bewerten nur die Labial- und Palatinalflächen der Oberkiefer-Milchzähne (Harding et al. 2003) oder schließen die inzisalen Zahnflächen von der Beurteilung aus (Millward et al. 1994, Luo et al. 2005, Murakami et al. 2011). Im Gegensatz dazu können durch die Untersuchung aller Zähne und Zahnflächen Erkenntnisse über die Verteilung und den Schweregrad der Erosionen sowie deren Lokalisation im gesamten Gebiss gewonnen werden.

Zur Überprüfung der Güte der Befunderhebung dieser Studie wurde die InterUntersucher-Reliabilität zwischen der Untersucherin und der Leiterin dieser Studie (Untersucherin von Wiegand et al. 2006) bestimmt. Der resultierende gewichtete Cohens Kappa-Wert entspricht mit 0,851 einer „guten bis ausgezeichneten“ bzw. „(fast) vollkommenen Übereinstimmung“ (Greve und Wentura 1997, Landis und Koch 1977). Darüber hinaus wurde die Intra-UntersucherReliabilität zur Überprüfung der internen Konsistenz bestimmt. Der resultierende gewichtete Cohens Kappa-Wert von 0,753 entspricht einer „guten bis ausgezeichneten“ bzw. „beachtlichen Übereinstimmung“ (Greve und Wentura 1997, Landis und Koch 1977).

\subsubsection{Anamnesebogen und Kindergarten-Fragebogen}

Der von den Erziehungsberechtigten auszufüllende Anamnesebogen erfasste die Allgemeinerkrankungen, Medikation, Mundhygiene- und Ernährungsgewohnheiten der untersuchten Kinder. Seine Struktur orientierte sich an den "UK National Clinical Guidelines in Paediatric Dentistry" (Shaw und O'Sullivan 2000) und an dem von Wiegand et al. (2006) entwickelten Anamnesebogen.

In zahlreichen anderen Untersuchungen zur Prävalenz von dentalen Erosionen bei Kindern bis 7 Jahre wird die Anamnese zur Risikofaktoranalyse ebenfalls mit einem Fragebogen erhoben (Al-Malik et al. 2002, Al-Majed et al. 2002, Harding et al. 2003, Luo et al. 2005, Wiegand et al. 2006, Mantonanaki et al. 
2011, Murakami et al. 2011, Gatou und Mamai-Homata 2012, Moimaz et al. 2013, Tao et al. 2015).

Aus psychologischen Motiven heraus könnten die Eltern auf Fragen zu Ernährung und Mundhygiene nicht immer wahrheitsgemäß antworten. Beispielsweise könnten Eltern von Kindern mit schweren Erosionen aus einem Schamgefühl heraus positivere Antworten, z. B. den erosiven Getränkekonsum betreffend, geben. In den Antworten der Eltern mit einem hohen Bildungsniveau könnte sich eher das Wissen um das korrekte Mundhygieneverhalten oder eine gesunde Ernährung widerspiegeln als das, was tatsächlich im Altag praktiziert wird. So könnte die Risikofaktoranalyse allgemein einer Verzerrung unterliegen.

Um eine breite Zustimmung der Befragung innerhalb dieser Studie zu erreichen, wurde auf einen sozioökonomischen Fragen-Komplex, wie beispielsweise Fragen zum Bildungsgrad der Eltern und zum Familieneinkommen, verzichtet. Andere Untersuchungen können einen im Vorfeld vermuteten Zusammenhang zwischen der Zugehörigkeit zu einer bestimmten gesellschaftlichen Schicht und dem Vorkommen von dentalen Erosionen bestätigen, kommen dabei aber zu widersprüchlichen Ergebnissen (Millward et al. 1994, Harding et al. 2003, Luo et al. 2005, Mantonanaki et al. 2013, Tao et al. 2015, siehe 5.2.6).

Der von den Kindergärten auszufüllende Fragebogen erfasste die Ernährung und die Mundhygiene des jeweiligen Kindergartens. Die Fragen entsprachen den Fragen des Anamnesebogens für die Eltern, insofern sie für einen Kindergarten relevant waren. Die Kindergärten könnten unterschiedliche Betreuungskonzepte verfolgen, was sich in Unterschieden in der Ernährung und der angebotenen Mundhygiene widerspiegeln und einen Einfluss auf die Mundgesundheit der Kinder haben könnte. Auch die Kindergärten könnten nicht wahrheitsgemäß auf die Fragebögen geantwortet haben. Eventuell könnten angebotene Betreuungskonzepte, wie z. B. das tägliche Zähneputzen, aus Personal- oder Zeitmangel nicht so umgesetzt werden, wie im Fragebogen angegeben. 


\subsection{Diskussion der Ergebnisse}

\subsubsection{Ergebnisse der klinischen Untersuchung}

\subsubsection{Prävalenz und Schwere der Erosionen}

In der vorliegenden Untersuchung wiesen $45,4 \%$ der untersuchten 3 - bis 7 jährigen Kinder mindestens einen Zahn mit dentalen Erosionen auf. 63,2\% der betroffenen Kinder zeigten an mindestens 6 Zähnen einen erosiven Zahnhartsubstanzverlust Bei 59,5\% erstreckte sich dieser bis in das Dentin. Wiegand et al. (2006) stellten vor 10 Jahren bei Göttinger Kindergartenkindern eine Prävalenz von $32 \%$ fest. $68,2 \%$ der Betroffenen zeigten an bis zu 5 Zähnen Erosionen. Bei $41,2 \%$ dehnten sich diese bis in das Dentin aus.

Göttinger Kindergartenkinder waren in der aktuellen Studie häufiger, schwerer und an mehr Zähnen von Erosionen betroffen als vor 10 Jahren. Andere Studien weisen ebenfalls darauf hin, dass die Prävalenz für Erosionen bei Kindern zugenommen hat (Nunn et al. 2003, Jaeggi und Lussi 2014). Der Anstieg der Prävalenz bei Göttinger Kindergartenkindern innerhalb der letzten Dekade könnte in Veränderungen von Ernährungsgewohnheiten, wie z. B. einer Zunahme des Konsums von Softdrinks, begründet sein. In einem neueren Überblick von Studien zur Prävalenz, Inzidenz und Verteilung von Erosionen kommen Jaeggi und Lussi (2014) zu dem Schluss, dass es besonders in den jüngeren Altersgruppen einen Trend hin zu einer Zunahme der Prävalenz gibt. Sie nehmen an, dass sich ändernde Ernährungsgewohnheiten und Lebensstile ursächlich sein könnten. Nunn et al. (2003) zeigen in einem Vergleich von zwei in Groß-Britannien durchgeführten Untersuchungen, dass die Prävalenz für Erosionen bei $3 \frac{1}{2}$ - bis 4 1/2-Jährigen in einem Zeitraum von 4 Jahren angestiegen ist. Der Anstieg der Prävalenz in dieser Altersgruppe ist mit dem Konsum von Getränken zur Schlafenszeit assoziiert.

Im Vergleich mit neueren internationalen Studien zeigt sich, dass die Prävalenz von $45,4 \%$ etwa dem internationalen Durchschnitt entspricht. Die Spanne der Ergebnisse variiert zwischen 0,6\% (Moimaz et al. 2013) und 98,4\% (Gatou und Mamai-Homata 2012). Mantonanaki et al. (2013) stellten eine Prävalenz von $78,8 \%$ für 5-jährige griechische Vorschulkinder fest. In der vorliegenden Studie waren in dieser Altersgruppe 58,8\% von Erosionen betroffen. Ebenfalls in Grie- 
chenland fanden Gatou und Mamai-Homata (2012) eine Prävalenz für 5- bis 7Jährige von nahezu 100\%. In Brasilien dokumentierten Moimaz et al. (2013) hingegen nur eine Prävalenz von $0,6 \%$ bei 4 - bis 6 -jährigen Vorschulkindern. Ebenfalls in Brasilien fanden Murakami et al. (2011) eine Prävalenz von 51,6\% für 3- bis 4-jährige Kinder. Dabei waren aber 93,9\% der Erosionen auf den Zahnschmelz begrenzt. Tao et al. (2015) stellten in der chinesischen Stadt Shanghai eine Prävalenz von $15,1 \%$ für 3- bis 6-Jährige fest. Davon zeigten lediglich 2,8\% Erosionen mit freigelegter Schmelz-Dentin-Grenze (O'SullivanSchweregrad 3). Nur eins von insgesamt 1837 teilnehmenden Kindern zeigte einen Zahn mit einer bis unter die Schmelz-Dentin-Grenze ausgedehnten Erosion (O'Sullivan-Schweregrad 4). Deutlich verschieden dazu wiesen in der vorliegenden Studie 59,5\% aller von Erosionen betroffenen Kinder mindestens einen Zahn mit dem O'Sullivan-Schweregrad 3 oder höher auf.

Gatou und Mamai-Homata verwendeten zur Klassifikation von Zahnabnutzungen den TWI (Tooth Wear Index) nach Smith und Knight (1984), so dass die Prävalenz von nahezu 100\% nicht nur Erosionen, sondern auch Attritionen und Abrasionen widerspiegelt. Es lässt sich keine Aussage über die Prävalenz von rein erosionsbedingten Zahnhartsubstanzverlusten ableiten. Ein Vergleich mit den Ergebnissen der vorliegenden Untersuchung und anderen Studienergebnissen zur Prävalenz von Erosionen ist deshalb erschwert.

Die Divergenz in den Ergebnissen europäischer Untersuchungen und der brasilianischen Studie von Moimaz et al. (2013) könnte in grundlegenden Länderunterschieden begründet sein. Weitere Studien zu dentalen Erosionen bei 6- bis 12-Jährigen bzw. 12-Jährigen in Brasilien beobachten ebenfalls vergleichsweise geringe Prävalenzen (Peres et al., 2005: 13\%; Mangueira et al. 2009: $19,9 \%)$.

Insgesamt könnten sich für die große Varianz der internationalen Ergebnisse die Anwendung verschiedener Bewertungs-Indizes und eine nicht einheitliche Methodik verantwortlich zeichnen. Ebenfalls könnten landestypische Eigenheiten, wie z. B. Gewohnheiten die Ernährung und die Mundhygiene betreffend, ursächlich sein. Deutlich verschieden sind die Ergebnisse im interkontinentalen Vergleich zwischen Europa, Ostasien und Südamerika. 
5.2.1.2 Prävalenz und Schwere der Erosionen in Abhängigkeit vom Alter

In der vorliegenden Studie wurde eine Zunahme der Prävalenz mit zunehmendem Alter beobachtet. Ebenfalls wiesen ältere Kinder signifikant schwerere Erosionen auf.

Auch Wiegand et al. (2006) und Murakami et al. (2011) beobachteten eine altersabhängige Zunahme der Prävalenz (Tabelle 5.1). Taji et al. (2010) stellten fest, dass die Schwere der Erosionen mit dem Alter signifikant zunimmt. Im Gegensatz dazu zeigten die Untersuchungen von Moimaz et al. (2013) und Tao et al. (2015) keine altersabhängige Zunahme der Prävalenz oder der Schwere von Erosionen.

Der Anstieg der Prävalenz und der Schwere mit zunehmendem Alter spiegelt zum einen den physiologischen Alterungsprozess wider, dem Milchzähne durch ihre anatomische Beschaffenheit im Vergleich zu den bleibenden Zähnen besonders unterliegen. Zum anderen zeigt sich eine pathologische Abnutzung, die bei älteren Kindern durch eine im Zeitverlauf häufigere Exposition gegenüber erosiven Substanzen ausgeprägter sein könnte.

\begin{tabular}{|l|c|c|c|c|}
\hline & 3-Jährige & 4-Jährige & 5-Jährige & 6-Jährige \\
\hline Vorliegende Studie & $14,2 \%$ & $32,9 \%$ & $58,8 \%$ & $71,7 \%$ \\
\hline Wiegand et al. 2006 & $22,5 \%$ & $27,4 \%$ & $30,5 \%$ & $38,1 \%$ \\
\hline Murakami et al. 2011 & $25,5 \%$ & $32,1 \%$ & $/$ & $/$ \\
\hline Mantonanaki et al. 2013 & $/$ & $/$ & $78,8 \%$ & $/$ \\
\hline Moimaz et al. 2013 & $/$ & $0,5 \%$ & $0,3 \%$ & $1.1 \%$ \\
\hline Tao et al. 2015 & $12,0 \%$ & $17,1 \%$ & $16,1 \%$ & $12,6 \%$ \\
\hline
\end{tabular}

Tabelle 5.1: In verschiedenen Studien festgestellte Prävalenzen in den entsprechenden Altersklassen.

5.2.1.3 Prävalenz und Schwere der Erosionen in Abhängigkeit vom Geschlecht In der vorliegenden Studie waren Jungen signifikant häufiger von Erosionen betroffen als Mädchen. Im multilinearen Regressionsmodell zeigte sich, dass in der Gesamtstichprobe Jungen signifikant schwerer von Erosionen betroffen wa- 
ren. Wiegand et al. (2006) beobachteten ebenfalls die Tendenz, dass Jungen häufiger betroffen sind als Mädchen.

Auch internationale Studien zeigen, dass dentale Erosionen und andere nichtkariöse Zahnhartsubstanzverluste bei Jungen häufiger auftreten als bei Mädchen (Tao et al. 2015, Gatou und Mamai-Homata 2012). Gatou und MamaiHomata (2012) stellten zudem fest, dass Jungen schwerer von nichtkariösen Zahnhartsubstanzverlusten betroffen sind als Mädchen. Mantonanaki et al. (2013) und Moimaz et al. (2013) fanden keinen Unterschied in ihren Stichproben.

Studien zur Prävalenz von Erosionen und nichtkariösen Zahnhartsubstanzdefekten bei Jugendlichen beobachten ebenfalls einen Geschlechterunterschied (Al-Dlaigan et al. 2001, van Rijkom et al. 2002, Bardsley et al. 2004). In diesen Studien waren Jungen signifikant häufiger von nichtkariösen Zahnhartsubstanzdefekten betroffen als Mädchen. Als ursächlich werden stärkere Kaukräfte beim männlichen Geschlecht in Kombination mit sauren Nahrungsmitteln und Getränken diskutiert. Jungen könnten außerdem häufiger erosive Getränke konsumieren als Mädchen. Mulic et al. (2012) berichten, dass die Risikofaktoren für Erosionen bei Männern und Frauen differieren. Während bei den Frauen Erbrechen, Reflux und der Konsum von Fruchtsäften für das Auftreten von Erosionen verantwortlich sind, ist es bei den Männern vor allem der häufige Konsum von Softdrinks, der zu Erosionen führt. Eine Untersuchung des Max Rubner-Instituts (2008) bestätigt, dass Männer pro Kopf und Tag mehr als doppelt so viel Limonade konsumieren als Frauen.

5.2.1.4 Prävalenz der Erosionen in Ober- und Unterkiefer und Front- und Seitenzahnbereich

Die vorliegende Studie fand signifikante Unterschiede zwischen der Prävalenz von Ober- und Unterkiefer. Die Milchzähne im Oberkiefer waren mit 25\% fast doppelt so häufig von Erosionen betroffen wie im Unterkiefer mit 13,5\%. Ein ähnliches Bild zeigt sich in der Studie von Wiegand et al. (2006), in der der Oberkiefer mit 11,7\% sogar fast dreimal häufiger als der Unterkiefer mit 3,8\% betroffen ist. Auch Taji et al. (2010) stellten eine unterschiedliche Prävalenz in Ober- und Unterkiefer fest. Während 13\% der Zähne im Unterkiefer betroffen waren, zeigten im Oberkiefer 34\% der Zähne erosive Läsionen. Mantonanaki et 
al. (2013) und Gatou und Mamai-Homata (2012) fanden ebenfalls im Oberkiefer häufiger Erosionen bzw. nichtkariöse Zahnhartsubstanzdefekte als im Unterkiefer. Möglicherweise sind die Zähne des Unterkiefers durch den Speichel der submandibulären und sublingualen Speicheldrüsen geschützter als Ihre Antagonisten im Oberkiefer. Eine Ausnahme stellen die zweiten Milchmolaren des Oberkiefers dar, die über eine anatomisch günstige Lokalisation zur Glandula parotidea verfügen.

In der aktuellen Untersuchung waren die Eckzähne am häufigsten betroffen, gefolgt von den ersten Milchmolaren und den Schneidezähnen Die besonders hohe Prävalenz dentaler Erosionen bzw. nichtkariöser Zahnhartsubstanzdefekte im Bereich der Front- und Eckzähne sowie der ersten Milchmolaren im Oberkiefer findet sich sowohl bei Wiegand et al. (2006), wie auch in weiteren, internationalen Studien (Millward 1994; Taji et al. 2010; Gatou und Mamai-Homata 2012; Mantonanaki et al. 2013). Dass sich in der vorliegenden Untersuchung die Verteilung der Erosionen im Oberkiefer auf den anterioren Bereich konzentrierte, wohingegen im Unterkiefer dentale Erosionen vermehrt auch posterior auftraten, bestätigt die Ergebnisse von Wiegand et al. (2006), Gatou und Mamai-Homata (2012) und Mantonanaki et al. (2013).

Durch die anatomische Lage der Ausführungsgänge der Glandula parotidea im posterioren Bereich der Maxilla scheinen besonders die zweiten Milchmolaren des Oberkiefers geschützter und deshalb weniger von Erosionen betroffen zu sein als die ersten Milchmolaren und die Front- und Eckzähne. Zudem eruptieren die zweiten Milchmolaren später und könnten deshalb weniger von Abnutzung betroffen sein.

\subsubsection{Lokalisation der erosiven Läsionen auf den Zahnflächen}

Hinsichtlich der Lokalisation der Erosionen auf den Zahnflächen zeigte die vorliegende Studie, dass der erosive Zahnhartsubstanzverlust am häufigsten die inzisale bzw. okklusale Zahnfläche betraf (Front- und Eckzähne: 45,5\%; Molaren: 74,2\%). Dies bestätigt die Ergebnisse von Wiegand et al. (2006, Front- und Eckzähne: 51,1\%; Molaren: 75,9\%). International identifizierten Tao et al. (2015), Mantonanaki (2013), Moimaz et al. (2013) und Gatou und MamaiHomata (2012) ebenfalls die inzisale bzw. okklusale Zahnfläche als am häufigsten von Erosionen bzw. anderen nichtkariösen Zahnhartsubstanzverlusten be- 
troffen. Die mit der vorliegenden Studie vergleichbaren Untersuchungen zeichnen somit ein relativ homogenes Bild hinsichtlich der Lokalisation dentaler Erosionen auf den Zahnflächen.

Dass die Frontzähne des Milchgebisses als erstes eruptieren und so im Zeitverlauf öfters mit sauren Getränken Kontakt haben, könnte die Häufigkeit von inzisalen Erosionen an diesen Zähnen begründen. Zudem erscheint es wahrscheinlich, dass der Säurekontakt beim Trinken als erstes die Frontzähne betrifft. Durch den Genuss von erosiven Getränken während der Nahrungsaufnahme könnte die Okklusion mit in den Prozess der Erosion involviert sein. Besonders für die Molaren könnte dies die Häufigkeit der von Erosionen betroffenen okklusalen Flächen erklären. Auch wird diskutiert, dass sich in den teilweise tiefen Fissuren der Molaren erosive Nahrungsmittel länger halten und so die Entstehung von Erosionen auf den okklusalen Flächen begünstigen (Lussi 2006). Da zum Zeitpunkt des Zahndurchbruchs der Milchfrontzähne eine verstärkte Attrition in diesem Bereich beobachtet wird, ist es besonders im späten Stadium des Milchgebisses schwierig, die klinische Abnutzung der inzisalen Flächen ursprünglich ätiologisch zuzuordnen (Mantonanaki et al. 2013). Das klinische Bild könnte bei älteren Kindern auch einen ursprünglich durch Attrition initiierten Zustand zeigen, der im Zeitverlauf erodiert ist.

\subsubsection{Anamnestische Risikofaktoren}

In der vorliegenden Studie war der häufigere Konsum von Limo und Cola signifikant mit schwereren Erosionen assoziiert. Salas et al. (2015) stellten anhand einer Meta-Analyse von 13 ausgewählten Studien fest, dass besonders ein hoher Konsum von Softdrinks mit einem erhöhten Risiko für Erosionen bei Kindern einhergeht. Auch Gatou und Mamai-Homata (2012) fanden heraus, dass der Konsum von Softdrinks mit einer höheren Prävalenz und schwereren Zahnabnutzungen korreliert. Dieser Zusammenhang wird von zahlreichen weiteren internationalen Studien bestätigt (Järvinen et al. 1991, Millward et al.1994, Correr et al. 2009, Huew et al. 2012). Andere Getränke, die in vergleichbaren Studien als signifikante Risikofaktoren für Erosionen identifiziert werden, sind Fruchtsäfte (Luo et al. 2005, Nayak et al. 2012), Buttermilch (Nayak et al. 2012) und Tee und Kaffee (Tao et al. 2015). Das erosive Potential eines Getränks wird von verschiedenen chemischen Faktoren bestimmt. Neben 
dem pH-Wert beeinflussen die Pufferkapazität, die titrierbare Gesamtsäure, die Säure-Art, der pKs-Wert, die chelatisierenden Eigenschaften, die Konzentration anorganischer Elemente (Kalzium, Phosphat und Fluorid), die Temperatur und die Adhäsionsfähigkeit auf der Zahnoberfläche die Erosivität eines Getränks (Barbour und Lussi 2014).

Weitere Ernährungsgewohnheiten, die in internationalen Studien als Risikofaktoren für Erosionen bei Kindern bis 7 Jahre herausgearbeitet werden, sind der tägliche Konsum von Fruchtmus (Harding et al. 2003) und Tamarindenfrucht und Stachelbeeren (Nayak et al. 2012). Ebenfalls werden die Dauer, während der Getränke vor dem Schlucken im Mund gehalten werden (Al-Majed 2002), der Gebrauch eines Fläschchens (Huang et al. 2015) und der Konsum von Essig (Tao et al. 2015) als erosionsfördernd beschrieben. Milch wiederum wirkt sich tendenziell protektiv aus (Wiegand et al. 2006). Nahrungsmittel, die wie Milch und Käse einen hohen Gehalt an Kalzium und Phosphat aufweisen, reduzieren das erosive Potential einer Säure (Linnett und Seow 2001). Diese Ergebnisse konnten von der vorliegenden Studie nicht bestätigt werden.

Kinder, die nach dem abendlichen Zähneputzen Speisen oder Getränke zu sich nahmen, waren in der vorliegenden Untersuchung häufiger von Erosionen betroffen. Dies bestätigt die Ergebnisse von Al-Majed et al. (2002), die einen positiven Zusammenhang zwischen der Zufuhr von Nahrungsmitteln und Softdrinks zur Schlafenszeit und der Häufigkeit von Erosionen bei den oberen Schneidezähnen der ersten und permanenten Dentition fanden. Auch Luo et al. (2005) stellten fest, dass der Konsum von Fruchtsaft zur Schlafenszeit via Fläschchen mit dem Vorkommen von dentalen Erosionen positiv korreliert. In diesen Ergebnissen spiegeln sich zum einen die Erosivität von Softdrinks und Fruchtsäften und zum anderen die Folgen der Einwirkzeit dieser Getränke auf die Zähne wider. Al-Majed et al. (2002) beobachteten, dass die Anzahl bleibender Frontzähne mit palatinalen Erosionen sowohl signifikant mit dem nächtlichen Konsum von Getränken als auch mit der Dauer, mit der diese im Mund gehalten werden, assoziiert sind. Da die meisten Kinder vor dem Schlafengehen ihre Zähne putzen oder geputzt bekommen, steht in diesem Zusammenhang zur Diskussion, dass eine frisch geputzte und von Plaque befreite Zahnoberfläche anfälliger für die Entstehung von Erosionen sein könnte. Ebenfalls wird diskutiert, dass die 
persistierende zervikale Schmelzleiste eines erodierten Zahnes in einer schützenden Plaque-Schicht begründet sein könnte (Lussi et al. 2006). Studien zeigen, dass die Pellikel in Abhängigkeit von ihrer Dicke und Reifungszeit die Zahnhartsubstanzen bis zu einem gewissen Grad vor erosiven Säureangriffen schützen kann (Amaechi et al. 1999b, Hannig et al. 2003, Wiegand et al. 2008). Die Pellikel wird durch das Putzen der Zähne von den Zahnoberflächen entfernt, beginnt aber unmittelbar danach, sich wieder flächendeckend aus Speichelproteinen zu bilden.

In einigen Studien wird der Einfluss des Mundhygieneverhaltens auf die Entstehung von Erosionen untersucht. Mantonanaki et al. (2013) zeigten, dass eine gute Mundhygiene das Risiko für Erosionen erhöht. Sie bestätigten damit die Ergebnisse von Nayak et al. (2012), die feststellten, dass das mindestens zweimalige oder häufigere Zähneputzen pro Tag mit Erosionen assoziiert ist. Dieser Effekt wird von den Autoren mit dem Wegbürsten der Pellikel erklärt. Wiegand et al. (2006) berichten hingegen, dass Kinder bis 7 Jahre ein höheres Risiko für Erosionen haben, wenn sie selbst ihre Zähne putzen, ohne dass die Eltern nachputzen. Es ist davon auszugehen, dass Kinder bis 7 Jahre ohne Hilfe der Eltern nur eine insuffiziente Entfernung der Plaque vornehmen können. Aus diesem Grund wird empfohlen, Kindern bis 10 Jahren die Zähne nachzuputzen (Sandström et al. 2011). Al-Malik et al. (2002) fanden einen Zusammenhang zwischen dem Vorliegen von Karies und dem Risiko, gleichzeitig auch Erosionen zu entwickeln.

Einige Studien erkennen sozioökonomische Faktoren als ursächlich für die Entstehung von Erosionen. Diese Ergebnisse sind allerdings widersprüchlich. Harding et al. (2003) zeigten, dass ein niedriges Familieneinkommen mit Erosionen assoziiert ist, während Mantonanaki et al. (2013) das Gegenteil beobachteten. Weiterhin wurden ein hoher sozioökonomischer Status und ein niedriger Bildungsgrad der Mutter als Risikofaktoren herausgearbeitet (Millward et al. 1994, Mantonanaki et al. 2013). Gatou und Mamai-Homata (2012) fanden heraus, dass die Immigranten in ihrer Stichprobe eine höhere Prävalenz für Zahnabnutzungen hatten als die griechische Vergleichsgruppe. Auch Tao et al. (2015) stellten fest, dass der Geburtsort einen signifikanten Einfluss auf das Vorkommen von Erosionen hat. 
Des Weiteren wird verifiziert, dass intrinsische Faktoren signifikante Risikofaktoren für Erosionen darstellen. Es werden gastroösophagealer Reflux (Murakami et al. 2011) und die zu den Essstörungen zählende Regurgitation (Tao et al. 2015) als Risikofaktoren identifiziert. Diese Ergebnisse konnten aufgrund zu weniger Beobachtungen von der vorliegenden Studie nicht bestätigt werden. Jaeggi und Lussi (2004) und Moimaz et al. (2013) stellten in ihren Untersuchungen keine signifikanten Risikofaktoren fest.

\subsubsection{Rolle der Kindergärten}

Die Auswertung der Kindergarten-Fragebögen konnte keine kindergartenspezifischen Risikofaktoren identifizieren. Möglicherweise resultiert dies aus den geringen Unterschieden in den Angaben der Kindergärten hinsichtlich der angebotenen Ernährung und Mundhygiene.

\subsection{Fazit und Ausblick}

Im Rahmen dieser Untersuchung war es möglich zu zeigen, dass dentale Erosionen bereits im Milchgebiss häufig auftreten. Fast jedes zweite Göttinger Kindergartenkind war von Erosionen betroffen. Bei etwa jedem 20. Kind wurden Erosionen mit einem Schweregrad diagnostiziert, die nach den Empfehlungen von Bartlett et al. (2008) eine restaurative Behandlung erforderlich machen. Innerhalb der vorliegenden Studie konnte außerdem gezeigt werden, dass sich der Konsum kohlensäurehaltiger Softdrinks auf die Schwere von Erosionen auswirkt. Besonders Erosionen mit einem hohen Schweregrad waren mit dem Konsum von Limo und Cola assoziiert. Die Ergebnisse dieser Studie unterstreichen somit die Notwendigkeit weiterer Forschung, speziell im Hinblick auf geeignete zahnmedizinische Präventionsmaßnahmen bei Kleinkindern und Kindern im Milchgebiss. Eltern und Kinder sollten schon früh über die Entstehung von dentalen Erosionen im Milchgebiss und die Erosivität von kohlensäurehaltigen Softdrinks aufgeklärt werden. Kinder, die von Erosionen betroffen sind, sollten den Empfehlungen von Bartlett et al. (2008) entsprechend zahnärztlich betreut bzw. therapiert und in ein Recall-Programm aufgenommen werden. 


\section{Zusammenfassung}

Ziel der Studie: Die Ziele der vorliegenden Studie waren, die Prävalenz, die Schwere und die Lokalisation von dentalen Erosionen bei Göttinger Kindergartenkindern zwischen 3 und 7 Jahren zu ermitteln. Außerdem wurde eine Risikofaktoranalyse durchgeführt.

Patienten und Methode: An der Studie nahmen 777 Kinder zwischen 3 und 7 Jahren teil. Zur Klassifikation der Erosionen wurden der O'Sullivan-Index (O'Sullivan 2000) und der BEWE-Index (Basic Erosive Wear Examination, Bartlett et al. 2008) herangezogen. Mit einem Anamnesebogen wurden Allgemeinerkrankungen, Medikation, Mundhygiene- und Ernährungsgewohnheiten erfragt. Des Weiteren wurden mit einem Fragebogen Daten zu Mundhygiene- und Ernährungsgewohnheiten in den Kindergärten erhoben.

Ergebnisse: 45,4\% der 777 untersuchten Kinder wiesen mindestens einen Milchzahn mit Erosionen auf. Die Zähne der untersuchten bleibenden Dentition waren nicht betroffen. Die Prävalenz der Erosionen nahm mit zunehmendem Alter signifikant zu: 3-Jährige: 14,2\%; 4-Jährige: 32,9\%; 5-Jährige: 58,8\%; 6-7Jährige: $71,4 \%$. Ebenfalls nahm die Schwere der Erosionen mit dem Alter zu. Jungen waren signifikant häufiger und schwerer von Erosionen betroffen als Mädchen. Die Verteilung der 777 Kinder entsprechend den BEWERisikogruppen zeigte, dass $66,4 \%$ kein, $28,1 \%$ ein niedriges, $5,0 \%$ ein mittleres und $0,5 \%$ ein hohes Risiko für Erosionen hatten. 59,5\% der betroffenen Kinder zeigten Erosionen mit Beteiligung des Dentins. Die Oberkieferzähne waren häufiger $(43,4 \%)$ und schwerer von Erosionen betroffen als die Unterkieferzähne (28,3\%). Im Oberkiefer waren die Eckzähne mit 31,5 - 31,7\% und die ersten Milchmolaren mit 30,0 - 30,2\%, im Unterkiefer die Eckzähne mit 17\% und die ersten Milchmolaren mit 16,5 - 17,7\% am häufigsten betroffen. Der häufigere Konsum von Limo und Cola war mit schwereren Erosionen assoziiert. Kinder, die nach dem abendlichen Zähneputzen Speisen oder Getränke zu sich nahmen, waren häufiger von Erosionen betroffen. Durch Auswertung der Kindergarten-Fragebögen konnte kein weiterer Risikofaktor identifiziert werden.

Schlussfolgerung: Knapp die Hälfte der untersuchten Kinder war von erosiven Zahnhartsubstanzverlusten betroffen, wobei ein kleiner Teil der Gesamtstich- 
probe ein mittleres bis hohes Risiko für Erosionen zeigte. Der Konsum von Limo und Cola sowie die Zufuhr von Speisen und Getränken nach dem abendlichen Zähneputzen konnten als Risikofaktoren für Erosionen identifiziert werden. 
$7 \quad$ Anhang

\subsection{Anamnesebogen und Kindergarten-Fragebogen}

\section{UNIVERSITÄTSMEDIZIN $* \mathbf{M} \mathbf{M G}$
GÖTTINGEN}

Zentrum Zahn-, Mund- und Kieferheilkunde Poliklinik für Präventive Zanhmedizin Parodontologie und Kariologie

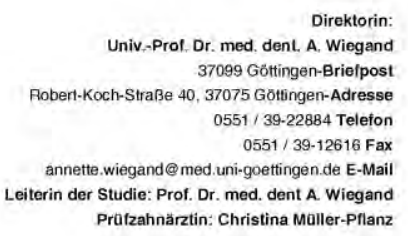

Direktorin:

Univ-Prot Dr. med. dent. A. Wiegand 37099 Göttingen-Brietpost Robert-Koch-Straße 40, 37075 Göttingen-Adresse 0551/39-22884 Telefon $0551 / 39-12616 \mathrm{Fax}$

annette.wiegand@med. uni-goettingen.de E-Mail Leiterin der Studie: Prof. Dr. med. dent A. Wiegand Prutzahnärztin: Christina Maller-Pflanz

\section{Fragebogen zur Studie:}

Prävalenz von und Risikofaktoren für dentale Erosionen bei Göttinger Kindergarten-Kindern

Code:

Geburtsmonat/-jahr:

Geschlecht: weiblich imännlich

Allgemeinerkrankungen und Medikation:

- Diabetes (_Zucker")

- Lungenerkrankungen/Asthma

Wenn ja, nimmt Ihr Kind zur Therapie regelmäßig ein Inhalationsspray?

Reflux (,Sodbrennen“)

Wenn ja, wie hăufig? mal pro Monat

- Erkrankungen, die mit häufigem Erbrechen einhergehen

Wenn ja, wie häufig wird erbrochen?

mal pro Monat

- Andere Allgemeinerkrankungen

Wenn ja, welche?

- Muss Ihr Kind regelmäßig Medikamente einnehmen?

Wenn ja, welche?

Und seit wann?

- Nimmt oder nahm Ihr Kind regelmäßig Fluoridtabletten ein?

Wenn ja, welche?

Und seit wann?

vorn

bis

Ernährungsgewohnheiten:

- Wurde Ihr Kind als Baby gestillt? Wenn ja, wie lange? vom..................... bis zum

- Hat Ihr Kind das Fläschchen bekommen oder bekommt es noch? Wenn ja, mit weichem Inhalt? 


\section{UNIVERSITÄTSMEDIZIN :
GÖTTINGEN : $\mathbf{M G}$}

- Bekam oder bekommt lhr Kind ein Fläschchen zum Einschlafen?

๖ja

anein

Wenn ja, mit welchem Inhalt?

- Wie häufig nimmt ihr Kind folgende Getränke und Nahrungsmittel zu sich:

\begin{tabular}{|l|c|c|c|c|c|}
\hline Fruchtsäfte pur & $\square$ nie/selten & $\square$ wöchentlich & $\square$ täglich & $\square 2-3$ mal täglich & $\square$ häufiger \\
\hline Fruchtsäfte verdünnt & $\square$ nie/selten & $\square$ wöchentlich & $\square$ täglich & $\square 2-3$ mal täglich & $\square$ häufiger \\
\hline Zitronentee & $\square$ nie/selten & $\square$ wöchentlich & $\square$ täglich & $\square 2-3$ mal täglich & $\square$ häufiger \\
\hline Limonade / Cola & $\square$ nie/selten & $\square$ wöchentlich & $\square$ täglich & $\square 2-3$ mal täglich & $\square$ häufiger \\
\hline Kohlensäurehaltiges Mineralwasser & $\square$ nie/selten & $\square$ wöchentlich & $\square$ täglich & $\square 2-3$ mal täglich & $\square$ häufiger \\
\hline Stilles Mineralwasser & $\square$ nie/selten & $\square$ wöchentlich & $\square$ täglich & $\square 2-3$ mal täglich & $\square$ häufiger \\
\hline Milch & $\square$ nie/selten & $\square$ wöchentlich & $\square$ täglich & $\square$ 2-3 mal täglich & $\square$ häufiger \\
\hline Zitrusfrüchte & $\square$ nie/selten & $\square$ wöchentlich & $\square$ täglich & 2-3 mal täglich & $\square$ häufiger \\
\hline Alle Arten von Süßigkeiten & $\square$ nie/selten & $\square$ wöchentlich & $\square$ täglich & $\square 2-3$ mal täglich & $\square$ häufiger \\
\hline Zitronensprudel & $\square$ nie/selten & $\square$ wöchentlich & $\square$ täglich & $\square 2-3$ mal täglich & $\square$ häufiger \\
\hline
\end{tabular}

- Wie nimmt lhr Kind oben genannte Getränke zu sich?

\begin{tabular}{|l|c|c|c|c|c|}
\hline Aus einem Becher & $\square$ nie & $\square$ selten & $\square$ manchmal & $\square$ oft & $\square$ immer \\
\hline Mit Strohhalm & $\square$ nie & $\square$ selten & $\square$ manchmal & $\square$ oft & $\square$ immer \\
\hline Mit Trinklernbecher/Schnabeltasse & $\square$ nie & $\square$ selten & $\square$ manchmal & $\square$ oft & $\square$ immer \\
\hline Aus dem Fläschchen & $\square$ nie & $\square$ selten & $\square$ manchmal & $\square$ oft & immer \\
\hline
\end{tabular}

Mundhygienemaßnahmen:

- Wer putzt die Zähne Ihres Kindes?
$\square$ das Kind selbst
die Eltern
口uerst Kind selbst, dann putzen Eltern nach

- Wie oft putzt sich Ihr Kind die Zähne / werden Ihm die Zähne geputzt?
1 mal täglich
(1) 2 mal täglich
c. 3 mal täglich
20.....mal wöchentlich

- Wie lange putzt Ihr Kind / putzen Sie Ihrem Kind die Zähne?
1 Minute
2 Minuten
๘ 3 Minuten

- Welche Art Zahnbürste benutzt Ihr Kind / benutzen Sie für Ihr Kind?
$\square$ eine Handzahnbürste
q eine elektrische Zahnbürste
$\square$ beides

- Welche Zahnpasta benutzt Ihr Kind / benutzen Sie für Ihr Kind?

$\square$ fluoridierte Kinder-Zahnpasta

nicht-fluoridierte Kinder-Zahnpasta

口fluoridierte Erwachsenen-Zahnpasta

anicht-fluoridierte Erwachsenen-Zahnpastå

- Nimmt Ihr Kind abends nach dem Zähneputzen noch Speisen und/oder Getränke zu sich?
घja
1 nein
Wenn ja, wie oft?
väglich
Q wöchentlich
monatlich

Und welche?

Universitätsmedizin Göttingenen, Georg-AugusstUniversitżt 5tittung Oittentlichen Rechits Vorstand Prol. Ds. Heyo K. Kroemer (Forschung \& Letre- Sprecher des Vorstands) 2

Dr. Martio Siess (Krankenversorgung) Dr. Sedastian Freytag (Wirtschaftslüriung \& Administration) Sparkasse Göttingen (26050001) Rto.448

Abbildung 7.2: Anamnesebogen, von den Erziehungsberechtigten auszufüllen: Seite 2. 


\title{
UNIVERSITÄTSMEDIZIN $\approx$
GÖTTINGEN $=\bigcup \mathbf{M G}$
}

\author{
Zentrum Zahn-, Mund- und Kieferheilkunde \\ Poliklinik für Präventive Zahnmedizin, \\ Parodontologie und Kariologie \\ Direktorin: \\ Univ.-Prof. Dr. med. dent. A. Wiegand \\ 37099 Gồttingen Briefpost \\ Robert-Koch-Straße 40, 37075 Göttingen Adresse \\ 0551 / 39-22884 Telefon \\ $0551 / 39-12616 \mathrm{Fax}$ \\ annette.wiegand@med.uni-goettingen,de E-Mail
}

Leiterin der Studie: Prof. Dr. med. dent. A. Wiegand Prolzahnärztin: Christina Maller-Pflanz

\section{Fragebogen zur Studie:}

Prävalenz von und Risikofaktoren für dentale Erosionen bei Göttinger Kindergarten-Kindern

Name der Einrichtung:

\section{Ernährungsgewohnheiten:}

- Welche Getränke und Speisen werden für alle Kinder in Ihrer Einrichtung angeboten (inklusive Kindergeburtstagen und besonderen Anlässen wie z.B. Weihnachtsfeiern etc.)?

\begin{tabular}{|l|c|c|c|c|c|}
\hline Fruchtsäfte pur & $\square$ nie/selten & $\square$ wöchentlich & $\square$ täglich & $\square 2-3$ mal täglich & $\square$ häufiger \\
\hline Fruchtsäfte verdünnt & $\square$ nie/selten & $\square$ wöchentlich & $\square$ täglich & $\square 2-3$ mal täglich & $\square$ häufiger \\
\hline Zitronentee & $\square$ nie/selten & $\square$ wöchentlich & $\square$ täglich & $\square 2-3$ mal täglich & $\square$ häufiger \\
\hline Limonade/ Cola & $\square$ nie/selten & $\square$ wöchentlich & $\square$ täglich & $\square 2-3$ mal täglich & $\square$ häufiger \\
\hline $\begin{array}{l}\text { Kohlensäurehaltiges } \\
\text { Mineralwasser }\end{array}$ & $\square$ nie/selten & $\square$ wöchentlich & $\square$ täglich & $\square 2-3$ mal täglich & $\square$ häufiger \\
\hline Stilles Mineralwasser & $\square$ nie/selten & $\square$ wöchentlich & $\square$ täglich & $\square 2-3$ mal täglich & $\square$ häufiger \\
\hline Milch & $\square$ nie/selten & $\square$ wöchentlich & $\square$ täglich & $\square 2-3$ mal täglich & $\square$ häufiger \\
\hline Zitrusfrüchte & $\square$ nie/selten & $\square$ wöchentlich & $\square$ täglich & $\square 2-3$ mal täglich & $\square$ häufiger \\
\hline Alle Arten von Süßigkeiten & $\square$ nie/selten & $\square$ wöchentlich & $\square$ täglich & $\square 2-3$ mal täglich & $\square$ häufiger \\
\hline Zitronensprudel & $\square$ nie/selten & $\square$ wöchentlich & $\square$ täglich & $\square 2-3$ mal täglich & $\square$ häufiger \\
\hline
\end{tabular}

Abbildung 7.3: Fragebogen, von den Kindergärten auszufüllen: Seite 1. 


\section{UNIVERSITÄTSMEDIZIN $\equiv \mathbf{M} \mathbf{G}$
GÖTTINGEN $=0$}

- Wie werden oben genannte Getränke konsumiert?

\begin{tabular}{|l|c|c|c|c|c|}
\hline Aus einem Becher & $\square$ nie & $\square$ selten & $\square$ manchmal & $\square$ oft & $\square$ immer \\
\hline Mit Strohhalm & $\square$ nie & $\square$ selten & $\square$ manchmal & $\square$ oft & $\square$ immer \\
\hline Mit Trinklembecher/Schnabeltasse & $\square$ nie & $\square$ selten & $\square$ manchmal & $\square$ oft & $\square$ immer \\
\hline Aus dem Fläschchen & $\square$ nie & $\square$ selten & $\square$ manchmal & $\square$ oft & $\square$ immer \\
\hline
\end{tabular}

\section{Mundhygienemaßnahmen:}

- Findet in Ihrer Einrichtung tägliches Zähneputzen statt?

$\begin{array}{lll} & \square \text { ja } & \square \text { nein } \\ \text { Wenn ja, wie oft? } \square 1 \mathrm{mal} & \square 2 \mathrm{mal} \quad \square \text { häufiger }\end{array}$

-Wer putzt die Zähne der Kinder? $\square$ die Kinder selbst

$\square$ die Erzieher/innen

口 erst die Kinder, dann wird von den Erzieher/innen

nachgeputzt

- Wie lange putzen die Kinder die Zähne bzw. werden Ihnen die Zähne geputzt?

$$
\begin{aligned}
& \square 1 \text { Minute } \\
& \square 2 \text { Minuten } \\
& \square 3 \text { Minuten }
\end{aligned}
$$

- Welche Zahnpasta wird von den Kindern bzw. für die Kinder benutzt?

$$
\begin{aligned}
& \square \text { fluoridierte Kinder-Zahnpasta } \\
& \square \text { nicht-fluoridierte Kinder-Zahnpasta } \\
& \square \text { fluoridierte Erwachsenen-Zahnpasta } \\
& \square \text { nicht-fluoridierte Erwachsenen-Zahnpasta }
\end{aligned}
$$

- Welche Art Zahnbürste benutzen die Kinder bzw. wird für sie benutzt?

$$
\begin{aligned}
& \square \text { eine Handzahnbürste } \\
& \square \text { eine elektrische Zahnbürste } \\
& \square \text { beides }
\end{aligned}
$$

\section{Abbildung 7.4: Fragebogen, von den Kindergärten auszufüllen: Seite 2.}




\section{Literaturverzeichnis}

Al-Dlaigan YH, Shaw L, Smith AJ (2001): Dental erosion in a group of British 14-year-old school children. Part 1. Prevalence and influence of differing socioeconomic backgrounds. Br Dent J 190, 145-149

Al-Dlaigan YH, Shaw L, Smith AJ (2002): Is there a relationship between asthma and dental erosion? A case control study. Int J Paediatr Dent 12, 189-200

Al-Majed I, Maguire A, Murray JJ (2002): Prevalence and risk factors for dental erosion in 5-6-year-old and 12-14-year-old boys in Saudi Arabia. Community Dent Oral Epidemiol $\underline{30}, 38-46$

Al-Malik MI, Holt RD, Bedi R, Speight PM (2002): Erosion, caries and rampant caries in preschool children in Jeddah, Saudi Arabia. Community Dent Oral Epidemiol $\underline{30}, 16-23$

Alvarez Loureiro L, Fabruccini Fager A, Alves LS, Alvarez Vaz R, Maltz M (2015): Erosive tooth wear among 12-year-old schoolchildren: a populationbased cross-sectional study in Montevideo, Uruguay. Caries Res $\underline{49}$, 216-225

Alves LS, Brusius CD, Damé-Teixeira N, Maltz M, Susin C (2015): Dental erosion among 12-year-old schoolchildren: a population-based cross-sectional study in South Brazil. Int Dent J $\underline{65}, 322-330$

Amaechi BT, Higham SM, Edgar WM (1999a): Factors influencing the development of dental erosion in vitro: enamel type, temperature and exposure time. J Oral Rehabil 26, 624-630

Amaechi BT, Higham SM, Edgar WM, Milosevic A (1999b): Thickness of aquired salivary pellicle as a determinant of the sites of dental erosion. J Dent Res $\underline{78}, 1821-1828$

Amin WM, Al-Omoush SA, Hattab FN (2001): Oral health status of workers exposed to acid fumes in phosphate and battery industries in Jordan. Int Dent $J$ 51, 169-174

Arnadottir AB, Saemundsson SR, Holbrook WP (2003): Dental erosion in icelandic teenagers in relation to dietary and lifestyle factors. Acta Odontol Scand $\underline{61}, 25-28$ 
Attin T, Deifuss H, Hellwig E (1999): Influence of acidic fluoride gel on abrasion resistance of eroded enamel. Caries Res $\underline{33}, 135-139$

Attin T, Buchalla W, Gollner M, Hellwig E (2000): Use of variable remineralization periods to improve the abrasion resistance of previously eroded enamel. Caries Res $\underline{34}$, 48-52

Attin T, Wegehaupt F, Gries D, Wiegand A (2007): The potential of deciduous and permanent bovine enamel as substitute for deciduous and permanent human enamel: erosion-abrasion experiments. J Dent $\underline{35}, 773-777$

Barbour ME, Lussi A: Erosion in relation to nutrition and the environment. In: Lussi A, Ganss C (eds.): Erosive tooth wear. Monograph Oral Sci. Karger, Basel 2014, 25, 143-154

Bardsley PF (2008): The evolution of tooth wear indices. Clin Oral Investig $\underline{12}$, $15-19$

Bardsley PF, Taylor S, Milosevic A (2004): Epidemiological studies of tooth wear and dental erosion in 14-year-old children in North West England. Part 1: the relationship with water fluoridation and social deprivation. Br Dent J $\underline{197}$, 413-416

Bartlett D, Ganss C, Lussi A (2008): Basic Erosive Wear Examination (BEWE): a new scoring system for scientific and clinical needs. Clin Oral Investig $\underline{12}, 65-$ 68

Bartlett DW, Coward PY (2001): Comparison of the erosive potential of gastric juice and a carbonated drink in vitro. J Oral Rehab $\underline{28}, 1045-1047$

Bartlett DW, Evans DF, Anggiansah A, Smith BG (1996): A study of the association between gastro-oesophageal reflux and palatal dental erosion. $\mathrm{Br}$ Dent $\mathrm{J}$ 181, 125-131

Bartlett DW, Coward PY, Nikkah C, Wilson RF (1998): The prevalence of tooth wear in a cluster sample of adolescent schoolchildren and its relationship with potential explanatory factors. Br Dent J $\underline{184}, 125-129$ 
Bartlett DW, Fares J, Shirodaria S, Chiu K, Ahmad N, Sheriff M (2011): The association of tooth wear, diet and dietary habits in adults aged $18-30$ years old. $\mathrm{J}$ Dent $\underline{39}, 811-816$

Benages A, Muñoz JV, Sanchiz V, Mora F, Minguez M (2006): Dental erosion as extraoesophageal manifestation of gastro-oesophageal reflux. Gut $\underline{55}, 1050-$ 1551

Bere E, Glomnes ES, Te Velde SJ, Klepp KI (2008): Determinants of adolescents' soft drink consumption. Public Health Nutr $\underline{11}$, 49-56

Blacker SM, Chadwick RG (2013): An in vitro investigation of the erosive potential of smoothies. Br Dent J $\underline{214}$, E9

Breschi L, Gobbi P, Mazzotti G, Falconi M, Ellis TH, Stangel I (2002): High resolution SEM evaluation of dentin etched with maleic and citric acid. Dent Mater 18, 26-35

Brosowsky A (1966): Die Wirkung von organischen und Mineralsäuren auf die Zahnhartsubstanz bei verschiedenen pH-Werten. Dtsch Zahnärztl Z 21, 11391147

Buczkowska-Radlińska J, Łagocka R, Kaczmarek W, Górski M, Nowicka A (2013): Prevalence of dental erosion in adolescent competetive swimmers exposed to gas-chlorinated swimming pool water. Clin Oral Investig $\underline{17}$, 579-583

Caglar E, Kargul B, Tanboga I, Lussi A (2005): Dental erosion among children in an Istanbul public school. J Dent Child $\underline{72}, 5-9$

Caglar E, Sandalli N, Panagiotou N, Tonguc K, Kuscu OO (2011): Prevalence of dental erosion in greek minority school children in Istanbul. Eur Arch Paediatr Dent $\underline{12}, 267-271$

Centerwall BS, Armstrong CW, Funkhouser LS, Elzay RP (1986): Erosion of dental enamel among competetive swimmers at a gas-chlorinated swimming pool. Am J Epidemiol 123, 641-647

Chu CH, Ng A, Chau AM, Lo EC (2015): Dental erosion and caries status of chinese university students. Oral Health Prev Dent $\underline{13}$, 237-244 
Correa MC, Lerco MM, Cunha ML, Henry MA (2012): Salivary parameters and teeth erosions in patients with gastroesophageal reflux desease. Arq Gastroenterol $\underline{49}$, 214-218.

Correr GM, Alonso RC, Consani S, Puppin-Rontani RM, Ferracane JL (2007): In vitro wear of primary and permanent enamel. Simultaneous erosion and abrasion. Am J Dent 20, 394-399

Correr GM, Alonso RC, Correa MA, Campos EA, Baratto-Filho F, PuppinRontani RM (2009): Influence of diet and saliva characteristics on the prevalence of dental erosion among 12-year-old schoolchildren. J Dent Child $\underline{76}, 181$ 187

Di Fede O, Di LC, Occhipinti G, Vigneri S, Lo RL, Fedele S, Lo Muzio L, Campis $G$ (2008): Oral manifestations in patients with gastroesophageal reflux desease: a single-center case-control study. J Oral Pathol Med $\underline{37}, 336-340$

Donachie MA, Walls AW (1995): Assessment of tooth wear in an ageing population. J Dent $\underline{23}, 157-164$

Dreizen S, Brown LR, Daly TE, Drane JB (1977): Prevention of xerostomiarelated dental caries in irradiated cancer patients. J Dent Res $\underline{56}, 99-104$

Dugmore CR, Rock WP (2003a): The progression of tooth erosion in a cohort of adolescents of mixed ethnicity. Int J Paediatr Dent $\underline{13}$, 295-303

Dugmore CR, Rock WP (2003b): Asthma and tooth erosion. Is there an association? Int J Paediatr Dent $\underline{13}$, 417-424

Dukic W, Dobrijevic TT, Katunaric M, Milardovic S, Segovic S (2010): Erosive lesions in patients with alcoholism. J Am Dent Assoc 141, 1452-1458

Eccles JD (1979): Dental erosion of non-industrial origin. A clinical survey and classification, J Prosthet Dent $\underline{42}, 649-653$

Eccles JD, Jenkins WG (1974): Dental erosion and diet. J Dent 2 2, 153-159

El-Aidi H, Bronkhorst EM, Huysmans MC, Truin GJ (2010): Dynamics of tooth erosion in adolescents: a 3-year longitudinal study. J Dent $\underline{38}, 131-137$ 
Engelen L, de Wijk RA, Prinz JF, van der Bilt A, Bosman F (2003): The relation between saliva flow after different stimulations and the perception of flavor and texture attributes in custard desserts. Physiol Behav $\underline{78}, 165-169$

Ganss C, Lussi A: Diagnosis of Erosive Tooth Wear. In: Lussi A, Ganss C (eds.): Erosive tooth wear. Monograph Oral Sci. Karger, Basel 2014, 25, 22-31

Ganss C, Schlechtriemen M, Klimek J (1999): Dental erosions in subjects living on a raw food diet. Caries Res $\underline{33}, 74-80$

Ganss C, Klimek J, Giese K (2001): Dental erosion in children and adolescents - a cross-sectional and longitudinal investigating using study models. Community Dent Oral Epidemiol $\underline{29}$, 264-271

Ganss C, Klimek J, Starck C (2004): Quantitative analysis of the impact of the organic matrix on the fluoride effect on erosion progression in human dentine using longitudinal microradiography. Arch Oral Biol $\underline{49}$, 931- 935

Ganss C, Hardt M, Blazek D, Klimek J, Schlueter N (2009): Effects of tooth brushing force on the mineral content and demineralised organic matrix of eroded dentine. Eur J Oral Sci 117, 255-260

Ganss C, Hardt M, Lussi A, Cocks AK, Klimek J, Schlueter N (2010): Mechanism of action of tin-containing fluoride solutions as anti-erosive agents in dentine - an in-vitro tin uptake, tissue loss and SEM study. Eur J Oral Sci $\underline{118}, 376-$ 384

Gatou T, Mamai-Homata E (2012): Tooth wear in deciduous dentition of 5-7year-old children: risk factors. Clin Oral Investig $\underline{16}$, 923-933

González-Aragón ÁE, Borges-Yáñez SA, Lussi A, Irigoyen-Camacho ME, Angeles Medina $F$ (2016): Prevalence of erosive tooth wear and associated factors in a group of mexican adolescents. J Am Dent Assoc 147, 92-97

Graehn G (1991): Acid erosions of tooth surface. 1. Influence of saliva, medicaments, fruit acids and mechanical stress. Dtsch Stomatol $\underline{41}, 494-499$

Graf F (1953): Über die Entkalkung des Zahnschmelzes durch Fruchtsäuren und Tafelgetränke. Schweiz Monatsschr Zahnheilkd $\underline{63}$, 3-32 
Gregg T, Mace S, West NX, Addy M (2004): A study in vitro of the abrasive effect of the tongue on enamel and dentine softened by acid erosion. Caries Res $\underline{38}, 557-560$

Greve W, Wentura D: Wissenschaftliche Beobachtung: Eine Einführung. PVU/Beltz, Weinheim 1997

Grine FE (2005): Enamel thickness of deciduous and permanent molars in modern Homo sapiens. Am J Phys Anthropol 126, 14-31

Hannig M, Joiner A (2006): The structure, function and properties of the acquired pellicle; in Druckworth RM (ed): The teeth and their environment. Monogr Oral Sci $\underline{19}, 29-64$

Hannig M, Hess NJ, Hoth-Hannig W, de Vrese M (2003): Influence of salivary pellicle formation time on enamel demineralization - an in situ pilot study. Clin Oral Invest $\underline{7}, 158-161$

Hara AT, Ando M, Cury JA, Serra MC, Gonzalez-Cabezas C, Zero D (2005): Influence of the organic matrix on root dentine erosion by citric acid. Caries Res $\underline{39}, 134-138$

Hara AT, Karlinsey RL, Zero DT (2008): Dentine remineralization by stimulated saliva formulations with different $\mathrm{Ca}$ and $\mathrm{P}$ contents, Caries Res $\underline{42}, 51-56$

Harding MA, Whelton H, O'Mullane DM, Cronin M (2003): Dental erosion in 5year-old irish school children and associated risk factors: a pilot study. Community Dent Health $\underline{20}, 165-170$

Hasselkvist A, Johansson A, Johansson AK (2010): Dental erosion and soft drink consumption in swedish children and adolescents and the development of a simplified erosion partial recording system. Swed Dent J $\underline{34}$, 187-195

Hotz PR (1987): Erosion des Zahnschmelzes. Schweiz Monatsschr Zahnmed 97, 219-222

Huang LL, Leishman S, Newman B, Seow WK (2015): Association of erosion with timing of detection and selected risk factors in factors in primary dentition: a longitudinal study. Int J Paediatr Dent 25, 165-173 
Huew R, Waterhouse PJ, Moynihan PJ, Maguire A (2012): Dental erosion among 12 year-old libyan schoolchildren. Community Dent Health $\underline{29}$, 279-283

Hunter ML, West NX, Hughes JA, Newcombe RG, Addy M (2000): Erosion of deciduous and permanent dental hard tissue in the oral environment. J Dent $\underline{28}$, 257-263

Imfeld T (1996): Dental erosion. Definition, classification and links. Eur J Oral Sci $\underline{104}, 151-155$

Jaeggi T, Lussi A (2004): Erosionen bei Kindern im frühen Schulalter. Schweiz Monatsschr Zahnmed 114, 876-881

Jaeggi T, Lussi A: Prevalence, incidence and distribution of erosion. In: Lussi A, Ganss C (eds): Erosive tooth wear. Monogr Oral Sci. Karger, Basel 2014, 25, $55-73$

Jaeggi T, Schaffner M, Bürgin W, Lussi, A (1999): Erosionen und keilförmige Defekte bei Rekruten der Schweizer Armee. Schweiz Monatsschr Zahnmed $\underline{109}, 1171-1182$

Järvinen VK, Rytömaa I, Heinonen OP (1991): Risk factors in dental erosion. J Dent Res $\underline{70}$, 942- 947

Järvinen V, Meurman JH, Hyvarinen H, Rytömaa I, Murtomaa H (1988): Dental erosion and upper gastrointestinal disorders. Oral Surg Oral Med Oral Pathol $\underline{65}, 298-303$

Janssens J, Vantrappen G, Ghillebert G (1986): 24-hour recording of esophageal pressure and $\mathrm{pH}$ in patients with noncardiac chest pain. Gastroenterology 90, $1978-1984$

Johansson AK, Sorvari R, Birkhed D, Meurman JH (2001): Dental erosion in deciduous teeth - an in vivo and in vitro study. J Dent $\underline{29}, 333-340$

Jones L, Lekkas D, Hunt D, Mclntyre J, Rafir W (2002): Studies on dental erosion: An in vivo-in vitro model of endogenous dental erosion - its application to testing protection by fluoride gel application. Aust Dent $\mathrm{J} \underline{47}$, 304-308. 
Jordan K, Jahn F, Aapro M (2015): Recent developments in the prevention of chemotherapy-induced nausea and vomiting (CINV): a comprehensive review. Ann Oncol 26, 1081-1090

Kaczmarek U, Czajczynska-Waszkiewicz A, Skladnik-Jankowska J (2012): Prevalence of dental erosion in15-year-old subjects in Lower Silesia province. J Stoma $\underline{65}, 359-369$

Khan F, Young WG, Law V, Priest J, Daley TJ (2001): Cupped lesions of early onset dental erosion in young southeast Queensland adults. Aust Dent $\mathrm{J} \underline{46}$, 100-107

Kim H-D, Douglass CW (2003): Associations between occupational health behaviors an occupational dental erosion. J Public Health Dent $\underline{63}, 244-249$

Kirthiga M, Poornima P, Praveen R, Sakeena B, Disha P (2015): Dental erosion and its associated factors in 11-16-year old school children. J Clin Pediatr Dent $\underline{39}, 336-342$

Kitasako Y, Sasaki Y, Takagaki T, Sadr A, Tagami J (2015): Age-specific prevalence of erosive tooth wear by acidic diet and gastroesophageal reflux in Japan. J Dent $\underline{43}, 418-423$

Kumar S, Acharya S, Mishra P, Debnath N, Vasthare (2013): Prevalence and risk factors for dental erosion among 11- to 14-year-old school children in South India. J Oral Sci 55, 329-336

Lacasse A, Rey E, Ferreira E, Morin C, Bérard A (2008): Nausea and vomiting of pregnancy: what about quality of life? BJOG $\underline{115}, 1484-1493$

Landis JR, Koch GG (1977): The measurement of observer agreement for categorial data. Biometrics $\underline{33}, 159-177$

Larsen MJ, Fejerskov O (1989): Chemical and structural challenges in remineralization of dental enamel lesions. Scand J Dent Res 97, 285-296

Larsen MJ, Nyvad B (1999): Enamel erosion by some soft drinks and orange juices relative to their $\mathrm{pH}$, buffering effect and contents of calcium phosphate. Caries Res $\underline{33}, 81-87$ 
Larsen MJ, Poulsen S, Hansen I (2005): Erosion of the teeth: prevalence and distribution in a group of danish school children. Eur J Paediatr Dent $\underline{6}, 44-47$

Lewis KJ, Smith BGN (1973): The relationship of erosion and attrition in extensive tooth tissue loss. Br Dent J 135, 400-404

Lilenfeld LR, Stein D, Bulik CM, Strober M, Plotnikov K, Pollice C, Rao R, Merikangas KR, Nagy L, Kaye WH (2000): Personality straits among currently eating disorders, recovered and never ill first-degree female relatives of bulimic and control women. Psychol Med $\underline{30}$, 1399-1410

Linkosalo E, Markkanen H (1985): Dental erosion in relation to lactovegetarian diet. Scand J Dent Res 93, 436-441

Linnett V, Seow WK (2001): Dental erosion in children: a literature review. Pediatr Dent $\underline{23}, 37-43$

Lippert F, Parker DM, Jandt KD (2004): Susceptibility of deciduous and permanent enamel to dieatary acid-induced erosion studied with atomic force microscopy nanoindentation. Eur J Oral Sci 112, 61-66

Luo Y, Zeng XJ, Du MQ, Bedi R (2005): The prevalence of dental erosion in preschool children in China. J Dent $\underline{33}, 115-121$

Lussi A (2006): Erosive tooth wear - a multifactorial condition of growing concern and increasing knowledge. Monogr Oral Sci $\underline{20}, 1-8$

Lussi A, Schaffner M, Hotz P, Suter P (1991): Dental erosion in a population of swiss adults. Community Dent Oral Epidemiol 19, 286-290

Lussi A, Kohler N, Zero D, Schaffner M, Megert B (2000): A comparison of the erosive potential of different beverages in primary and permanent teeth using an in vitro model. Eur J Oral Sci $\underline{108}, 110-114$

Lussi A, Hellwig E, Zero D, Jaeggi T (2006): Erosive tooth wear: diagnosis, risk factors and prevention. Am J Dent $\underline{19}, 319-25$

Lussi A, Schaffner M, Jaeggi T (2007): Dental erosion - diagnosis and prevention in children and adults. Int Dent J $\underline{57}, 385-398$ 
Lussi A, Schlueter N, Rakhmatullina E, Ganss C (2011): Dental erosion - an overview with emphasis on chemical and histopathological aspects. Caries Res 45, $2-12$

Lussi A, von Salis-Marincek M, Ganss C, Hellwig E, Cheaib Z, Jaeggi T (2012): Clinical study monitoring the $\mathrm{pH}$ on tooth surfaces in patients with and without erosions. Caries Res $\underline{46}, 507-512$

Magalhães AC, Rios D, Honório HM, Delbem AC, Buzalaf MA (2009): Effect of $4 \%$ titanium tetrafluoride solution on the erosion of permanent and deciduous human enamel: an in situ / ex vivo study. J Appl Oral Sci 17, 56-60

Mahoney P (2013): Testing functional and morphological interpretations of enamel thickness along the deciduous tooth row in human children. Am J Phys Anthropol 151, 518-525

Manaf ZA, Lee MT, Ali NH, Samynathan S, Jie YP, Ismail NH, Bibiana Hui Ying Y, Wei Seng Y, Yahya NA (2012): Relationship between food habits and tooth erosion occurrence in malaysian university students. Malays J Med Sci $\underline{19}$, 5666

Mangueira DF, Sampaio FC, Oliveira AF (2009): Association between socioeconomic factors and dental erosion in brazilian schoolchildren. J Public Health Dent $\underline{69}, 254-259$

Mantonanaki M, Koletsi-Kounari H, Mamai-Homata E, Papaioannou W (2013): Dental erosion prevalence and associated risk indicators among preschool children in Athens, Greece. Clin Oral Investig 17, 585-593

Max Rubner-Institut: Nationale Verzehrsstudie II; Ergebnisbericht Teil 2. Bundesforschungsinstitut für Ernährung und Lebensmittel, 2008

McDerra EJ, Pollard MA Curzon ME (1998): The dental status of asthmatic british school children. Paediatr Dent 20, 281-287

Meurman JH, Frank RM (1991): Progression and surface ultrastructure of in vitro caused erosive lesions in human and bovine enamel. Caries Res $\underline{25}, 81-87$ Meurman JH, ten Cate JM (1996): Pathogenesis and modyifying factors of dental erosion. Eur J Oral Sci 104, 199-206 
Millward A, Shaw L, Smith AJ (1994): Dental erosion in four-year-old children in differing socioeconomic backgrounds. J Dent Child $\underline{61}$, 263-266

Millward A, Shaw L, Harrington E, Smith AJ (1997): Continuous monitoring of salivary flow rate and $\mathrm{pH}$ at the surface of the dentition following consumption of acidic beverages. Caries Res $\underline{31}$, 44-49

Moimaz SA, Araújo PC, Chiba FY, Garbín CA, Saliba NA (2013): Prevalence of deciduous tooth erosion in childhood. Int J Dent Hyg 11, 226-230

Mulic A, Skudutyte-Rysstad R, Tveit AB, Skaare AB (2012): Risk indicators for dental erosive wear among 18-year-old subjects in Oslo, Norway: Eur J Oral Sci $\underline{20}, 531-538$

Muller-Bolla M, Courson F, Smail-Faugeron V, Bernardin T, Lupi-Pégurier L (2015): Dental erosion in french adolescents. BMC Oral Health $\underline{15}, 147$

Murakami C, Oliveira LB, Sheiham A, Nahás Pires Corrêa MS, Haddad AE, Bönecker M (2011): Risk indicators for erosive tooth wear in brazilian preschool children. Caries Res $\underline{45}, 121-129$

Nahás Pires Corrêa MS, Nahás Pires Corrêa, F, Nahás Pires Corrêa JP, Murakami C, Mendes FM (2011): Prevalence and associated factors of dental erosion in children and adolescents of a private dental practice. Int J Paediatr Dent $\underline{21}, 451-458$

Naumann CR, Zelig C, Napolitano PG, Ko CW (2012): Nausea, vomiting, and heartburn in pregnancy: a prospective look at risk, treatment, and outcome. $\mathrm{J}$ Matern Fetal Neonatal Med 25, 1488-1493

Nayak SS, Ashokkumar BR, Ankola AV, Hebbal MI (2012): Association of erosion with dietary factors among 5-year-old children in India. J Dent Child $\underline{79}$, 122-129

Nunn JH, Gordon PJ, Morris AJ, Pine CM, Walker A (2003): Dental erosion changing prevalence? A review of british national children's survey. Int J Paediatr Dent $\underline{13}, 98-105$

Ohrn R, Angmar-Mansson B (2000): Oral status of 35 subjects with eating disorders: a 1-year study. Eur J Oral Sci 108, 275-280 
O'Sullivan EA (2000): A new index for the measurement of erosion in children. Eur J Paediatr Dent 1 , 69-74

Peres, KG, Armenio MF, Peres MA, Traebert J, De Lacerda JT (2005): Dental erosion in 12-year-old schoolchildren: a cross-sectional study in Southern Brazil. Int J Paediatr Dent $\underline{15}$, 249-255

Picos AM, Poenar S, Opris A, Chira A, Bud M, Berar A, Picos A, Dumitrascu DL (2013): Prevalence of dental erosions in GERD: a pilot study. Clujul Med $\underline{86}$, $344-446$

Pontefract H, Hughes J, Kemp K, Yates R, Newcombe RG, Addy M (2001): The erosive effect of some mouthrinses on enamel: a study in situ. J Clin Periodontol $28,319-324$

Putz B, Attin T (2002): Die Prävalenz von Erosionen. Dtsch Zahnärztl Z $\underline{57}$, 637343

Robb ND, Smith BG (1990): Prevalence of pathological tooth wear in patients with chronic alcoholism. Br Dent J $\underline{169}$, 367-369

Rytömaa I, Meurman J, Koskinen J (1988): In vitro erosion of bovine enamel caused by acidic drinks and other food stuffs. Scan J Dent Res $\underline{96}, 324-33$

Rytömaa I, Meurman JH, Franssila S, Torkko H (1989): Oral hygiene products may cause dental erosion. Proc Finn Dent Soc $\underline{85}$, 161-166

Rytömaa I, Järvinen V, Kanerva R, Heinonen P (1998): Bulimia and tooth erosion. Acta Odontol Scand $\underline{56}, 36-40$

Salas MMS, Nascimento GG, Vargas-Ferreira F, Tarquinio SBC, Huysmans MCDNJM, Demarco FF (2015): Diet influenced tooth erosion prevalence in children and adolescents: Results of a meta-analysis and meta-regression. J Dent $\underline{43}, 865-875$

Sandström A, Cressey J, Stecksén-Blicks C (2011): Tooth-brushing behavior in 6-12-year olds. Int J Paediatr Dent 21, 43-49

Scheutzel P (1996): Etiology of dental erosion - intrinsic factors. Eur J Oral Sci $\underline{104}, 178-190$ 
Schiffner U, Micheelis W, Reich E (2002): Erosionen und keilförmige Zahnhalsdefekte bei deutschen Erwachsenen und Senioren. Dtsch Zahnärztl Z $\underline{57}$, 102106

Schlueter N, Hardt M, Klimek J, Ganss C (2010): Influence of the digestive enzymes trypsin and pepsin in vitro on the progression of erosion in dentine. Arch Oral Biol $\underline{55}, 294-299$

Schroeder HE: Orale Strukturbiologie. Entwicklungsgeschichte, Struktur und Funktion normaler Hart- und Weichgewebe der Mundhöhle und des Kiefergelenks. 5. Unveränderte Auflage; Thieme, Stuttgart 2000

Scully C (2003): Drug effects on salivary glands: dry mouth. Oral Dis $\underline{9}, 165-176$ Shaw L, O'Sullivan E (2000): UK national clinical guidelines in paediatric dentistry. Diagnosis and prevention of dental erosion in children. Int J Paediatr Dent 10, 356-365

Shellis RP (1984): Variations in growth of the enamel crown in human teeth and a possible relationship between growth and enamel structure. Arch Oral Biol $\underline{29}$, 697-705

Shellis RP, Featherstone JDB, Lussi A: Understanding the chemistry of dental erosion. In: Lussi A, Ganss C (eds): Erosive tooth wear. Monogr Oral Sci. Karger, Basel 2014; 25: 163-179

Smith BG, Knight JK (1984): An index for measuring the wear of teeth. Br Dent J 156, 435-438

Smith BG, Robb ND (1996): The prevalence of toothwear in 1007 dental patients. J Oral Rehabil 23, 232-239

Sognnaes RF, Wolcott RB, Xhonga FA (1972): Dental erosion. I. Erosion-like patterns occurring in association with other dental conditions. J Am Dent Assoc $\underline{84}, 571-576$

Stack MV (1953): Variation in the organic content of deciduous enamel and dentine. Biochem J $\underline{54}$, xv 
Stephan RM (1966): Effect of different types of human foods on dental health in experimental animals. J Dent Res $\underline{45}, 1551-1561$

Stephen KW, McCrossan J, Mackenzie D, Macfarlane CB, Speirs CF (1980): Factors determining the passage of drugs from blood into saliva. $\mathrm{Br} \mathrm{J}$ Clin Pharmacol $\underline{9}, 51-55$

Sullivan RE, Kramer WS (1983): Introgenic erosion of teeth. J Dent Child $\underline{50}$, 192-196

Taji SS, Seow WK, Townsend GC, Holcombe T (2010): A controlled study of dental erosion in 2- to 4-year-old twins. Int J Paediatr Dent 20, 400-409

Tao DY, Hao G, Lu HX, Tian Y, Feng XP (2015): Dental erosion among children aged 3-6 years and its associated indicators. J Public Health Dent $\underline{75}$, 291-297

ten Cate JM, Imfeld T (1996): Dental erosion, summary. Eur J Oral Sci 104, 241-244

Thylstrup A, Fejerskov O: Textbook of cariology. 1. Auflage; Munksgaard, Kopenhagen 1986

Thylstrup A, Fejerskov O: Textbook of clinical cariology. 2. Auflage; Munksgaard, Kopenhagen 1996

Van Rijkom HM, Truin GJ, Frencken JE, König KG, Van't Hof MA, Bronkhorst EM, Roeters FJ (2002): Prevalence, distribution and background variables of smooth-bordered tooth wear in teenagers in The Hague, The Netherlands. Caries $\operatorname{Res} \underline{36}, 147-154$

Vered Y, Lussi A, Zini A, Gleitman J, Sgan-Cohen HD (2014): Dental erosive wear assessment among adolescents and adults utilizing the basic erosive wear examination (BEWE) scoring system. Clin Oral Investig 18, 1985-1990

Vieira A, Overweg E, Ruben JL, Huysmans MC (2006): Toothbrush abrasion, simulated tongue friction and attrition of eroded bovine enamel in vitro. J Dent 34, 336-342 
Wiegand A, Müller J, Werner C, Attin T (2006): Prevalence of erosive tooth wear and associated risk factors in 2-7-year-old german kindergarten children. Oral Dis $\underline{12}, 117-124$

Wiegand A, Köwing L, Attin T (2007): Impact of brushing force on abrasion of acid-softened and sound enamel. Arch Oral Biol $\underline{52}, 1043-1047$

Wiegand A, Bliggenstorfer S, Magalhães AC, Sener B, Attin T (2008): Impact of the in situ formed salivary pellicle on enamel and dentine erosion induced by different acids. Acta Odontol Cand $\underline{66}, 225-230$

Wilson PR, Beynon AD (1989): Mineralization differences between human deciduous and permanent enamel measured by quantitative microradiography. Arch Oral Biol $\underline{34}, 85-88$

Wynn RL, Meiller TF (2001): Drugs and dry mouth. Gen Dent $\underline{49}$, 10-14

Xhonga FA, Valdmanis S (1983): Geographic comparisons of the incidence of dental erosion: a two centre study. J Oral Rehabil 10, 269- 277

Young WG, Khan F (2002): Sites of dental erosion are saliva-dependent. J Oral Rehabil $\underline{29}$, 35-43

Zebrauskas A, Birskute R, Maciulskiene V (2014): Prevalence of dental erosion among the young regular swimmers in Kaunas, Lithuania. J Oral Maxillofac Res $\underline{5}, 2$

Zero DT (1996): Etiology of dental erosion - extrinsic factors. Eur J Oral Sci 104, 162-177

Zero DT, Lussi A: Etiology of enamel erosion - intrinsic and extrinsic factors; In: Addy M, Embergy G, Edgar, WM, Orchardson R (Hrsg.): Tooth wear and sensitivity. Martin Dunitz, London 2000, 121-139

Zhang S, Chau AM, Lo EC, Chu CH (2014): Dental caries and erosion status of 12-year-old Hong Kong children. BMC Public Health 14, 7

Zipkin I, McClure FJ (1949): Salivary citrate and dental erosion, procedure for determining citric acid in saliva - dental erosion and citric acid in saliva. J Dent Res $\underline{28}, 613-626$ 
Danksagung

Ich möchte mich besonders bei Frau Prof. Dr. Annette Wiegand, der Direktorin der Poliklinik für Präventive Zahnmedizin, Parodontologie und Kariologie im Zentrum Zahn-, Mund- und Kieferheilkunde der Medizinischen Fakultät der Universität Göttingen, für die freundliche Überlassung des Themas und die engagierte Unterstützung und Förderung dieser Arbeit bedanken.

Ebenfalls möchte ich mich bei der Deutschen Gesellschaft für Präventivzahnmedizin für die großzügige Förderung dieser Arbeit durch den DGPZM - CP GABA Wissenschaftsfond bedanken.

Ein weiterer Dank gilt Herrn PD Dr. Klaus Jung für die statistische Beratung in der zentralen Serviceeinheit Medizinische Biometrie und Statistische Bioinformatik am Institut für Medizinische Statistik der Georg-August-Universität Göttingen.

Ich möchte mich sehr herzlich bei meinem Ehemann Dr. Stephan Müller für die tatkräftige Unterstützung bei der Durchführung der Untersuchungen in den Kindergärten und die Anregungen bei der statistischen Auswertung bedanken.

Bei Frau Dr. Claudia Tschammler aus der Poliklinik für Präventive Zahnmedizin, Parodontologie und Kariologie möchte ich mich für die kollegiale Zusammenarbeit und die Anregungen bedanken.

Ein großes Dankeschön gilt allen teilnehmenden Kindern und Kindergärten für die freundliche und entgegenkommende Kooperation.

Einen weiteren Dank möchte ich Herrn Matthias Hentschel von der Firma Hentschel-Dental aus Teningen-Nimburg für die großzügige Bereitstellung der Kinderzahnbürsten aussprechen. Die Kinder haben sich sehr über diese Präsente gefreut. 